\title{
PIEZOELECTRIC TRANSDUCER DESIGN OPTIMIZATION
}

by

JUAN MANUEL MELCHOR RODRÍGUEZ

A thesis submitted to the Department of Structural Mechanics and Hydraulic Engineering,

in partial fulfillment of the requirements for the degree of

\section{MÁSTER OFICIAL DE ESTRUCTURAS}

Supervisor: Dr. Guillermo Rus Carlborg

Department of Structural Mechanics and Hydraulic Engineering

University of Granada, Campus de Fuentenueva,

18071 Granada, Spain

September 2012 


\section{ABSTRACT \\ PIEZOELECTRIC TRANSDUCER DESIGN OPTIMIZATION}

To respond to the design of a torsion sensor into mechanical ultrasonic tissue applications, it is necessary to use FEM Finite Element Models. Through a simplified analytical model of torsion transducer, we determine the resonant frequency for a torque transducer ultrasonic waves. It is computationally validated. More specifically the idea is to refine and optimize the design to be applied to the detection of preterm birth identifying changes in the consistency of the cervical tissue through the shear modulus measurements. Therefore, a model with a disk transmitter and a ring receiver for easy accessibility was performed and sensitivity analysis to find the range of optimal design values with this application was calculated. Therefore, it is necessary to optimize the piezoelectric transducer model design regarding two types of parameters. On one hand the design parameters, and on the other hand the model parameters that characterize the specimen. The forward problem is obtained by performing a three-dimensional finite element simulation. The experimental measurements are simulated by adding a gaussian noise as a percentage of the RMS of the numerically predicted signals. In addition, a semi-analytical estimate of the probability of detection (POD) is developed to provide a rational criterion to optimize the experimental design. 


\section{RESUMEN}

\section{OPTIMIZACIÓN DEL DISEÑO DE UN TRANSDUCTOR PIEZOELÉCTRICO}

Para responder al diseño de un sensor de torsión con aplicaciones a la mecánica tisular ultrasónica, es necesario el uso de modelos de elementos finitos FEM como procedimiento directo. A través de un modelo simplificado de análisis de transductor de torsión, se determina la frecuencia de resonancia que se valida computacionalmente. Más específicamente, la idea es refinar y optimizar el diseño que debe aplicarse a la detección de parto prematuro identificar los cambios en la consistencia del tejido del cuello uterino a través de medidas del módulo G . Por lo tanto, se elige un modelo con un disco transmisor y un anillo receptor para facilitar la accesibilidad en el diseño y se realizó un análisis de sensibilidad para encontrar el rango de valores óptimos con esta aplicación. Para optimizar el diseño del modelo del transductor piezoeléctrico con respecto a dos tipos de parámetros. Por un lado los parámetros de diseño, y por otra parte los parámetros del modelo que caracterizan la muestra. Las mediciones experimentales se simulan mediante la adición de un ruido gaussiano como un porcentaje de la RMS de las señales predichas numéricamente. Además, una estimación semi-analítica de la probabilidad de detección (POD) se ha desarrollado para proporcionar un criterio racional para optimizar el diseño experimental. 


\section{ACKNOWLEDGMENTS}

I would like to begin by thanking this work Guillermo Rus Carlborg for their effort and dedication throughout the process. Achieved convey enthusiasm for research and especially in the exciting area of Ultrasonic Tissue Mechanics. Your support and friendship has managed to motivate learning throughout this period.

I would also like to thank my colleagues of the Non Destructive Evaluation Laboratory, Nicolas, Juan, Manuel, Laura, Roberto and Paloma for giving me such good advice, and share great moments in and out of the research work. I couldn't forget my colleges of the Department of Structural Mechanics I would like to thank for their friendly help and advise.

Finally, I would like to dedicate this work to Mia, Caroline, Juan and Nati and express my sincere gratitude for your constant understanding, support and love in my career.

This work has been supported by the Ministry of Education of Spain through Dpi2010-17065. 


\section{AUTORIZACIÓN}

D.

Profesor del departamento de

de la Universidad de Granada, como director del Proyecto Fin de Máster de D.

Informa:

que el presente trabajo, titulado:

Ha sido realizado y redactado por el mencionado alumno bajo nuetra dirección, y con esta fecha autorizo a su presentación.

Granada, a ..... de de $20 \ldots .$.

Fdo. 
Los abajo firmantes autorizan a que la presente copia de Proyecto Fin de Máster se ubique en la Biblioteca del Centro y/o departamento para ser libremente consultada por las personas que lo deseen.

Granada, a ..... de ............ de $20 \ldots .$.

(Firmas y números de DNI / NIE del alumno y de los tutores) 


\section{Informe de valoración del proyecto}

El tribunal constituido para la evaluación del Proyecto Fin de Máster titulado:

Realizado por el alumno:

Y dirigido por el tutor:

Ha resuelto la calificación de:

SOBRESALIENTE (9-10 puntos)

NOTABLE (7-8.9 puntos)

APROBADO (5-6.9 puntos)

\section{SUSPENSO}

Con la nota ${ }^{1}$ : puntos.

El Presidente:

El Secretario:

El Vocal:

Granada, a ..... de de $20 \ldots .$.

\footnotetext{
${ }^{1}$ Solamente con un decimal.
} 


\section{Contents}

Resumen $\quad$ iii

$\begin{array}{ll}\text { Abstract } & 1\end{array}$

1 Introduction $\quad 1$

1.1 Motivation and Objectives . . . . . . . . . . . . 1

1.2 Thesis Organization . . . . . . . . . . . . . . 7

2 FEM: Finite element model and sensitivity analysis for tor$\begin{array}{ll}\text { sion transducer } & 8\end{array}$

2.1 Introduction . . . . . . . . . . . . . . . . . 8

2.2 Finite Element Analysis . . . . . . . . . . . . . . . . . . 9

2.2.1 Piezoelectric FEM . . . . . . . . . . . . . . . . . . . 10

2.2.2 Model A description . . . . . . . . . . . . . . . . 10

2.2.3 Model B description . . . . . . . . . . . . . . 15

2.2.4 Parametric model . . . . . . . . . . . . . . 15

2.3 Simplified model of torsion transducer . . . . . . . . . . . 16

2.3.1 Assumptions . . . . . . . . . . . . . . . . . . 17

2.3.2 Subsystem eigenfrequency in the case of cylinder mass 19

2.3.3 Subsystem eigenfrequency in the case of ring mass . . 19

2.3.4 Validation . . . . . . . . . . . . . . 20

2.4 Convergence . . . . . . . . . . . . . . . . . . 20

2.5 Sensitivity Analysis . . . . . . . . . . . . . . 22

2.5.1 Sensitivity Sensor Dimensions Analysis . . . . . . . . 22

2.5.2 Sensitivity Tissue Analysis . . . . . . . . . . . . . 28

2.6 Conclusions . . . . . . . . . . . . . . . . 33

3 POD: Probability Of Detection of Pathologies in soft tissue 40

3.1 Introduction . . . . . . . . . . . . . . . . 42

3.2 Inverse Procedure . . . . . . . . . . . . . . . . . . 43

3.2.1 Cost functional . . . . . . . . . . . . . . . 44

3.2.2 Probability of detection . . . . . . . . . 45

3.3 Numerical Results . . . . . . . . . . . . . . . . . . . . . . . . 49

3.3.1 Excitation, model displacement . . . . . . . . . . . 49

3.3.2 Effects on cost functional . . . . . . . . . . . 50

3.3.3 Effects on POD . . . . . . . . . . . . 50

3.3.4 POD optimization . . . . . . . . . . . 50 
3.4 Conclusions and impact . . . . . . . . . . . . . 52

3.4.1 Conclusions .................... 52

3.4 .2 Impact . . . . . . . . . . . . . . . 53

A 3D piezoelectric laminate validation $\quad 56$

A.1 Analytic 1D validation . . . . . . . . . . . . . 56

A.2 Results ...................... 63

B Torsion sensor model algorithms $\quad 66$

$\begin{array}{lr}\text { List of Figures } & 89\end{array}$

$\begin{array}{ll}\text { List of Tables } & 94\end{array}$

$\begin{array}{lr}\text { Bibliography } & 96\end{array}$ 


\section{Chapter 1}

\section{Introduction}

\subsection{Motivation and Objectives}

Piezoelectric ceramic materials, such as lead zirconate titanate (PZT) are now widely used in solid-state actuators and sensors which were designed for numerous applications, such as precision positioning, noise and vibration sensing and cancellation, linear motors, and ultrasound techniques in our case. This work focuses on designing and optimizing a transducer capable of transmitting and receiving torsional ultrasound waves. In many of those applications, a large torsional displacement is required, for example, in robotics to achieve the micropositioning, in CD drivers, in helicopters to control the trailing edge flaps (TEF) of rotor blades, etc. Otherwise torsional actuators are capable of producing both large torque and large angular displacement in a compact package, sufficient to meet many smart structures requirements, and can be tailored for a variety of application re-

quirements [1]. Longitudinal-torsional coupling have been explored, such as exponential decay horns and layering of different materials, but in our case is an effect to minimize [2].

There are several optimization criteria of ultrasonic sensors, where the 
conventional characteristics of performance have been being replaced with time. For example, the use of center frequency (maximum frequency in the amplitude spectrum) and the round trip insertion $\operatorname{loss}^{1}$ (which is a parameter associated with the frequencies that fall in the passband of a filter) are not the most appropriate when applied to models of multi spectrum. The new parameters such as centroid frequency and energy factor round, has showed they are more suitable. Other new features describing the waveform and amplitude spectra have been introduced to improve the input parameters of the optimization algorithm, which facilitates the extension for different applications as is the case in this study. The great advantage of doing a design and manufacturing it is that the overall performance we get is explicit and quantitative but also takes into account the point of view of application to which it is addressed, thus avoiding the ambiguous response that can find customer [4].

The combined use of finite element simulation and optimization methods involves a convenient way to design ultrasonic transducers. When using the finite element analysis (FEA) based on the optimization of transducers requires a global cost function as a criteria to minimize, that depends on the model evaluation. The evaluation of cost functions for different characteristics of the transducers, and comparison of results for the different simulations and the resulting sound field carry different design alternatives [5], [6]. The use of finite element analysis combined with the multi-objective decision makes it possible to produce a good design of ultrasonic sensor in

\footnotetext{
${ }^{1}$ One measure of overall round-trip efficiency is insertion loss. Efficiency is measured by comparing the power load resistor $R_{f}$ with the transducer in place to the power there without the transducer. Insertion loss is defined as the ratio of the power in $R_{f}$ over that available from the source generator. $I L(f)=\frac{W_{f}}{W_{g}}=\left[\left|\frac{V_{f}}{V_{g}}\right|^{2}\left(\frac{R_{f}+R_{g}}{R_{f}}\right)\right]$ and in $\mathrm{dB}$, it is $I L_{d B}(f)=10 \log _{10} I L(f)$. Where $W_{f}$ is the power in $R_{f}$ and $V_{g}$ is the source.[3]
} 
tip shape and operating frequency, where the conflict of multiple criteria concerning vibration, impedance and acoustic effects of transducer are optimized while the design time and is very satisfactory for decisions of the designer. As a result, significantly improves the standard values of all the criteria considered [7]. Transfer matrix methods the design of a Langevin transducer has been formulated as a constrained multiobjective optimization problem involving continuous and discrete design variables [8].

The piezoelectric transducers convert electrical signals into mechanical vibrations and vice versa. Bolt-clamped Langevin Type (BLT), consist of piezoelectric disks and two elastic blocks fitted at each end, efficiently generating mechanical vibrations of the desired frequencies. They generally be applied as actuators in most applications. Most of them use longitudinal vibration in the thickness direction of the piezoelectric disk. However there are studies where torsional vibrations generated by elastic rod working as a viscous sensor. From the analytical point of view the shear BLT studied by deriving the equations features and modes of vibration [9],[10]. The longitudinal bending mode is composed of a symmetrical structure, the piezoelectric ceramic elements are located near the geometric center of the transducer [11],[12]. At first they were designed to generate uniform droplets micro [13], but are also used in the near field acoustic levitation (NFAL) which caused the coupling of the longitudinal mode of the Langevin type transducer with the flexural mode of the top plate used in the areas of nondestructive evaluation, underwater acoustics and bioengineering [14]. And there is also the case of micro torsional mirrors, optical scanners used as in high frequency. The micro torsional mirror consists of a driving mechanism and a reinforced mirror plate. And have four advantages fundamentally the driving voltage is lower due to the use of the gap-closing parallel-plate 
electrodes, and the traveling distance is remarkably magnified by the lever mechanism; the mirror plate is subjected to a pure torque generated by the driving mechanism, thereby preventing wobble motion of the mirror plate; the stiffness and flatness of the mirror plate is increased by incorporating a reinforced frame, which improves the optical performance of the scanner; and the mirror can be used at a higher scanning frequency due to the higher vibration mode of the three-mass system [15].

The numerical approaches used can be used to evaluate other types of transducer designs and to establish tolerance limits for each of the design parameters required to maintain transducer performance within a specified range [16]. Also, the differential equations of piezoelectric torsional motions are derived in terms of the circumferential displacement and the electric potential [9]. The analysis shows that compliance effects increase with applied shear rate and that the effects of torsional and axial compliance are coupled in measurements of the shear stress and first normal stress difference [17]. The analytical models of the transducers system are established on the basis of electromechanical equivalent circuitry theory, vibration theory and wave theory, which lay the foundation for determining the initial topological information of the ultrasonic transducer. The resonance frequency, vibration displacement nodes and rule of ultrasonic energy transmission are acquired by making modal and harmonic analysis [18].

The application of ultrasonic sensors in the clinical field, is covered mainly by the ultrasound waves through the treatment of $\mathrm{P}$ type, so that there is a high interest in the design of optimized efficiency transducers. A specific contribution is to reduce the pulse length acoustic transmitter transducer, this improves the bandwidth of the transducer and thus reduces their 
factor Q (quality factor or selectivity factor is a parameter which measures the ratio between the energy reactive stored and dissipated during a complete cycle of the signal). Another optimization can be performed in the dynamic range of the transducer, which is of great importance because of the extremely large differences in reflectance that occurs in the human body [19].

Moreover, the intensity of $100 \mathrm{~mW} / \mathrm{cm}^{2}$ is considered clinically safe even for prolonged exposure, and is intended to clinically relevant therapeutic applications, including treatment of wounds and transdermal drug delivery. Being able to produce this level of intensity with only $15-20 \mathrm{~V}$ offers a complete wireless portable use attractive and both patients and doctors, and potentially increasing patient compliance with doctors recommendations [20].

It is worth mentioning the case of devices designed to measure the stiffness in the regeneration of distraction osteogenesis, although it is not an ultrasonic sensor, torsional rigidity is evaluated by applying a torque minimum. This method exhibits strong predictive power for the torsional rigidity in bone fractures, and has great potential for monitoring of fracture healing $[21]$.

The aim of this work is to design an ultrasonic transducer for clinical applications in the field of tissue characterization with high sensitivity, which can be defined and made now or in the near future. Since the design for the frequency dependent attenuation in biological tissues is an important topic for research in this field. 
We propose a feasible torque transducer to record information on the mechanical shear modulus in tissues, then it was simulated under a FEM (Finite Element Model) in FEAP software, revealing the main mechanisms. Subsequently confirmed with an analytical model to find the main correlations between the parameters of the numerical model.

The results obtained after a parametric sensitivity analysis in both dimensions of the transducer as in the layers of tissue through which the wave propagates relate the possibility of manufacturing the second sensor design optimal. 


\subsection{Thesis Organization}

This thesis is organized as follows: The present chapter deals with the motivation and organization of the research work presented herein. Chapter 2 is dedicated to direct model formulation in finite element models of two possible torsional piezoelectric sensors for medical diagnostic and sensitivity analysis of the best model. It is presented in the format of a scientist paper prepared to be submitted to Special Issue "Piezoelectric Sensors and Actuators" - Sensors (ISSN 1424-8220). Chapter 3 treats the optimization of the model developed in the previous chapter using the inverse problem and the formulation of the POD (Probability of Detection) of pathologies in soft tissue, which is also prepared to be submitted to Ultrasonics-Elselvier (ISSN: 0041-624X). Finally, this document is closed with two appendices: Appendix A develops the formulation of the 3D laminate piezoelectric validation and Appendix B is dedicated to the Torsion Sensor Model algorithms, developed for this research. 


\section{Chapter 2}

\section{FEM: Finite element model and sensitivity analysis for torsion transducer}

\section{$2.1 \quad$ Introduction}

To respond to the design of a torsion sensor into mechanical ultrasonic tissue applications, it is necessary to use FEM Finite Element Models. Using a simplified analytical model of torsion transducer, we determine the resonant frequency for a torque transducer ultrasonic waves. Using FEAP software, we model the sensor consists of materials such as steel, piezoelectric ceramic. In a first model A the notion torque sensor for applications in tissue mechanics is generalized. It is manufactured and validated in tissue consisting mainly collagen. In a second design B, more specifically the idea is to refine and optimize the design to be applied to the detection of preterm birth identifying changes in the consistency of the cervical tissue through the shear modulus measurements. Therefore, a model with a disk transmitter and a ring receiver for easy accessibility was performed and sen- 
sitivity analysis to find the range of optimal values for the design with this application was calculated.

\section{$2.2 \quad$ Finite Element Analysis}

The modeled transducer is a torsion type with PZT-5A ceramics, with a steel top and bottom central discs and external rings respectively. The operating frequency predicted for the transducer, and subsequently verified by experiment, is $28 \mathrm{kHz}$. The piezoelectric properties of PZT-5A are provided by the material library in FEAP, and the Rayleigh stiffness damping is calculated from the appropriate material, as cited in a variety of sources.

Excitation is provided by a boundary condition of $100 \mathrm{~V}$ peak-to peak with spike function based on Heaviside function:

$$
f=A H(t) H(D-t) \frac{t}{D}
$$

where $\mathrm{A}$ is amplitude, $\mathrm{H}$ is Heaviside function, $\mathrm{D}$ is the distance to origin in $\mathrm{x}$ axe and $\mathrm{t}$ is time.

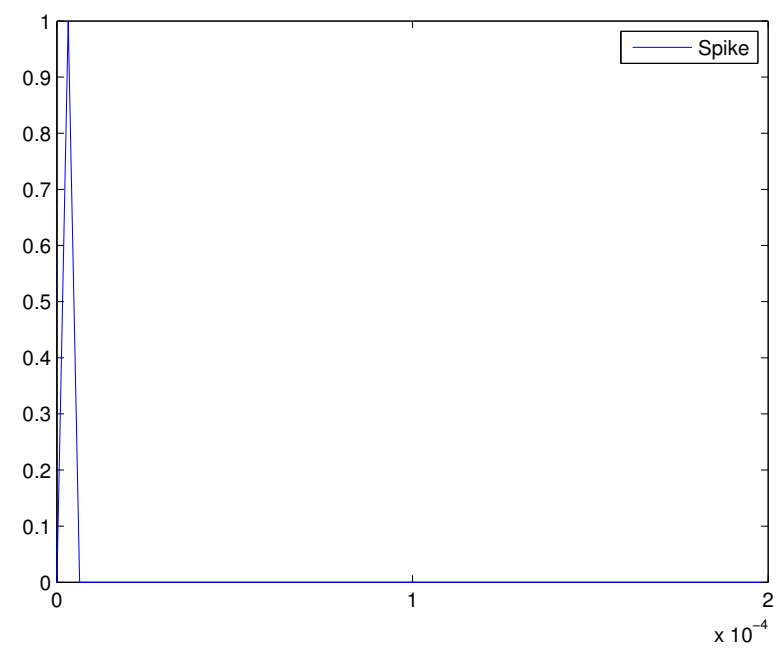

Figure 2.1: Spike function 
Each FE model yields longitudinal (Z) and tangential (X) vibration amplitude data for a point on the edge with reference to the mode shape, these amplitude data can be combined to yield torsionality.[2]

\subsubsection{Piezoelectric FEM}

Piezoelectricity is described analytically within constitutive equation, defined how stress $(\mathrm{T})$, strain $(\mathrm{S})$, charge-density displacement $(\mathrm{D})$, and electric field (E) interact.

Constitutive law (in Strain-Charge form) is:

$$
\begin{aligned}
& S=S_{E} \cdot T+d^{t} \cdot E \\
& D=d \cdot T+\varepsilon_{T} \cdot E
\end{aligned}
$$

Strain-charge to stress-charge transformation have been used to introduce piezoelectric properties according material definition in FEAP (Finite Elements Analysis Program) Software developed by R.Taylor[22]:

$$
\begin{aligned}
C_{E} & =S_{E}^{-1} \\
e & =d \cdot S_{E}^{-1} \\
\varepsilon_{S} & =\varepsilon_{T}-d \cdot S_{E}^{-1} \cdot d^{t}
\end{aligned}
$$

\subsubsection{Model A description}

The numerical tool selected for solving the response of the model (forward problem) is the Finite Element Method (FEM). A 8-node quadratic finite element and 26 blocks, has been implemented. That solves the model given by constitutive equations describe below: 


$$
\begin{aligned}
& S=S_{E} \cdot T+d^{t} \cdot E \\
& D=d \cdot T+\varepsilon_{T} \cdot E
\end{aligned}
$$

where stress $(\mathrm{T})$, strain (S), charge-density displacement (D), and electric field (E)

And the equilibrium and compatibility describe below:

$$
\begin{aligned}
\nabla \cdot D & =0 ; E=-\nabla \phi \\
\nabla^{S} \cdot T & =0 ; S=\frac{1}{2}\left(\nabla u+\nabla u^{t}\right)
\end{aligned}
$$

where $u=(u, w)$ denotes the displacement in directions $\mathrm{x}$ and $\mathrm{z}$, respectively, and $\phi$ is the electric potential or voltage. Finally, the following standard sign criteria is used: the electric field and stress values are considered positive for the direction of polarization of the material and for tractions, respectively.

Piezoelectric element was developed in using the research academic finite element code FEAP [22], and was validated against the analytical solutions obtained for a piezoelectric 3D laminate liable to $100 \mathrm{~V}$ of voltage. [23]

A 3D model of the piezoelectric sensor is simulated by a FEM (Finite Elements Method) using FEAP[24]. 8-noded 3D solid hexaedric elements using a structured mesh are adopted in order to avoid remeshing perturbation on sensitivity analyses. Linear elastic constitutive laws are used with 4 degrees of freedom per node. Anisotropy is considered since the sensors are placed to generate a prevalent transverse wavefront. The model is reduced by considering biaxial symmetry (one quarter of the problem is simulated). The design parameters implied in finite elements model are summarized in table 1 . 


\begin{tabular}{lcc}
\hline \hline Parameter & Value / range & Units \\
\hline Diameter of sensor & 14.224 & {$[\mathrm{~mm}]$} \\
Ceramic thickness & 5.08 & {$[\mathrm{~mm}]$} \\
Piezoelectric material thickness & 0.010 & {$[\mathrm{~mm}]$} \\
Metal thickness & 1 & {$[\mathrm{~mm}]$} \\
Resin thickness & 2 & {$[\mathrm{~mm}]$} \\
Angle of section & $\pi / 4$ & {$[\mathrm{rad}]$} \\
Angle of support to parametrization & asin $(b / R)$ & {$[\mathrm{rad}]$} \\
Total time & 3000 & {$[\mu \mathrm{s}]$} \\
Incremental time & 20 & {$[\mu \mathrm{s}]$} \\
High of piezoelectric material & 10 & {$[\mathrm{~mm}]$} \\
Width of piezoelectric material & 1 & {$[\mathrm{~mm}]$} \\
long of piezoelectric material & 3 & {$[\mathrm{~mm}]$} \\
\hline \hline
\end{tabular}

Table 2.1: Parameters properties implied in sensor mesh analysis.

The model system in which the defect will be sought is defined by the specimen geometry and material, the boundary conditions, the applied displacement, and the detected displacement as output data. The transducer simulated consists of a four material sensor. The mechanical and geometrical material properties of the stratified system are summarized in Table 2.1 and represented in Figure 1.

Lead Zirconate Titanate (PZT-5A) piezoelectric material has been tested in design process characterized by next properties:

- Crystal symmetry class: Uniaxial

- Density: $7750 \mathrm{~kg} / \mathrm{m}^{3}$ 


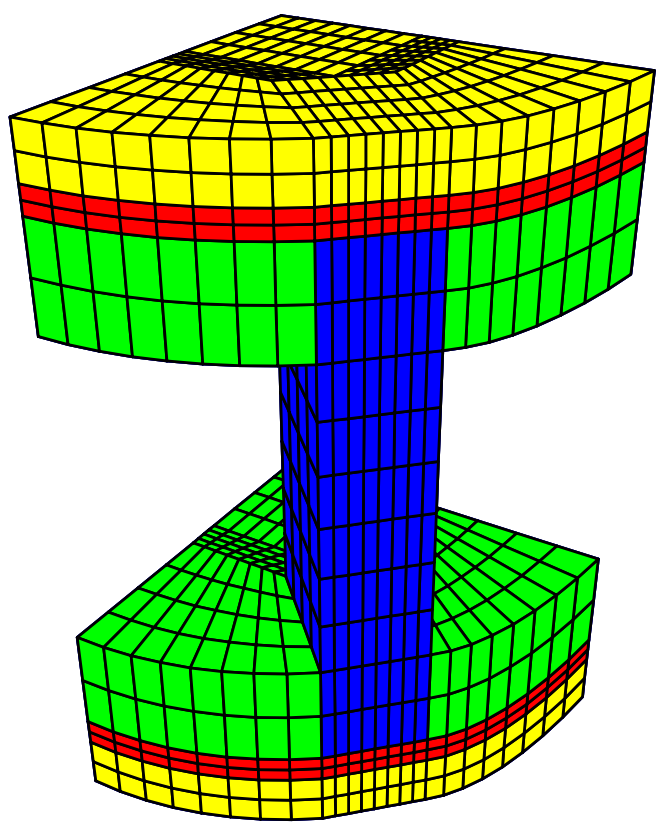

Figure 2.2: FEM for Sensor in Model A

- Compliance:

$$
S_{E}=\left|\begin{array}{cccccc}
16.4 & -5.74 & -7.22 & 0 & 0 & 0 \\
-5.74 & 16.4 & -7.22 & 0 & 0 & 0 \\
-7.22 & -7.22 & 18.8 & 0 & 0 & 0 \\
0 & 0 & 0 & 47.5 & 0 & 0 \\
0 & 0 & 0 & 0 & 47.5 & 0 \\
0 & 0 & 0 & 0 & 0 & 44.3
\end{array}\right| * 10^{-12} \mathrm{~m}^{2} / N
$$

- Piezoelectric Coupling:

$$
d=\left|\begin{array}{cccccc}
0 & 0 & 0 & 0 & 584 & 0 \\
0 & 0 & 0 & 584 & 0 & 0 \\
-171 & -171 & 374 & 0 & 0 & 0
\end{array}\right| * 10^{-12} C / N
$$

- Relative permittivity:

$$
\frac{\varepsilon_{T}}{\varepsilon_{0}}=\left|\begin{array}{ccc}
1730 & 0 & 0 \\
0 & 1730 & 0 \\
0 & 0 & 1730
\end{array}\right|, \varepsilon_{0}=8.854 * 10^{-12} \mathrm{~F} / \mathrm{m}
$$


The system in which the defect will be sought is defined by the geometry, material of the specimen, the boundary conditions, and applied loads and the measuring points as output data.

The transducer consists of a 90 grades sector with four materials piezoelectric, aluminium, ceramic and resin. The PZT-5A laminate is considered as rectangular shaped of size $L x 1[\mathrm{~mm}], L y 1[\mathrm{~mm}]$ and $L z 3[\mathrm{~mm}]$, as shown in Fig. 1. This sample is excited by electrical load, and its response is measured at $N_{i}=4$ points along the lower boundary of the sensor. The selection of electrical load is one of the goals of this study. It should be noted that the electrical loads generate mechanical displacements (inverse effect).

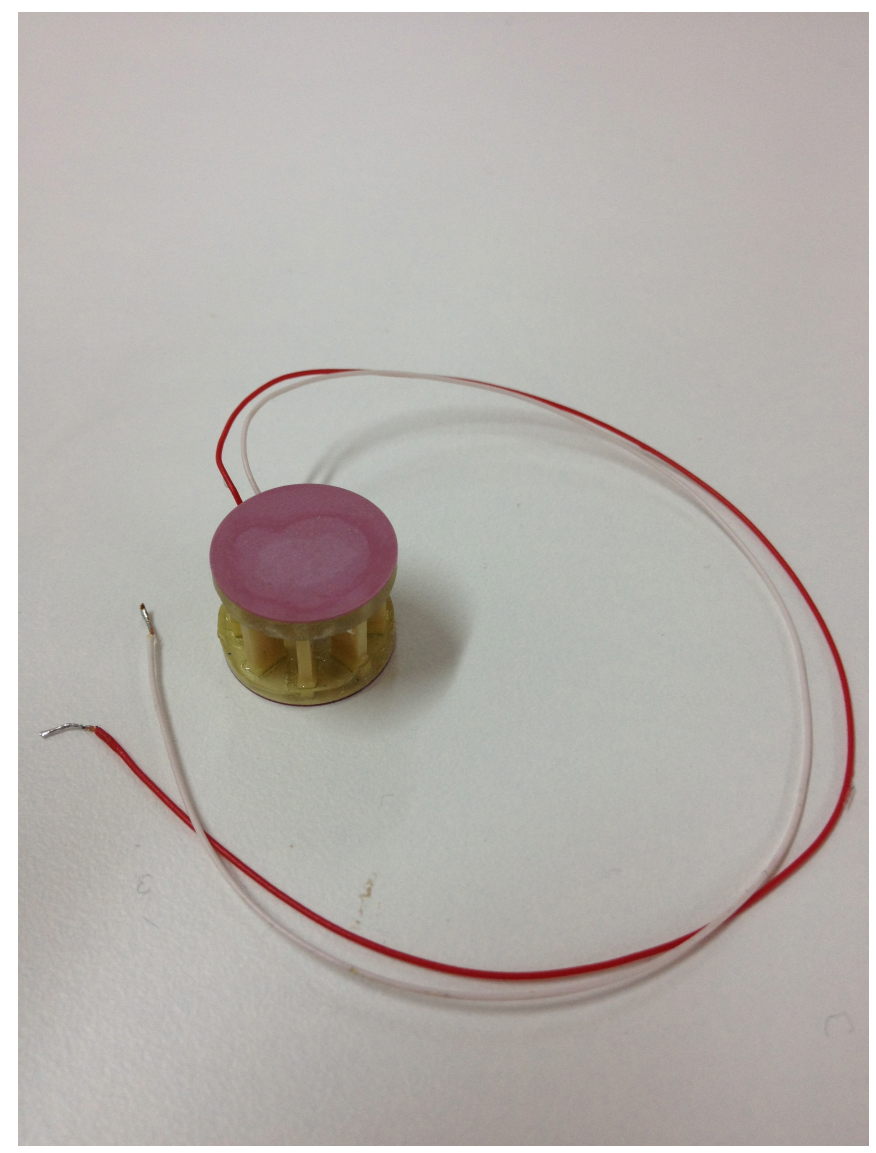

Figure 2.3: Sensor A: Valpey Fischer fabrication 


\subsubsection{Model B description}

Due to the difficulty that may lead to mechanical parameter measurement and detection of soft tissue pathologies with the model A, we decided to develop a design a model $\mathrm{B}$ where the sensor S-wave receiver is a circular crown shape that can be encapsulated in one device.

The geometry is similar to the above but obviously changes the receiving transducer and boundary conditions, as is done by a new finite element calculation (FEM). The new specimen comprises a 90 degree sector of a circular crown 90 degrees. The cyclic boundary conditions ensures that the behavior is appropriate for 360 degrees.

The PZT-5A laminate is considered as rectangular shaped of size $L x 3[\mathrm{~mm}]$, Ly $1[\mathrm{~mm}]$ and $L z 1[\mathrm{~cm}]$ too, as shown in Fig. 2.4.

Parametrization in FEAP consists in 86 blocks within disc transmitter, crown receiver and two layers of dermic and connective tissue respectively.

\subsubsection{Parametric model}

The torque sensor simulation was based on the dimensions of the simplified analytical model, spelled out in the table below, where A, B and L are the width, length and thickness of the sheet of piezoelectric respectively, dd, ds and dr are the diameters of the piezo disk, disk and ring respectively, and $\mathrm{Lz}$ and the song and the thickness of the ring.

The following describes a scheme with the outline of the transmitter and reception consensuses:

The materials were used in the design, steel, aluminum, carbon fiber and ceramic pmma with the following mechanical properties: 


\begin{tabular}{c|cc}
\hline \hline Design Parameters & Optimal dimensions $[\mathrm{mm}]$ & Label \\
\hline Width piezo & 1 & $\mathrm{~A}$ \\
Length piezo & 1 & $\mathrm{~B}$ \\
Thickness piezo & 2 & $\mathrm{~L}$ \\
Inner distance & 5 & $\mathrm{dd}$ \\
Outer radius & 8.5 & $\mathrm{ds}$ \\
Inner radius & 13 & $\mathrm{dR}$ \\
Length outer sensor & 2 & $\mathrm{e}$ \\
Steel thickness & 8 & $\mathrm{Lz}$ \\
\hline \hline
\end{tabular}

Table 2.2: Previous dimensions of sensors

\begin{tabular}{c|ccc}
\hline \hline Material & Young Modulus [MPa] & Poisson coefficient & Density $\left[\mathrm{kg} / \mathrm{m}^{3}\right]$ \\
\hline Steel & 210 & 0.3 & 7800 \\
Aluminium & 65 & 0.3 & 2700 \\
Carbon & 150 & 0.3 & 1500 \\
Pmma & 3 & 0.3 & 1200 \\
Ceramic & 80 & 0.3 & 1800 \\
\hline \hline
\end{tabular}

Table 2.3: Materials used in the design

\subsection{Simplified model of torsion transducer}

A simplified analytical model of the fundamental oscillatory movement of the torsion ultrasonic transducer is derived. To this end, a number of assumptions are carried out mainly on the relevance of elements of the design and on their movement shape (eigenmode), in order to arrive at a single degree of freedom system. Second, the piezoelectric element is assumed to have a predominant deformation law uniformly distributed and linearly proportional to the electrical excitation. 

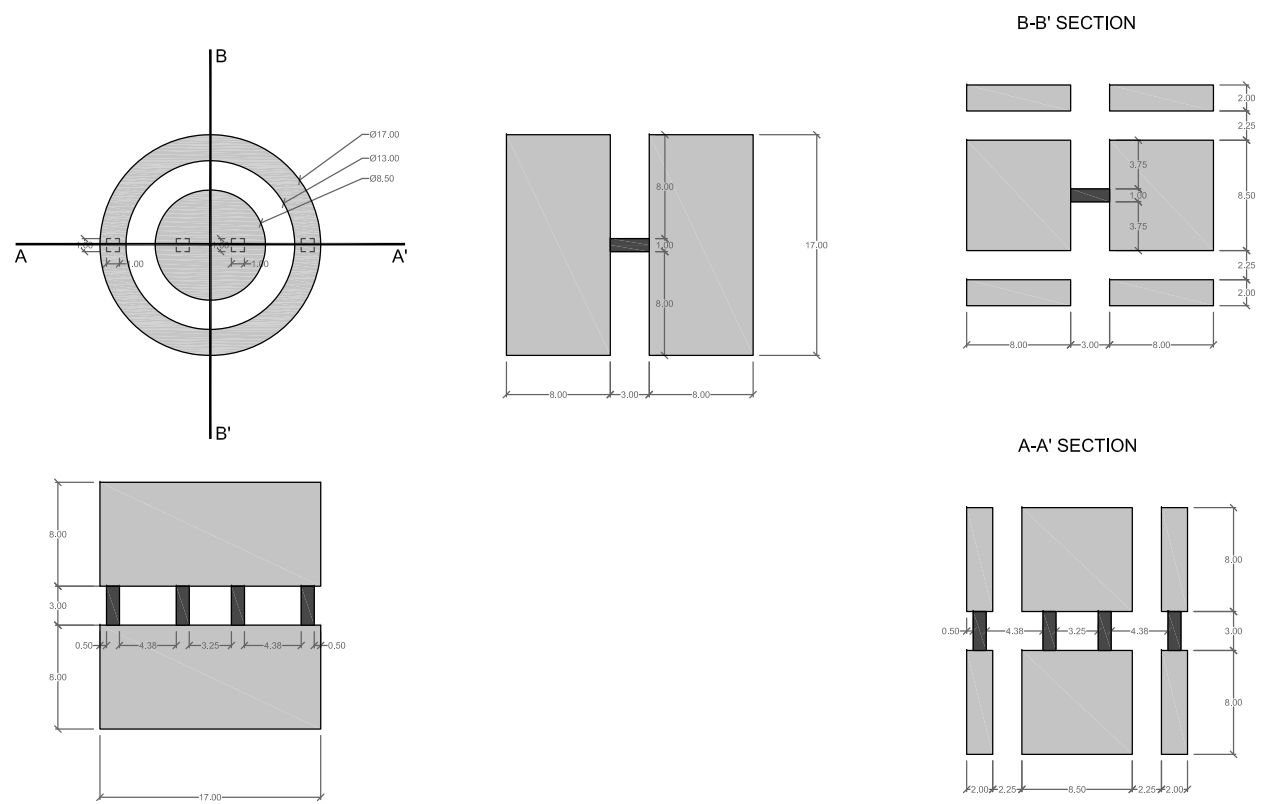

Figure 2.4: The geometry of the transducer

\subsubsection{Assumptions}

1. Reduction to a single-degree-of-freedom system, where the eigenproblem reduces to a single frequency and a single mode (steady-state movement shape).

2. Movement is assumed to be dominated by torsion rotation $\theta$ in radians.

3. Dynamic equilibrium of torsional moment:

$$
k \theta+I \ddot{\theta}=0
$$

where $k$ is the stiffness in $[\mathrm{Nm} / \mathrm{rad}]$ and $I$ is the inertia.

4. Steady-state solution has the form:

$$
\theta=\theta^{0} \sin (\omega t)
$$

as the transient one is neglected for computing the eigenvalue. 
5. Natural frequency (eigenvalue that fulfills the equilibrium 2.14):

$$
\omega=\sqrt{\frac{k}{I}}
$$

in $[\mathrm{rad} / \mathrm{s}]$, or $f=\frac{\omega}{2 \pi}$ in $[\mathrm{Hz}]$.

6. Stiffness of piezoceramic due to moment:

$$
k=\frac{M}{\theta}
$$

where $M$ is the applied torsional moment.

7. Since each of the $n$ piezoceramic elements is dominated by shear deformation, and they are located at distance $d$ from the center of rotation

$$
M=n d F=n d a b \sigma_{x z}
$$

where $F$ is the resulting force of the shear stress $\sigma_{x z}$ applied over the area $a \times b$ of the element.

8. The element described above deforms due to the shear stress the amount $\epsilon_{x z}$, which creates the differential displacement $u=l / \epsilon_{x z}$ between upper and lower sides separated distance $l$. This links to the rotation of the circular array of piezoceramic elements,

$$
\theta=u / d=l / d \epsilon_{x z}
$$

9. The effective length of the piezoelectric elements is reduced as $l^{\text {eff }} \simeq 2 l$ to account for the flexibility of the clamping into the mass. Hence, $\theta=l^{\mathrm{eff}} / d \epsilon_{x z}$.

10. The piezoceramic behaves linearly elastic

$$
\sigma_{x z}=G^{*} \epsilon_{x z}
$$

with modified shear stiffness $G^{*}$ adding piezoelectric coupling [9]. 
11. The inertia against rotation is dominated by the mass blocks of density $\rho$, which are either cylindrical or annular (ring-shaped) of radius $r$. The inertia of the piezoelectric and other elements is comparatively neglectible.

12. Inertia and mass of cylinder:

$$
I=\frac{m r^{2}}{2}, \quad m=\pi r^{2} h \rho
$$

where $h$ is the height of the cylinder (in the axial direction).

13. Inertia and mass of ring:

$$
I=m r^{2}, \quad m=2 \pi r h e \rho
$$

where $e$ is the thickness of the ring (in the radial direction).

\subsubsection{Subsystem eigenfrequency in the case of cylinder mass}

Combining equations 2.14 to 2.21 ,

$$
f=\frac{1}{2 \pi} \sqrt{\frac{n a b d^{2} G}{\frac{\pi}{2} l^{\mathrm{eff}} h r^{4} \rho}}
$$

\subsubsection{Subsystem eigenfrequency in the case of ring mass}

Combining equations 2.14 to 2.22 ,

$$
f=\frac{1}{2 \pi} \sqrt{\frac{n a b d^{2} G}{2 \pi l^{\mathrm{eff}} h e r^{3} \rho}}
$$

When the transducer contains both a cylinder mass and a ring mass for each of the transmitting and receiving subsystems, their eigenfrequencies should be matched in order to maximize the combined resonance amplification. 


\subsubsection{Validation}

Given the frequency of resonance FEM model we proceed to measure the time between peaks of cycles and it is found that coincide with the frequencies of the sensor with the degree of error which is described in the next table.

\begin{tabular}{c|c}
\hline \hline Disc Frequency [Hz] & $2.7977 \mathrm{e}+04$ \\
Ring Frequency $[\mathrm{Hz}]$ & $2.8180 \mathrm{e}+04$ \\
FEM Frequency [Hz] & $2.8169 \mathrm{e}+04$ \\
Error 1 $(\%)$ & 0.6802 \\
Error 2 $(\%)$ & -0.0392 \\
\hline \hline
\end{tabular}

Table 2.4: Validation analytic design vs. FEM

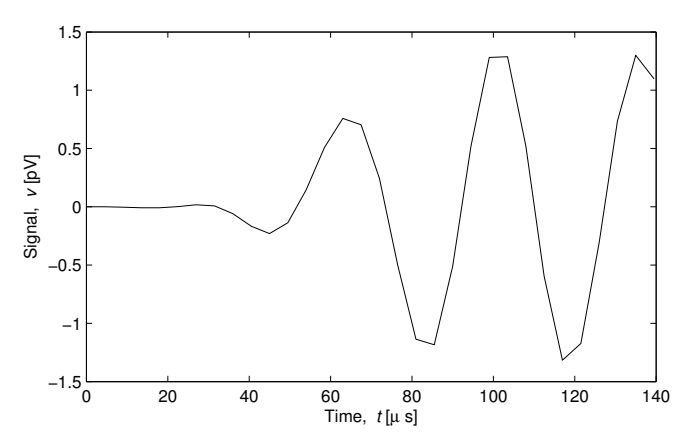

Figure 2.5: example of measurement validating simple design

\subsection{Convergence}

The convergence was based on finding the time increment should vary in a range between $12[n s]$ and $3200[n s]$ obtained as described by the following figures $25[n s]$ as the optimal time step:

The study of convergence of the model geometry was obtained from twelve mesh parameters $q_{3}, n, m, n_{2}, q_{5}, q_{2}, q_{1}, q_{4}, l, p, l_{1}$ and $l_{2}$ and once 

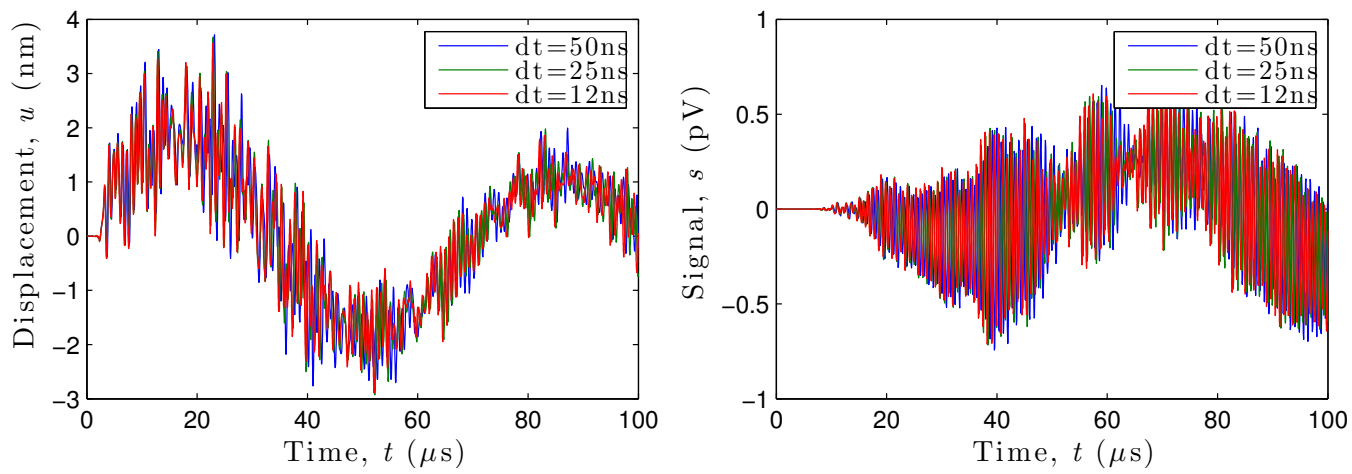

Figure 2.6: Time step convergence
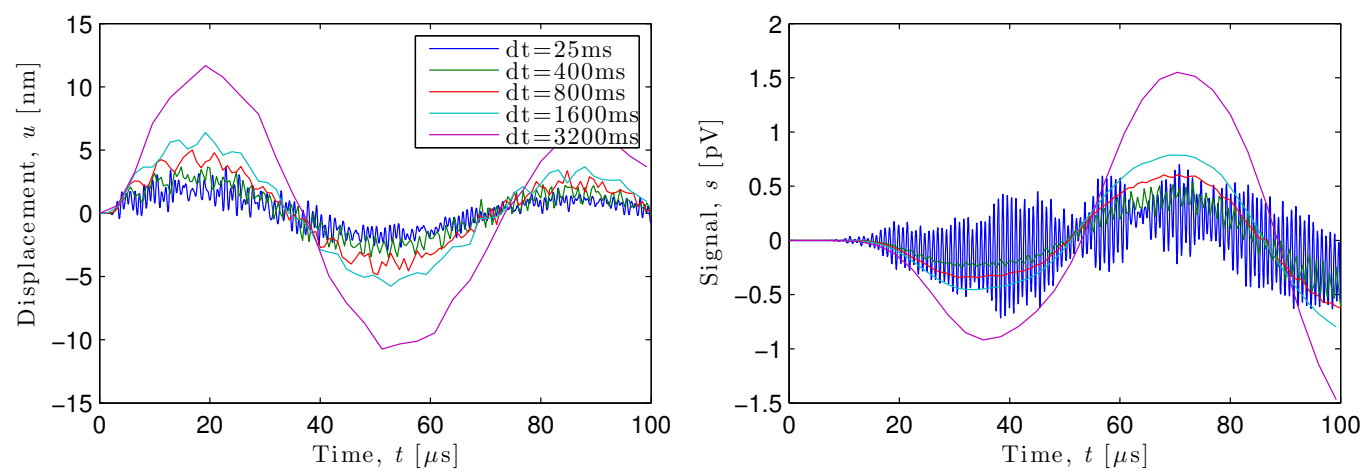

Figure 2.7: High incremental time time step convergence

found adequate for convergence is made one second refinement four parameters $m_{1}, m_{2}, m_{3}$ and $m_{4}$ to refine the mesh and find optimal convergence.

The following chart describes the geometry of the mesh:

Starting the mesh had the following dimensions:

where $n, m$ and $q_{1}$ remained intact in the refinement to maintain the boundary conditions of the model. Ten configurations were evaluated in order to obtain the best refine mesh: $m_{1}, m_{2}, m_{3}$ and $m_{4}$ like (2222), (1422), (1242), (1224), (1111), (2211), (1211), (1121), (1112) and (2422), 


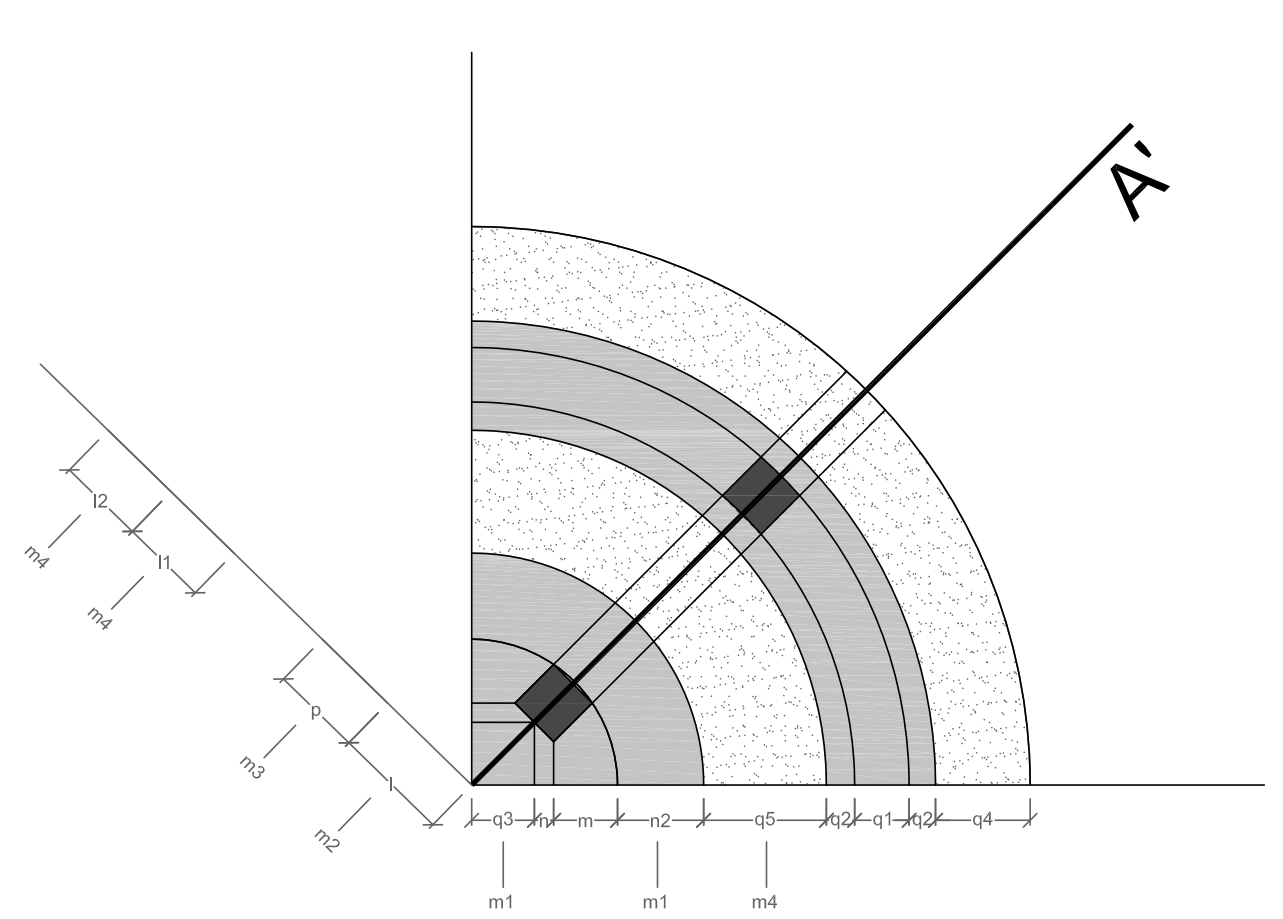

Figure 2.8: The geometry of the mesh

\subsection{Sensitivity Analysis}

\subsubsection{Sensitivity Sensor Dimensions Analysis}

Sensitivity analysis was based on varying the dimensions of the model in the following ranges showing in next table.

The first part of the study of sensitivity for the model parameters concerning the geometrical dimensions of the sensor within the ranges obtained. It follows that as the width of the piezo $A$ from 0.75 to $2.00[\mathrm{~mm}]$ increase therefore the $\mathrm{P}$-wave amplitude for high frequency as the frequency. However, the S-wave amplitude at low frequency both outside and inside the ring 


\begin{tabular}{c|cc}
\hline \hline Mesh Parameters & First dimensions & Refine mesh \\
\hline$q_{3}$ & 2 & $2 m_{1}$ \\
$n$ & 1 & 1 \\
$m$ & 1 & 1 \\
$n_{2}$ & 1 & $1 m_{1}$ \\
$q_{5}$ & 8 & $4 m_{4}$ \\
$q_{2}$ & 1 & 1 \\
$q_{1}$ & 1 & 1 \\
$q_{4}$ & 3 & $1 m_{4}$ \\
$l$ & 6 & $3 m_{2}$ \\
$p$ & 4 & $2 m_{3}$ \\
$l_{1}$ & 6 & $3 m_{4}$ \\
$l_{2}$ & 6 & $3 m_{4}$ \\
\hline \hline
\end{tabular}

Table 2.5: Previous mesh dimensions

is indifferent to this range. The same applies to the thickness of piezoelectric $B$, as increases of 0.75 to $2.00[\mathrm{~mm}]$ increase both the $\mathrm{P}$-wave amplitude for high frequency and frequency. However, the S-wave amplitude at low frequency both outside and inside the ring is indifferent to this range.

In the case of the range of values obtained for the piezoelectric length $L$ of 0.50 to $4.00[\mathrm{~mm}]$ as we increase this range the amplitude of $\mathrm{P}$-waves in ring decreases as the frequency, amplitude $\mathrm{S}$ waves at low frequency and amplitude of $\mathrm{S}$ waves in the ring at low frequency.

Thereafter, we analyze the sensitivities regarding design ranges for the sensor geometry. Denoting by $d d$ the distance from the center of the sensor transmitter to the center of the piezoelectric, to vary the range from 1.50 to $3.50[\mathrm{~mm}]$, we find that with increasing this distance, reduce the amplitude of the $\mathrm{P}$ waves to high frequency and the frequency increases, but is 


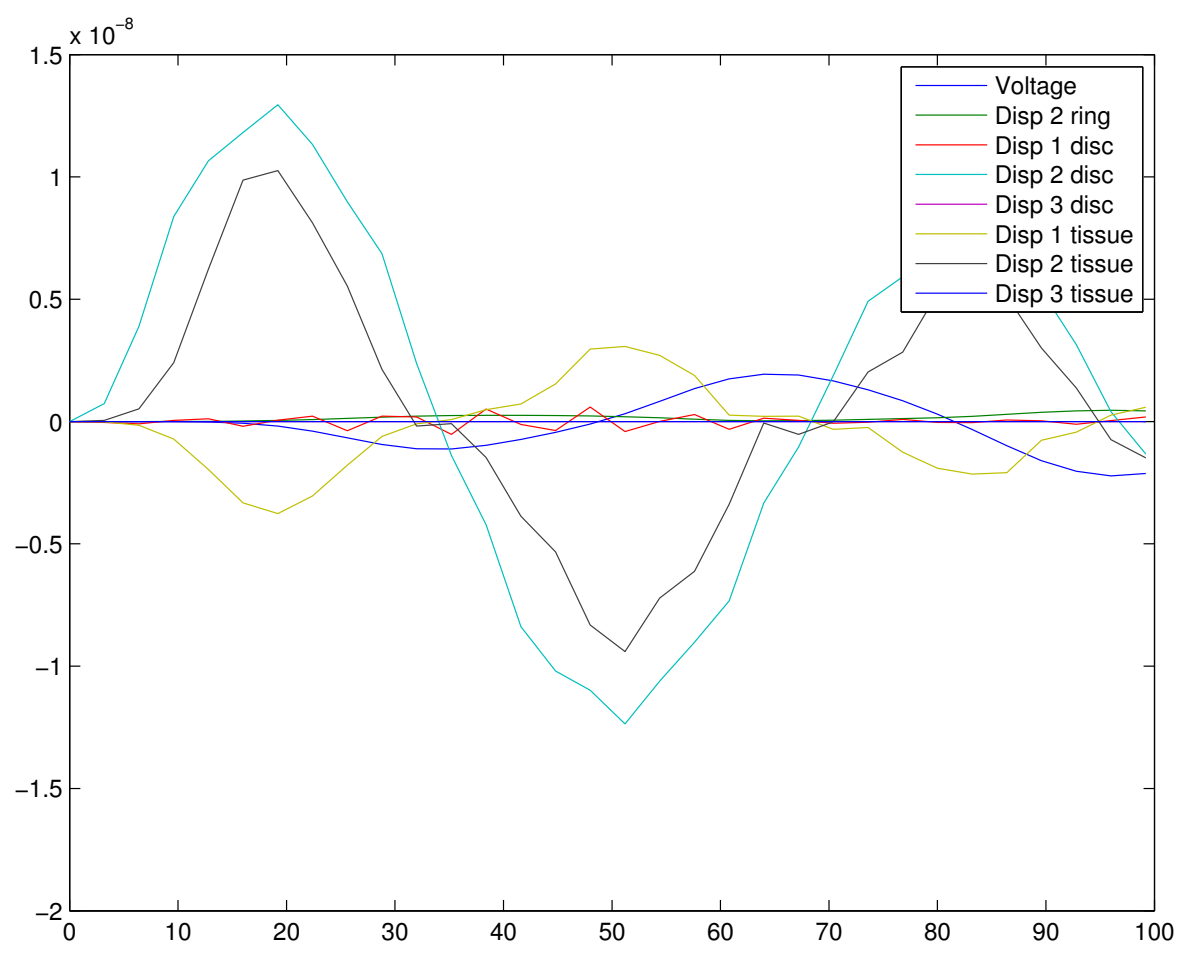

Figure 2.9: Mesh convergence

irrelevant in this range for the $\mathrm{S}$ wave amplitude at low frequency.

The following sensitivity analysis model parameter $d s$ regarding the distance from the center of the sensor transmitter to the sensor outside of reception, differs regarding others. The range of distances ranging from 1.75 to $5.75[\mathrm{~mm}]$, and with increasing amplitude of high frequency waves $\mathrm{P}$ decreases, however this distance increases as the frequency is directly proportional, as happens with the amplitude of waves at low frequency $\mathrm{S}$. The voltage signal increases as the outer radius distance.

The next parameter to be analyzed is $d R$ regarding the inner radius from the center of the transmission sensor to the center of the piezoelectric sensor on the ring. Here, the range varies between 11.5 and $17[\mathrm{~mm}]$. There is no significant change by varying the dimensions of the geometry of this distance as increases established ranges except that frequency decreases under this assumption.

The following parameter was studied for which sensitivity analysis was $e$ 


\begin{tabular}{c|ccc}
\hline \hline Design Parameters & Range & Reference $[\mathrm{mm}]$ & Label \\
\hline Width piezo & {$[0.75,2]$} & 1 & $\mathrm{~A}$ \\
Length piezo & {$[0.5,2]$} & 1 & $\mathrm{~B}$ \\
Thickness piezo & {$[0.5,4]$} & 2 & $\mathrm{~L}$ \\
Inner distance & {$[1.5,3.5]$} & 2.5 & $\mathrm{dd}$ \\
Outer radius & {$[1.75,5.75]$} & 4.25 & $\mathrm{ds}$ \\
Inner radius & {$[11.5,17]$} & 13 & $\mathrm{dR}$ \\
Length outer sensor & {$[1.5,2.5]$} & 2 & $\mathrm{e}$ \\
Steel thickness & {$[3,13]$} & 8 & $\mathrm{Lz}$ \\
\hline \hline
\end{tabular}

Table 2.6: Ranges of design dimensions
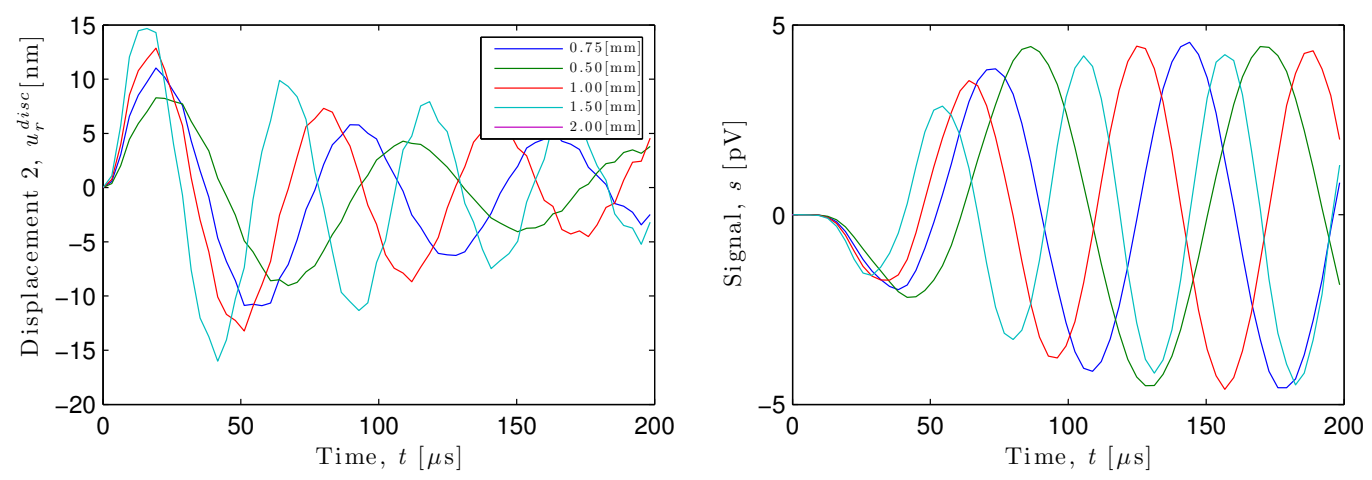

Figure 2.10: Sensitivity width piezo total time 200 radial displacement disc and voltage signal

defined as the width of the outer ring is receiving sensor. The ranges studied varied between 1.50 and $2.50[\mathrm{~mm}]$ and found no significant differences in the outcome of the movement both in the internal sensor as in the ring or in the amplitudes of the signal voltages in the total time estimated 200[ mus]. Notably only the $\mathrm{S}$ wave amplitude at low frequency in radial displacement decreases as this distance.

Finally parameter $L z$ sensitivity was analyzed which is defined as the thickness of steel ring receiver sensor. The ranges taken into account in the study were from 1.50 to $2.50[\mathrm{~mm}]$, and it follows that as the thickness 

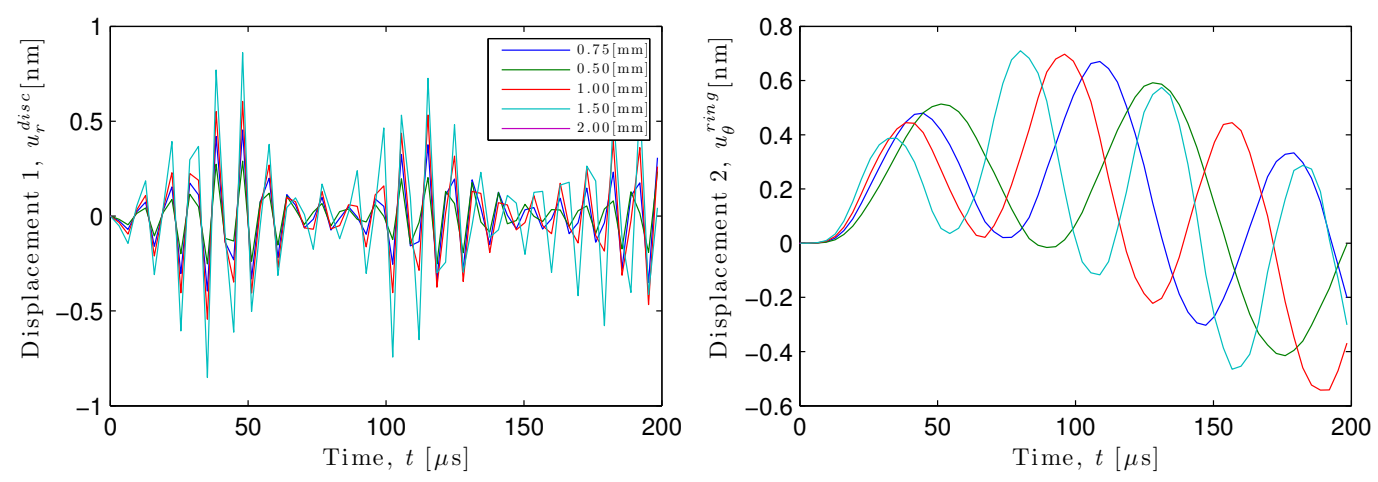

Figure 2.11: Sensitivity width piezo total time 200 turn displacement disc and radial displacement ring
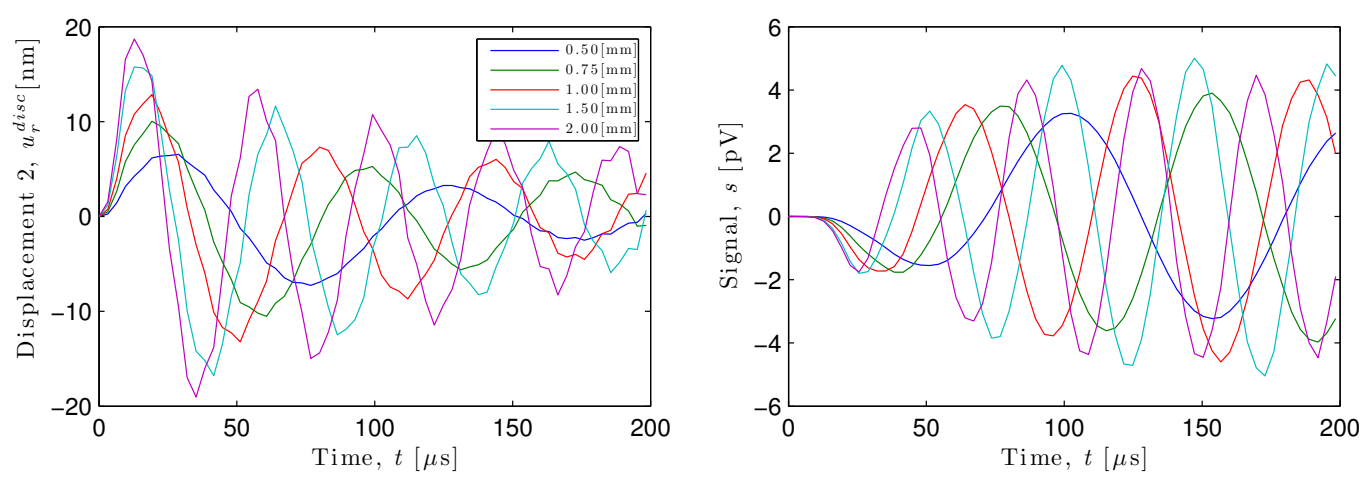

Figure 2.12: Sensitivity thickness piezo total time 200 radial displacement disc and voltage signal

increases of reception sensor steel decreases the $\mathrm{P}$ wave amplitude high frequency, just as the frequency decreases and so does the amplitude of waves at low frequency S both radially and circumferentially. The amplitude signal voltage hardly changes for noticing this range one is a small delay in time to the highest values.

Outside the field of obstetrics and gynecology, which is the first applied target on which this project focuses, a technology for diagnosis based on mechanical properties has a vast potential for other tissues, like breast or prostate tumors or liver disorders. As an example, breast cancer screening faces an important limitation due to the ionizing nature of the established mammography. This has motivated several scientists to propose various ultrasound-based diagnosis techniques. An example of the success of quan- 

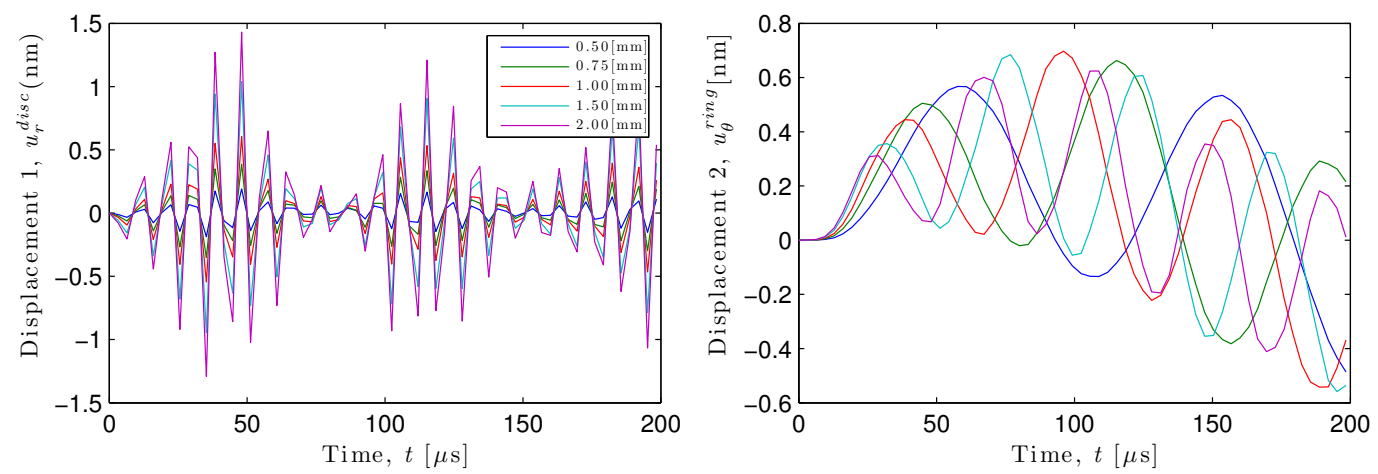

Figure 2.13: Sensitivity thickness piezo total time 200 turn displacement disc and radial displacement ring
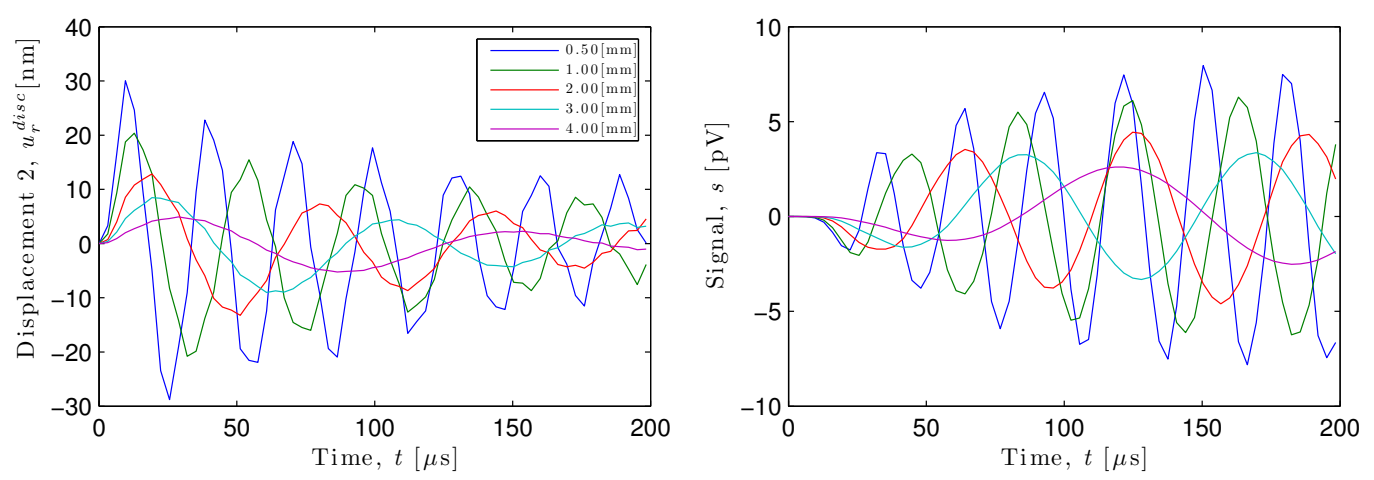

Figure 2.14: Sensitivity length piezo total time 200 radial displacement disc and voltage signal

titative sonography is given by Yonetsu[25], who show that quantitative techniques provide effective results in direct diagnosis of differentiation in benign or malignant tumors and their type. Since nodules show up very strongly by a drastic consistency change, the proposed diagnosis technology based on mechanical properties could have a strong impact on several health issues and become an excellent candidate for future research.

Another potential is to create and publish a database of in vivo mechanical properties of various tissues and organs, which is currently unavailable and much needed by the biomechanics scientific community. These examples envisage the scientific and technological long term horizon of the idea behind this project. 

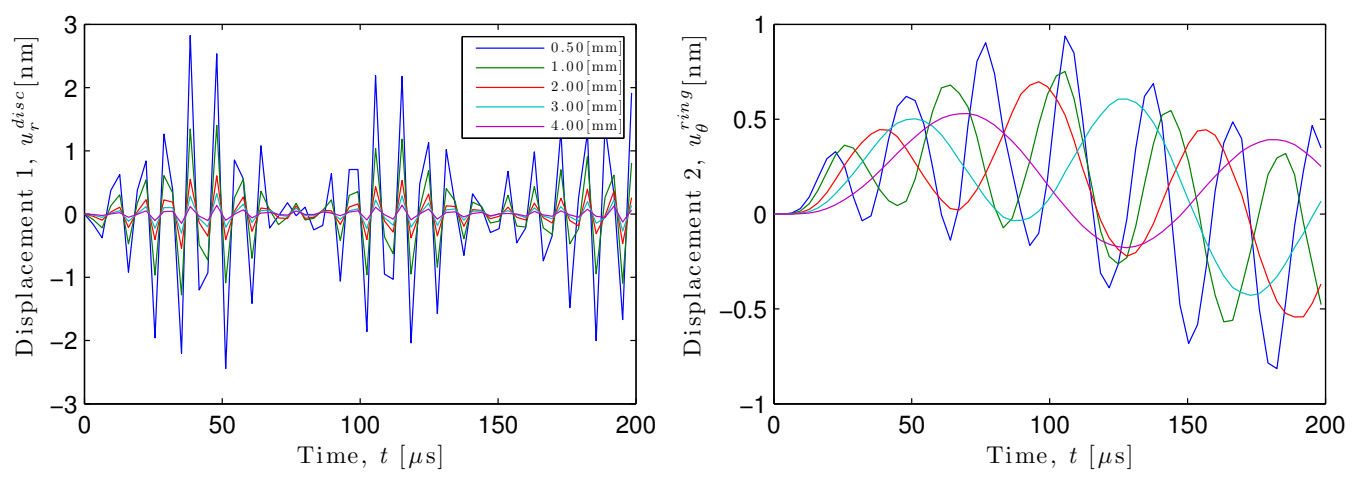

Figure 2.15: Sensitivity length piezo total time 200 turn displacement disc and radial displacement ring

\begin{tabular}{c|cccc}
\hline \hline Cause $/$ Effect & $A^{C} P(H F)$ & $\omega($ Frequency $)$ & $A S(L F)$ & $A^{C} S(L F)$ \\
\hline$A \uparrow$ & $\uparrow$ & $\uparrow$ & - & - \\
$B \uparrow$ & $\uparrow$ & $\uparrow$ & - & - \\
$L \uparrow$ & $\downarrow$ & $\downarrow$ & $\downarrow$ & $\downarrow$ or $(-)$ \\
\hline \hline
\end{tabular}

Table 2.7: Cause-effect table of ranges and amplitudes

\subsubsection{Sensitivity Tissue Analysis}

Hereinafter, the calculation of the sensitivity of the model parameters relating to the tissue is developed, both of dermal tissue layer and the layer of connective tissue, which were chosen as significant within the B model. The parameters where sensitivity was measured were the S-wave velocity in both dermic tissue and connective tissue P-wave speed both the dermic tissue and the connective tissue, besides the thickness of the connective tissue and dermic tissue, checking as varying the voltage signal, and both longitudinal and radial displacements. The ranges were chosen by central reference to those included in the simplified analytical model and were as follows:

For the $\mathrm{S}$ wave velocity of $[20,380][\mathrm{m} / \mathrm{s}]$, for the speed of P-waves propagating in tissue $[1200,1800][\mathrm{m} / \mathrm{s}]$ and for the thicknesses layers of dermic tissue and connective tissue $[0.3,0.7][\mathrm{mm}]$. 

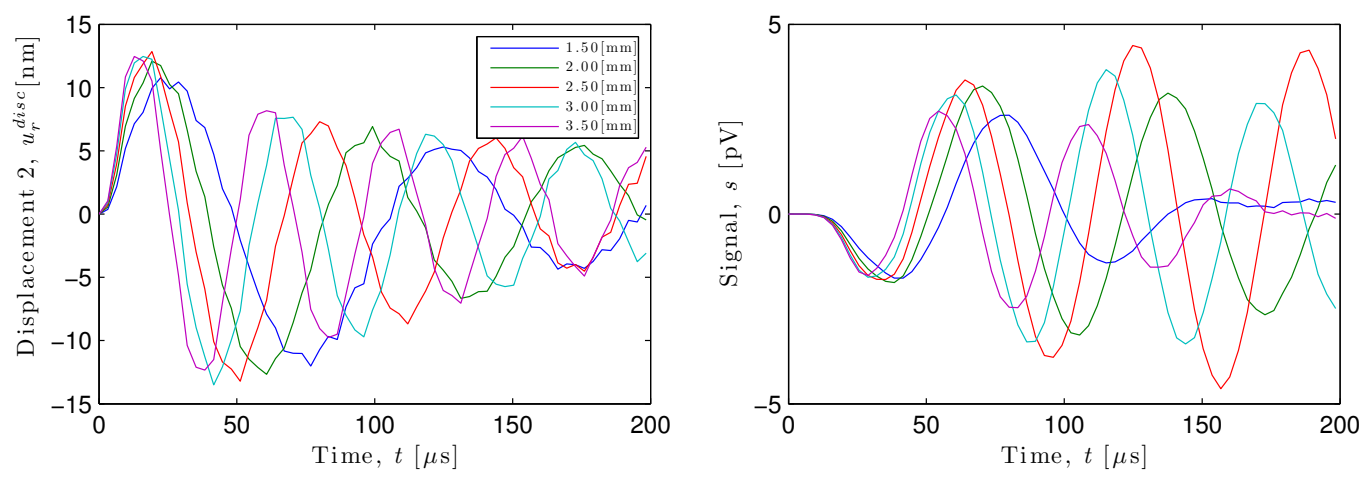

Figure 2.16: Sensitivity inner distance total time 200 radial displacement disc and voltage signal
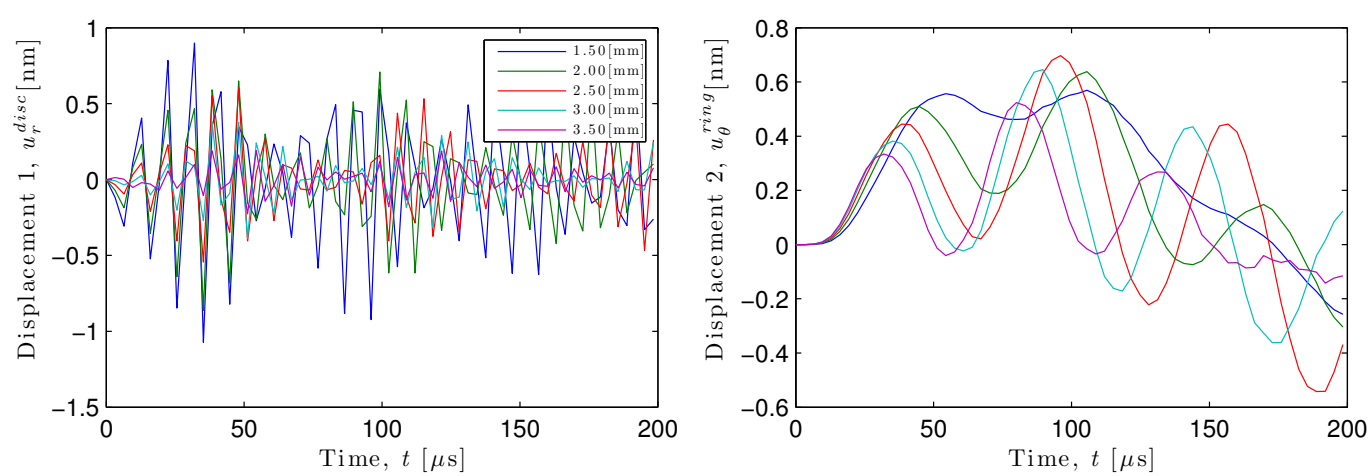

Figure 2.17: Sensitivity inner distance total time 200 turn displacement disc and radial displacement ring

By varying the speed of $\mathrm{S}$ waves from modifying the Young's modulus and Poisson's ratio of dermal tissue, we find that the high-frequency amplitude of $\mathrm{P}$ waves is independent of the range of values used in the sensitivity analysis, however the frequency increases with increase the speed of $\mathrm{S}$ waves, so does the amplitude of $\mathrm{S}$ waves at low frequency in the radial displacement of the ring, but the $\mathrm{S}$ wave amplitude low frequency decreases as the speed increases in $\mathrm{S}$ waves in the radial displacement of the disc.

By varying the speed of $\mathrm{S}$ waves from modifying the Young's modulus and Poisson's ratio of dermal tissue, we find that the high-frequency amplitude of $\mathrm{P}$ waves is independent of the range of values used in the sen- 

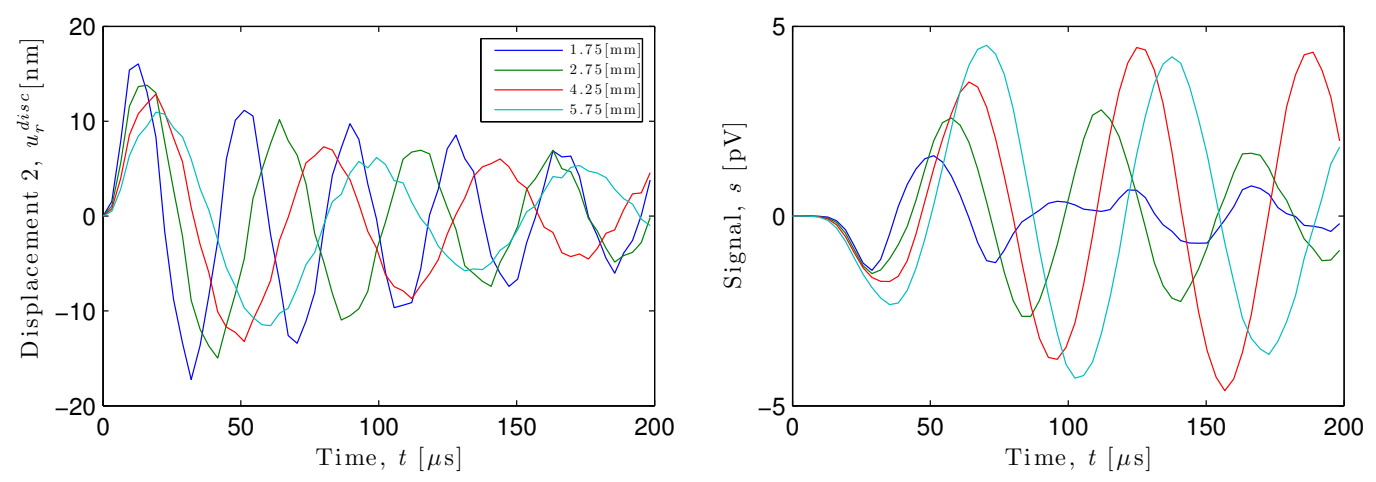

Figure 2.18: Sensitivity outer radius total time 200 radial displacement disc and voltage signal
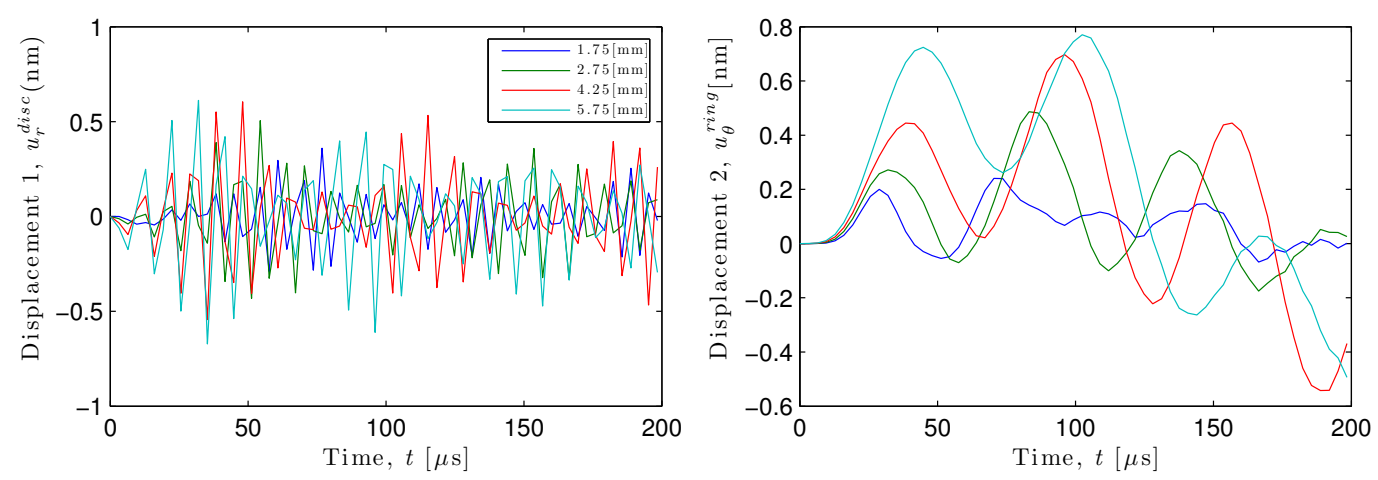

Figure 2.19: Sensitivity length outer radius time 200 turn displacement disc and radial displacement ring

sitivity analysis, however the frequency increases with increase the speed of $\mathrm{S}$ waves, so does the amplitude of $\mathrm{S}$ waves at low frequency in the radial displacement of the ring, but the $\mathrm{S}$ wave amplitude low frequency does not vary with the speed increases in $\mathrm{S}$ waves in the radial displacement of the disc.

Hereinafter, the calculation of the sensitivity of the model parameters relating to the tissue is developed, both of dermal tissue layer and the layer of connective tissue, which were chosen as significant within the B model. The parameters where sensitivity was measured were the S-wave velocity in both dermic tissue and connective tissue $\mathrm{P}$-wave speed both the dermic tissue and the connective tissue, besides the thickness of the connective 

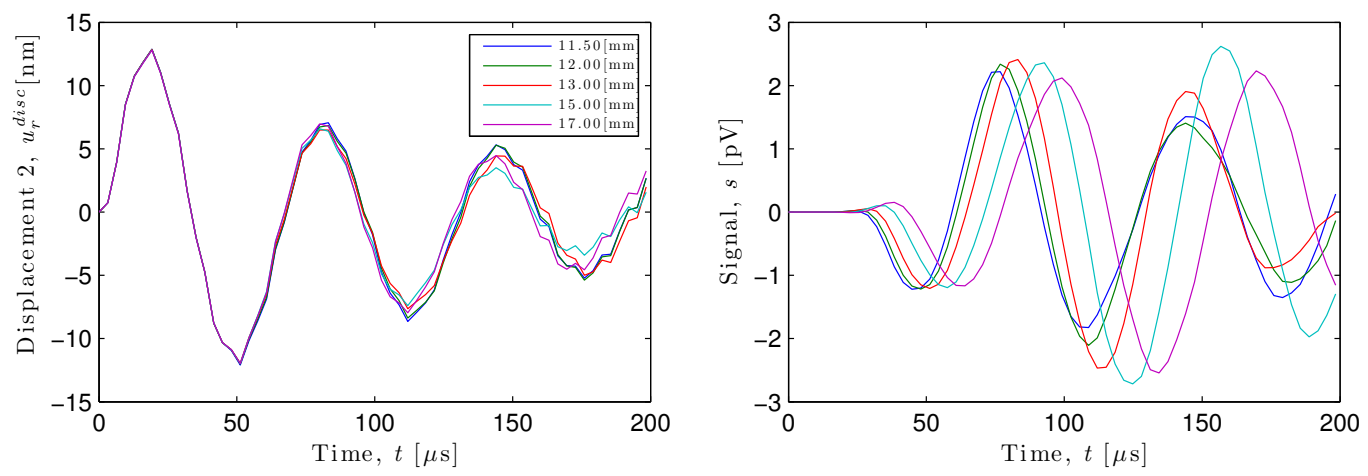

Figure 2.20: Sensitivity inner radius total time 200 radial displacement disc and voltage signal
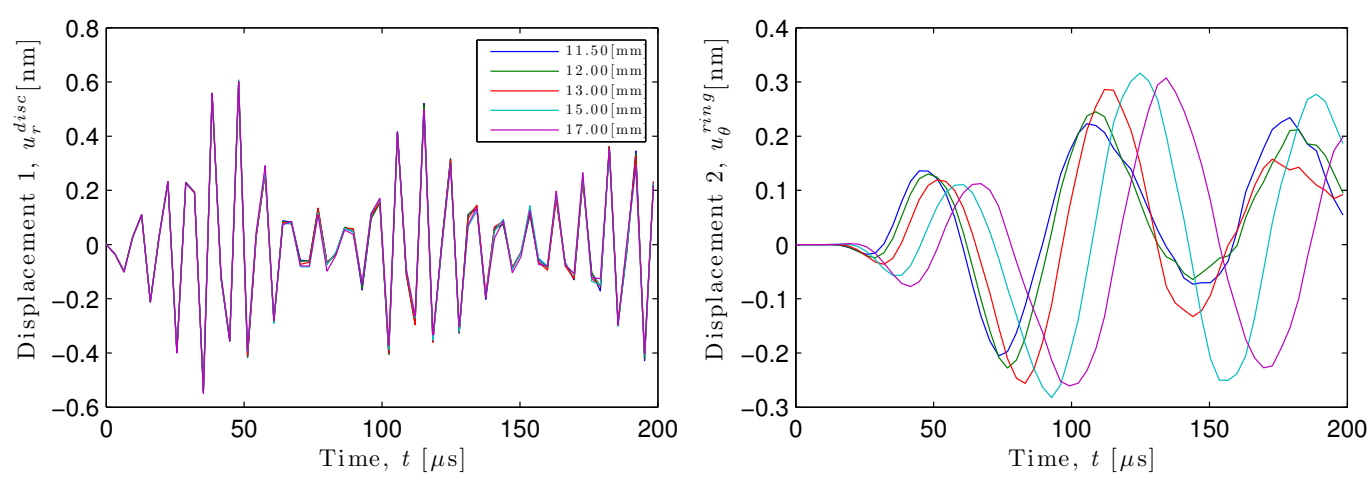

Figure 2.21: Sensitivity length inner radius time 200 turn displacement disc and radial displacement ring

tissue and dermic tissue, checking as varying the voltage signal, and both longitudinal and radial displacements. The ranges were chosen by central reference to those included in the simplified analytical model and were as follows:

Hereinafter, the calculation of the sensitivity of the model parameters relating to the tissue is developed, both of dermal tissue layer and the layer of connective tissue, which were chosen as significant within the B model. The parameters where sensitivity was measured were the S-wave velocity in both dermic tissue and connective tissue $\mathrm{P}$-wave speed both the dermic tissue and the connective tissue, besides the thickness of the connective tissue and dermic tissue, checking as varying the voltage signal, and both longitudinal and radial displacements. The ranges were chosen by central 

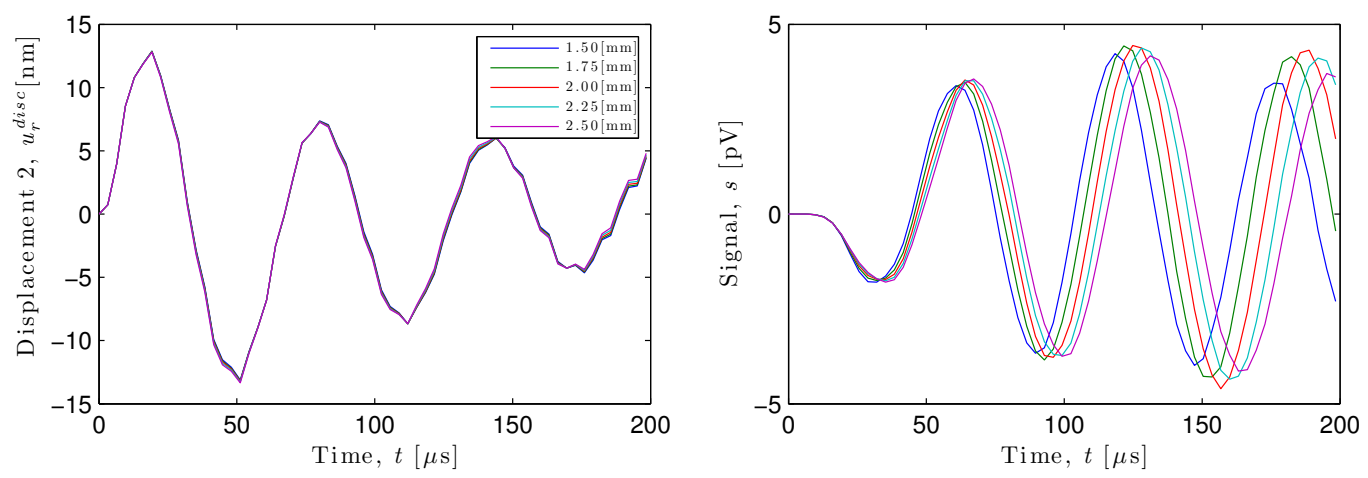

Figure 2.22: Sensitivity thickness outer sensor total time 200 radial displacement disc and voltage signal
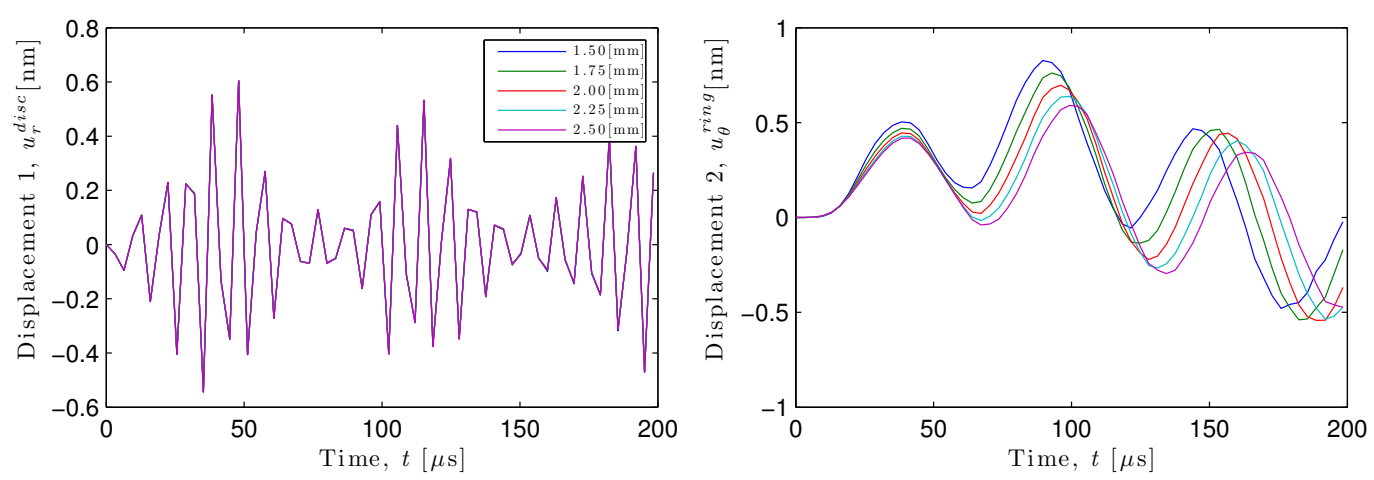

Figure 2.23: Sensitivity thickness outer sensor total time 200 turn displacement disc and radial displacement ring

reference to those included in the simplified analytical model and were as follows:

Hereinafter, the calculation of the sensitivity of the model parameters relating to the tissue is developed, both of dermal tissue layer and the layer of connective tissue, which were chosen as significant within the B model. The parameters where sensitivity was measured were the S-wave velocity in both dermic tissue and connective tissue $\mathrm{P}$-wave speed both the dermic tissue and the connective tissue, besides the thickness of the connective tissue and dermic tissue, checking as varying the voltage signal, and both longitudinal and radial displacements. The ranges were chosen by central reference to those included in the simplified analytical model and were as follows: 

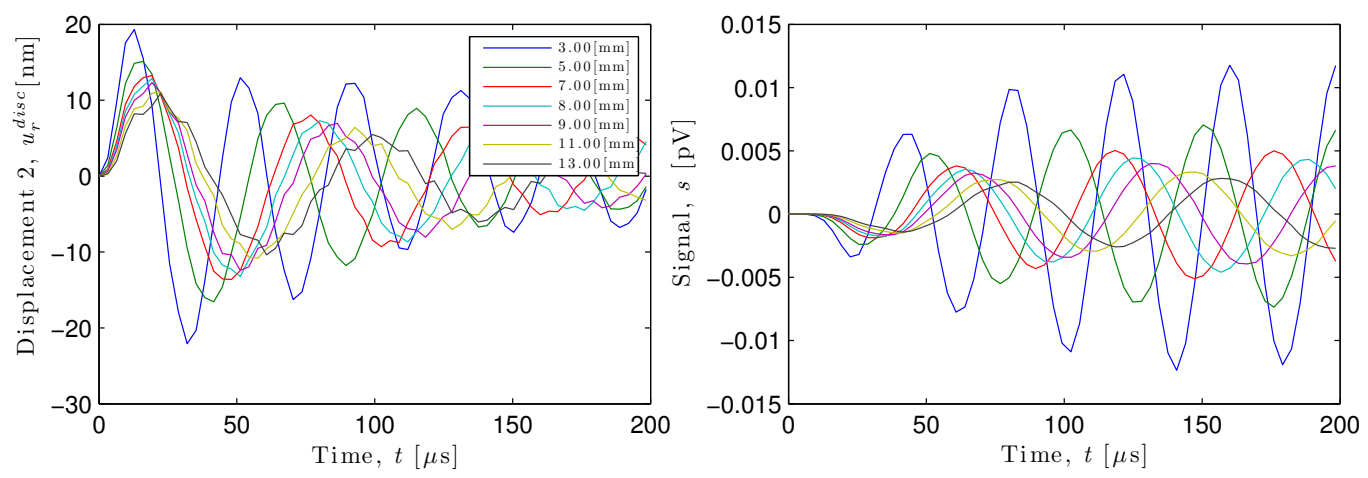

Figure 2.24: Sensitivity steel thickness total time 200 radial displacement disc and voltage signal
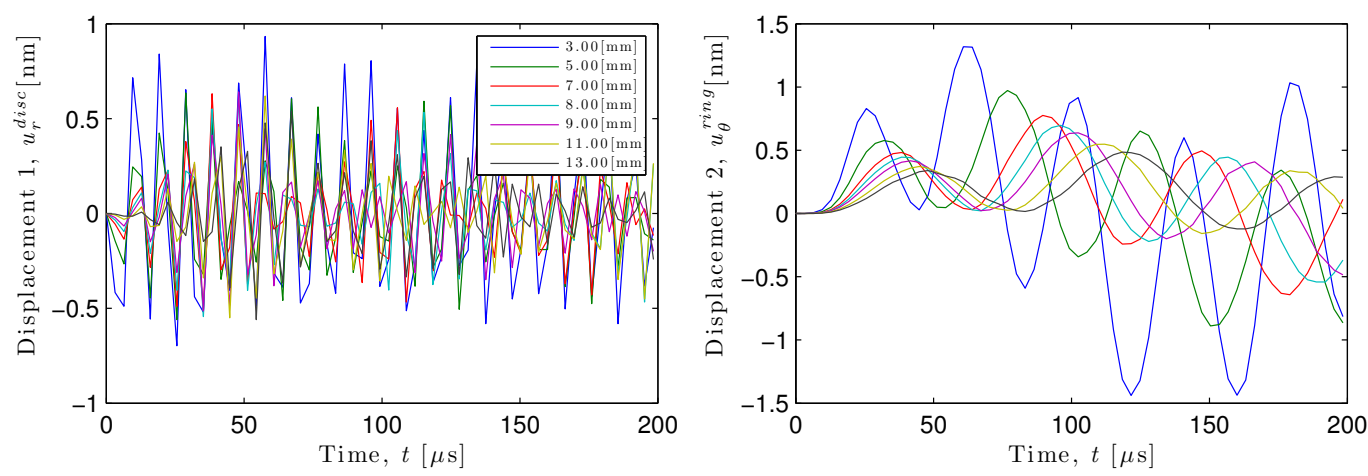

Figure 2.25: Sensitivity steel thickness total time 200 turn displacement disc and radial displacement ring

\subsection{Conclusions}




\begin{tabular}{c|cccc}
\hline \hline Cause $/$ Effect & $A^{C} P(H F)$ & $\omega($ Frequency $)$ & $A S(L F)$ & $A^{C} S(L F)$ \\
\hline$d d \uparrow$ & $\downarrow$ & $\uparrow$ & - & - \\
$d s \uparrow$ & $\downarrow$ & $\uparrow$ & $\uparrow$ & $\uparrow$ \\
$d R \uparrow$ & - & $\downarrow$ & - & - \\
$e \uparrow$ & - & - & $\downarrow$ & - \\
$L z \uparrow$ & $\downarrow$ & $\downarrow$ & $\downarrow$ & $\downarrow$ \\
\hline \hline
\end{tabular}

Table 2.8: Cause-effect table of ranges and amplitudes for piezoelectric geometry
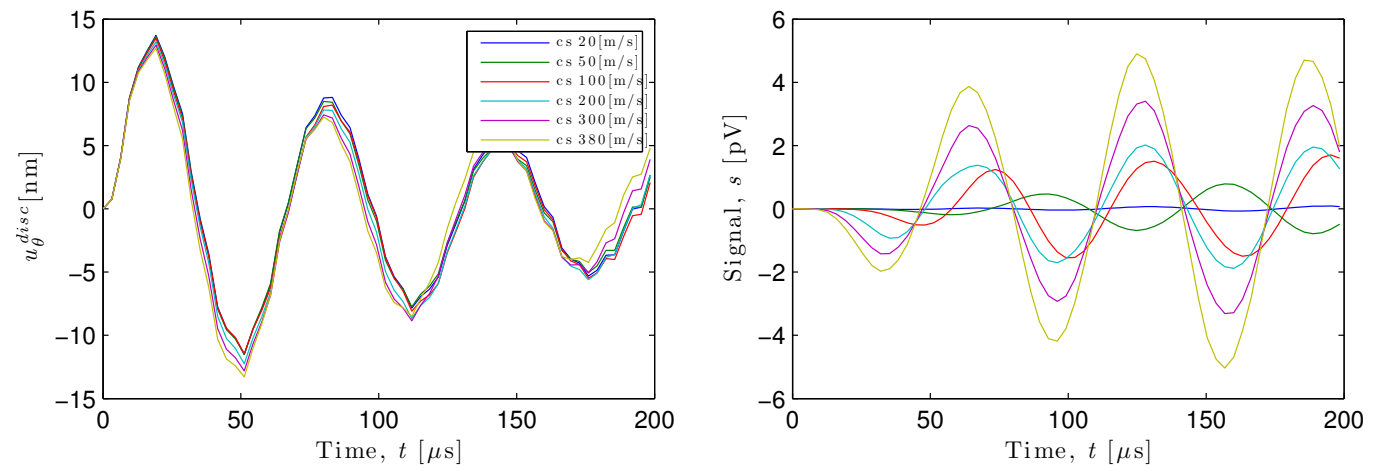

Figure 2.26: Sensitivity of dermic tissue total time 200 turn radial displacement disc and voltage signal varying second wave speed
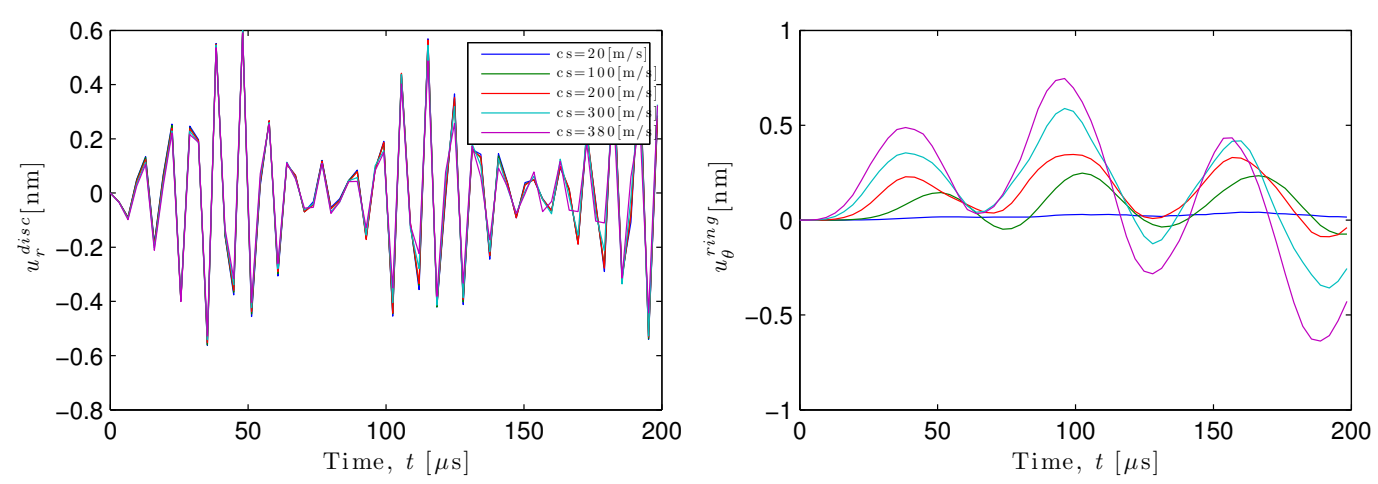

Figure 2.27: Sensitivity of dermic tissue total time 200 turn displacement disc and radial displacement ring varying second wave speed 

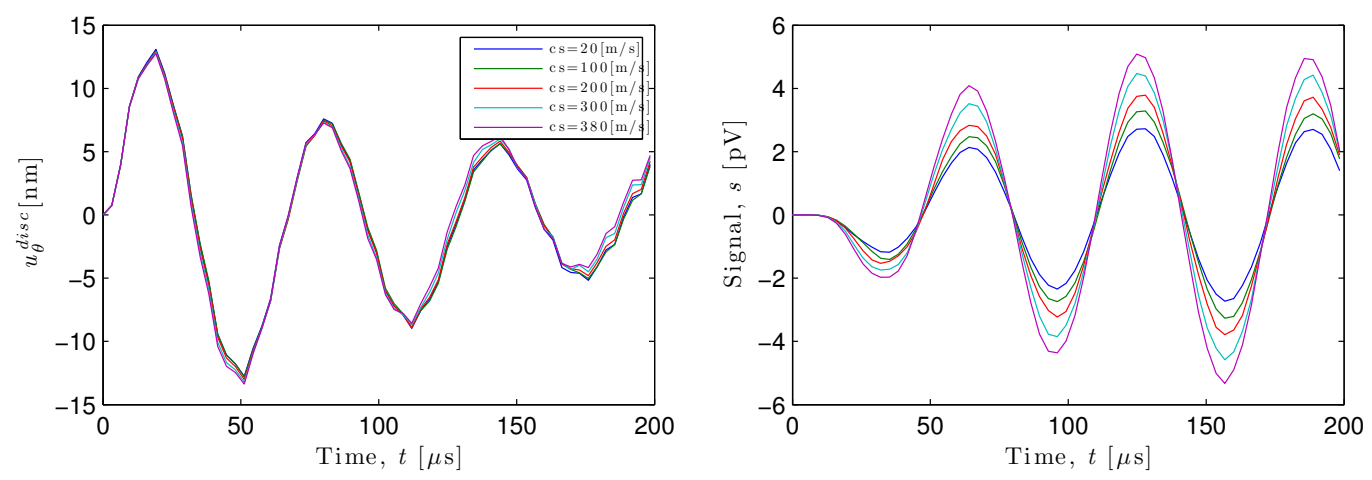

Figure 2.28: Sensitivity of connective tissue total time 200 turn radial displacement disc and voltage signal varying second wave speed
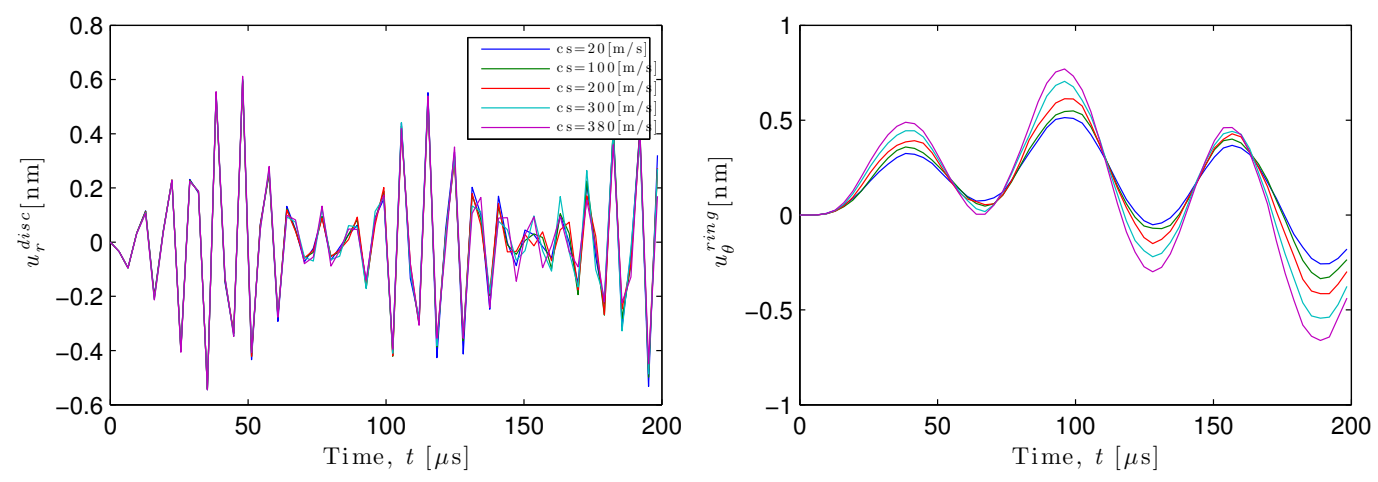

Figure 2.29: Sensitivity of connective tissue total time 200 turn displacement disc and radial displacement ring varying second wave speed
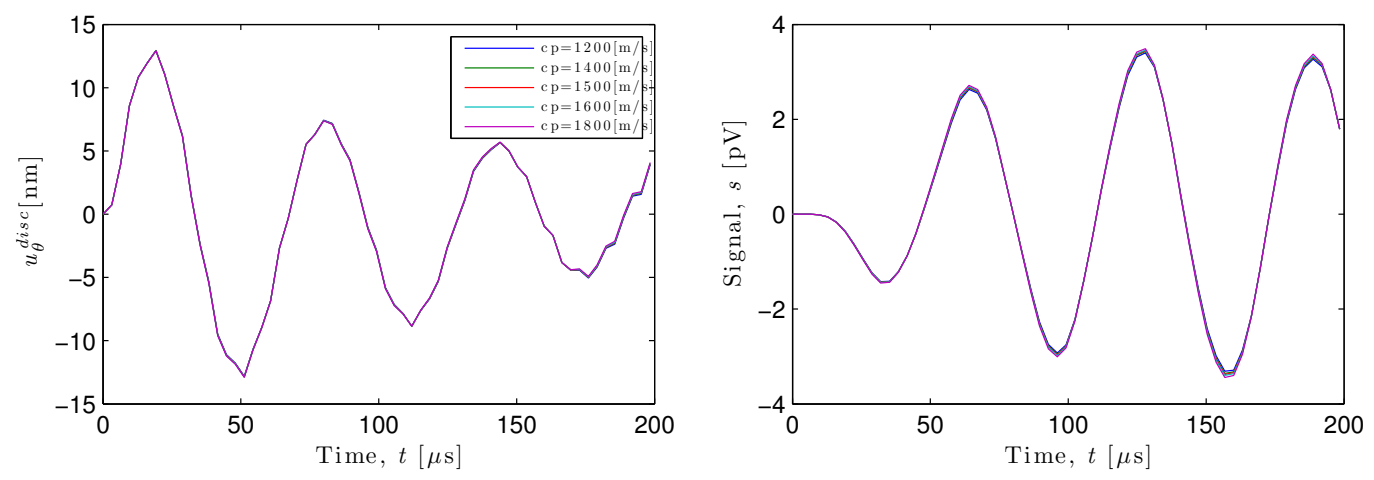

Figure 2.30: Sensitivity of dermic tissue total time 200 turn radial displacement disc and voltage signal varying primary wave speed 

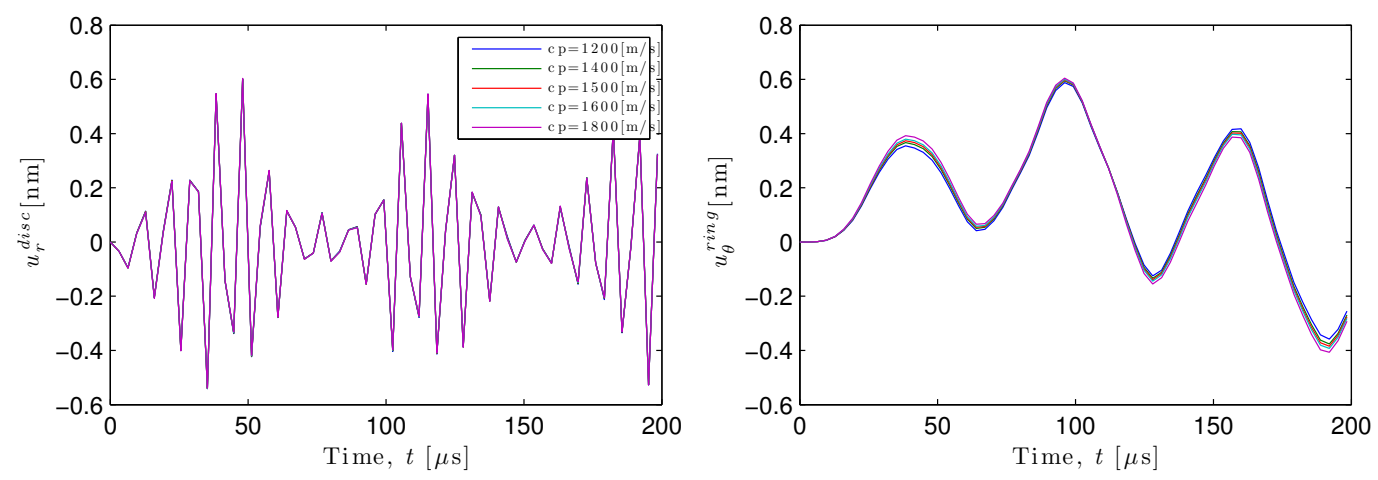

Figure 2.31: Sensitivity of dermic tissue total time 200 turn displacement disc and radial displacement ring varying primary wave speed
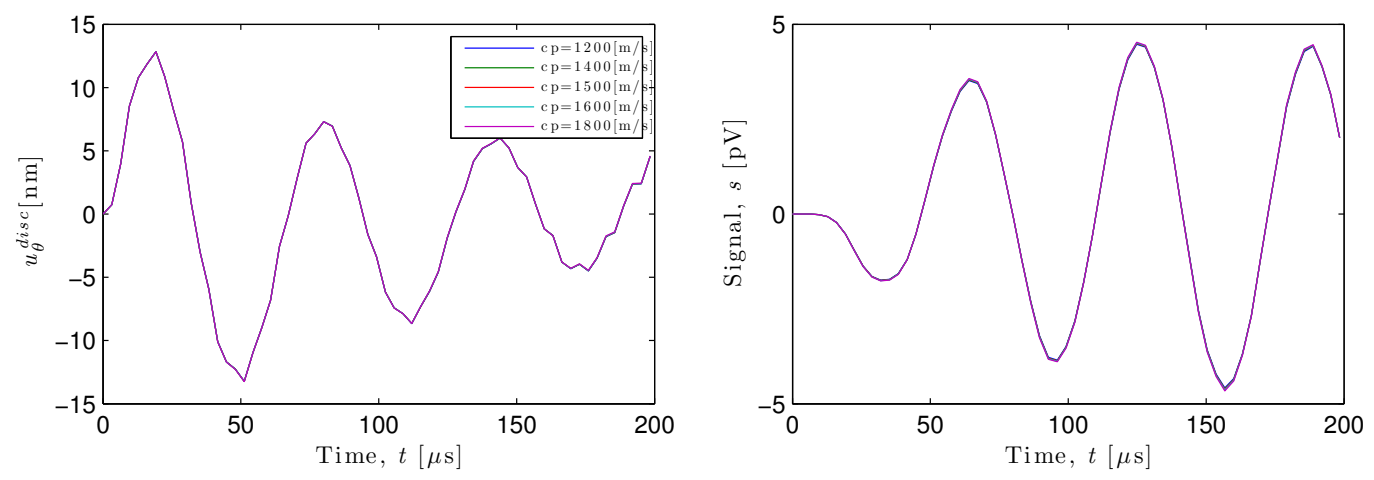

Figure 2.32: Sensitivity of connective tissue total time 200 turn radial displacement disc and voltage signal varying primary wave speed
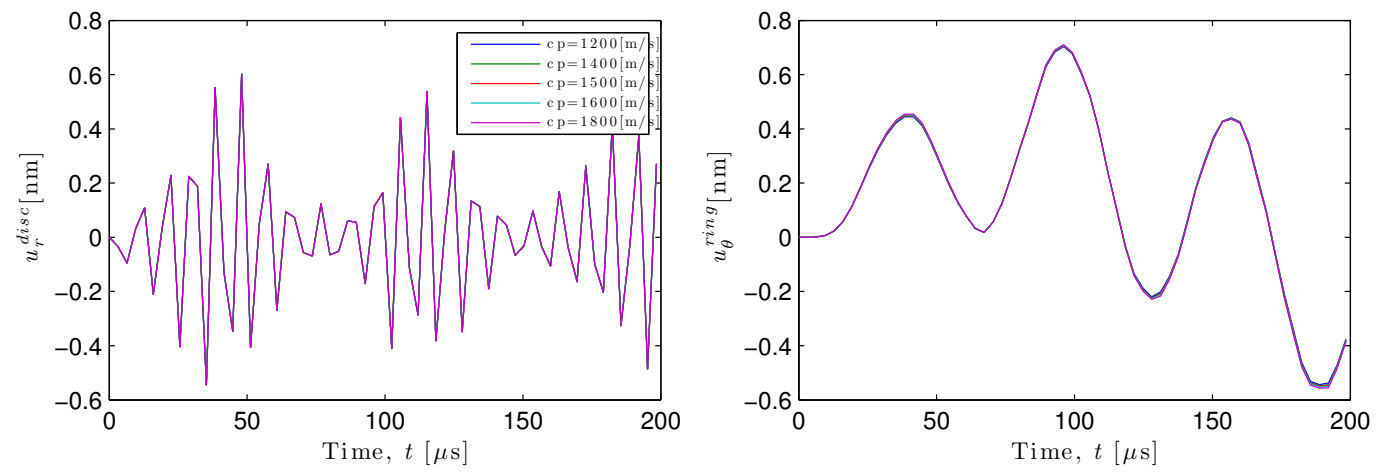

Figure 2.33: Sensitivity of connective tissue total time 200 turn displacement disc and radial displacement ring varying primary wave speed 

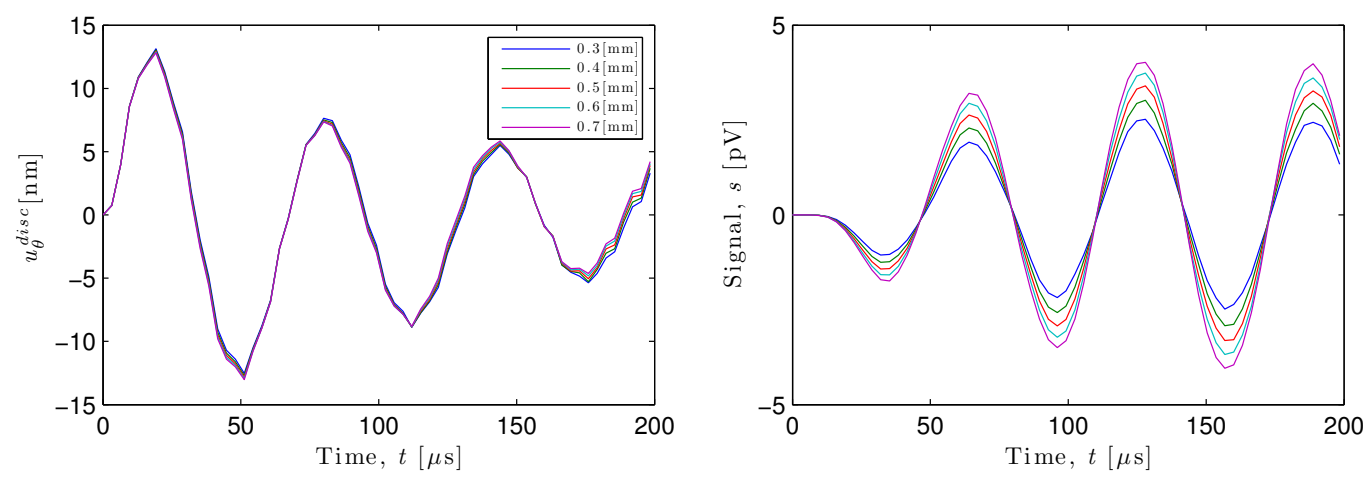

Figure 2.34: Sensitivity of dermic tissue thickness total time 200 turn radial displacement disc and voltage signal
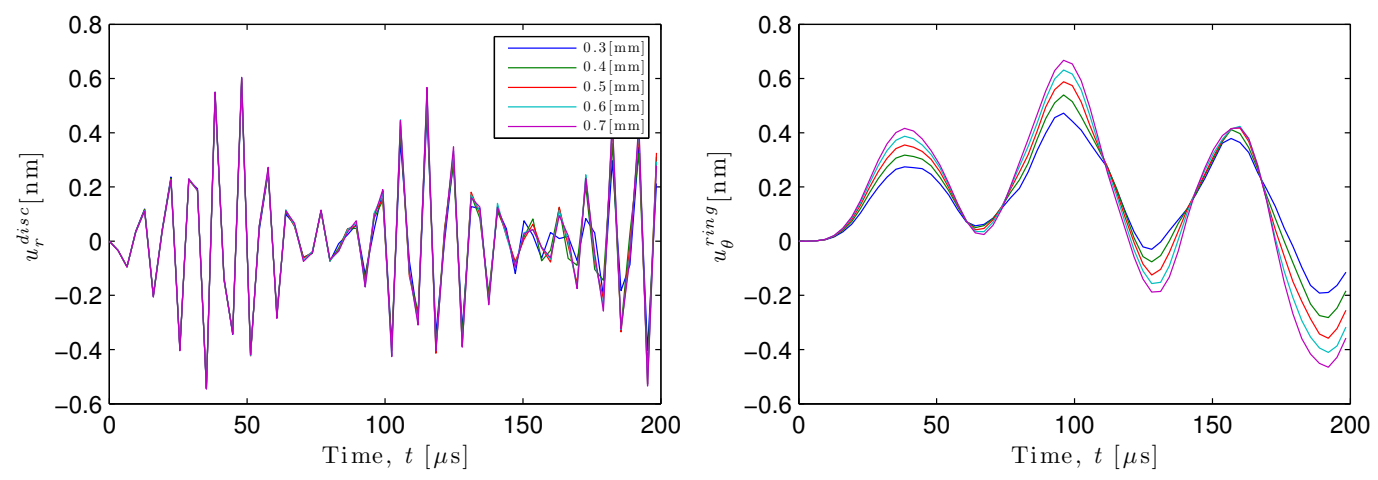

Figure 2.35: Sensitivity of dermic tissue thickness total time 200 turn displacement disc and radial displacement ring
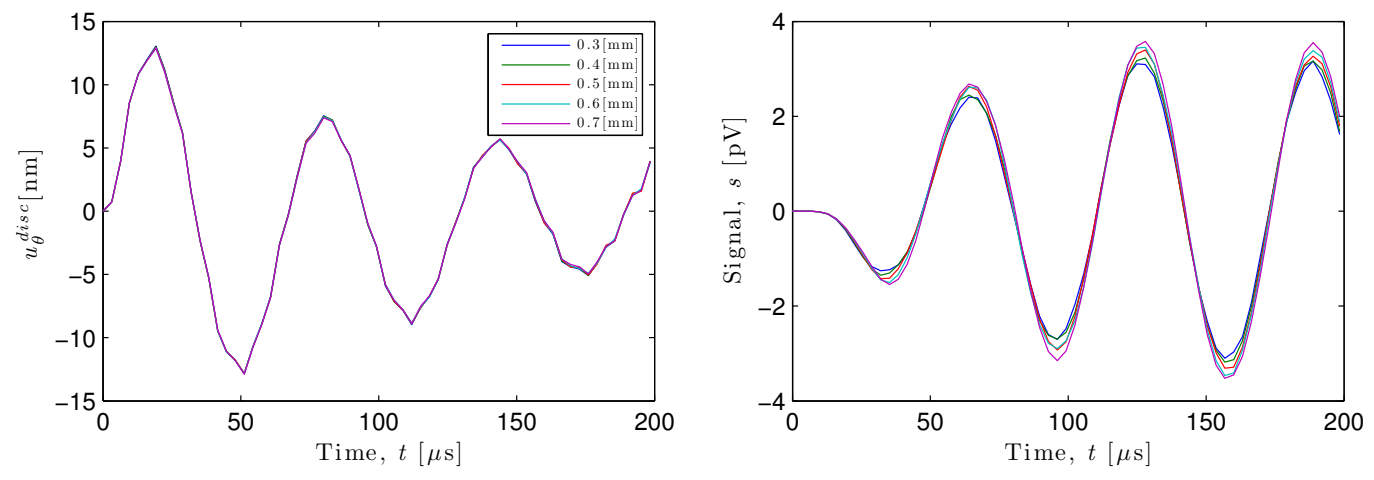

Figure 2.36: Sensitivity of connective tissue thickness total time 200 turn radial displacement disc and voltage signal 

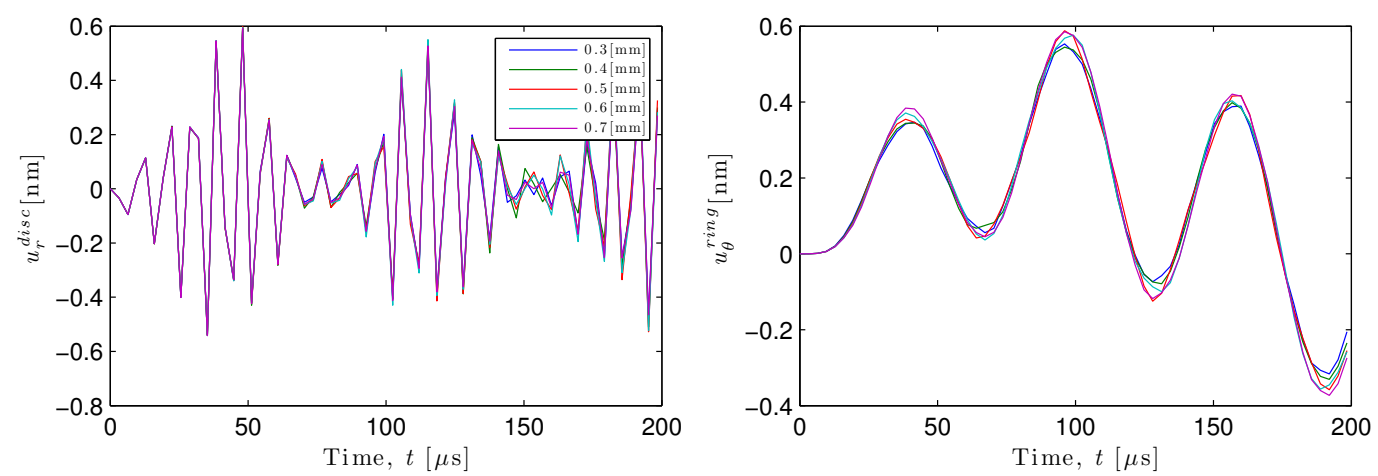

Figure 2.37: Sensitivity of connective tissue thickness total time 200 turn displacement disc and radial displacement ring

\begin{tabular}{c|cccc}
\hline \hline Cause/Effect & $A^{C} P(H F)$ & $\omega($ Frequency $)$ & $A S(L F)$ & $A^{C} S(L F)$ \\
\hline$c_{s}$ dermic $\uparrow$ & - & $\uparrow$ & $\downarrow$ & $\uparrow$ \\
$c_{s}$ connective $\uparrow$ & - & $\uparrow$ & - & $\uparrow$ \\
$c_{p}$ dermic $\uparrow$ & - & - & - & - \\
$c_{p}$ connective $\uparrow$ & - & - & - & - \\
Tz $\uparrow$ & - & - & - & $\uparrow$ \\
$\operatorname{Tc} \uparrow$ & - & - & - & $(-)$ or $\uparrow$ \\
\hline \hline
\end{tabular}

Table 2.9: Cause-effect table of ranges and amplitudes for transducers geometry

\begin{tabular}{c|cc}
\hline \hline Design Parameters & Optimal dimensions $[\mathrm{mm}]$ & Label \\
\hline Width piezo & $0.75-1$ & $\mathrm{~A}$ \\
Length piezo & $0.75-1$ & $\mathrm{~B}$ \\
Thickness piezo & 3 & $\mathrm{~L}$ \\
Inner distance & $2-2.5$ & $\mathrm{dd}$ \\
Outer radius & 4.25 & $\mathrm{ds}$ \\
Inner radius & 15 & $\mathrm{dR}$ \\
Length outer sensor & 2 & $\mathrm{e}$ \\
Steel thickness & 5 & $\mathrm{Lz}$ \\
\hline \hline
\end{tabular}

Table 2.10: Final dimensions of transducer 

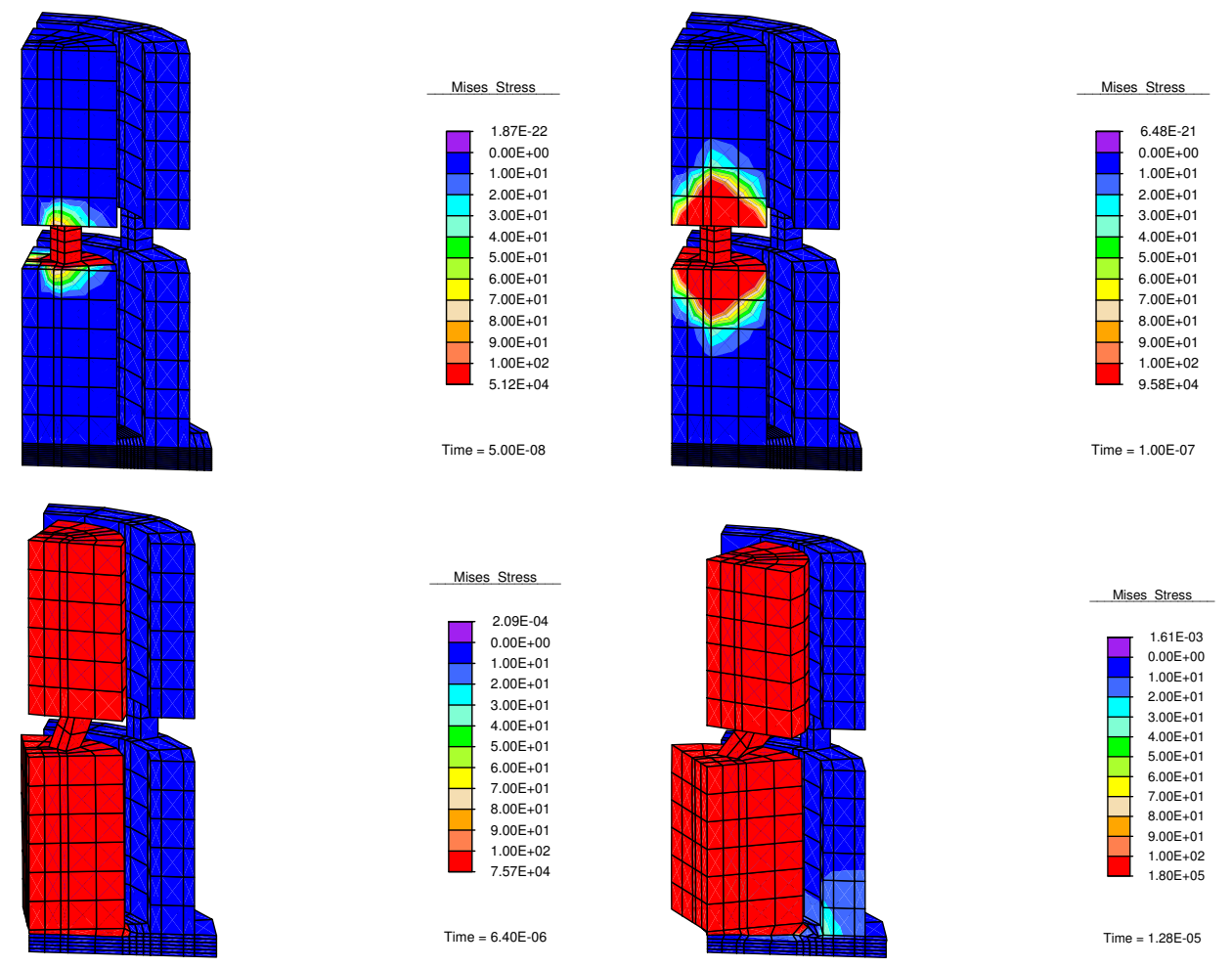

Time $=6.40 \mathrm{E}-06$

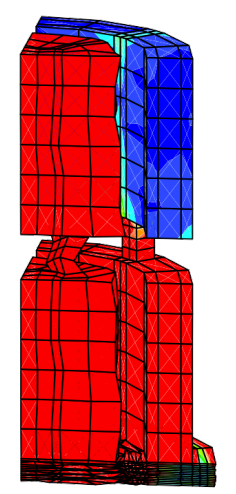

Time $=1.28 \mathrm{E}-05$
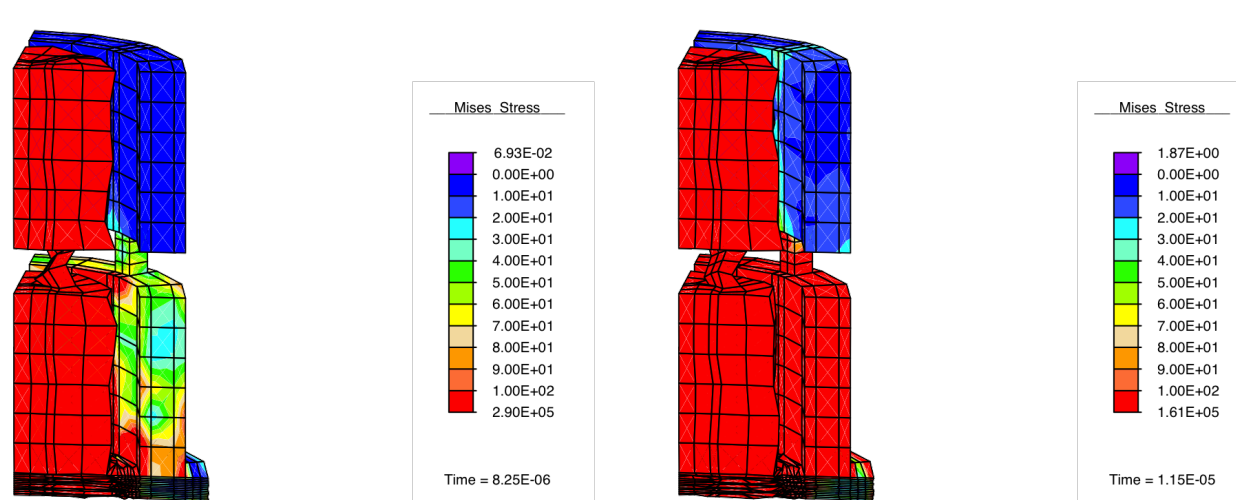

Figure 2.38: Different states of torsional transducer 


\section{Chapter 3}

\section{POD: Probability Of Detection of Pathologies in soft tissue}

Reliable quantification of the stiffness modulus of soft tissue is an open issue with relevance for the diagnostic of pathologies that appear as drastic changes in the consistency of the tissue. For this task, we propose to design a piezoelectric transducer for non-destructive testing based on an identification inverse problem, to reconstruct the stiffness modulus of the tissue using ultrasonics. Therefore, it is necessary to optimize the piezoelectric transducer model design regarding two types of parameters. On one hand the design parameters, and on the other hand the model parameters that characterize the specimen. The forward problem is obtained by performing a three-dimensional finite element simulation. The experimental measurements are simulated by adding a gaussian noise as a percentage of the RMS of the numerically predicted signals. In addition, a semi-analytical estimate of the probability of detection (POD) is developed to provide a rational criterion to optimize the experimental design. An advanced noise filtering algorithm allow us to maximize the POD. This work aims to (i) evaluate the optimal piezoelectric transducer design of the model-based POD. A second goal is (ii) a simulated experiment based on the three dimensional model 
of wave propagation generated by the proposed piezoelectric transducer design. Finally, (iii) a parametric study is carried out to extract practical parameters for final tissular applications. 


\subsection{Introduction}

The physical principle to mechanically characterize the tissue is the following. A physical magnitude is propagated along the medium to be analyzed, which distorts the wave until it is measured at an accessible surface (see Fig. 3.1). The mechanical parameters responsible for the modification of the wave can be inferred from the measured one under certain circumstances by means of the inverse problem theory discussed later. Ultrasound is chosen as the physical magnitude for several reasons. First, it is a mechanical wave, controlled by and therefore most sensitive to the mechanical parameters than any other indirect measurement. Second, the wave is generated at a low strain regime, which has been observed to be more sensitive to variations due to pathology than high strain (Matsumura et al [26]).

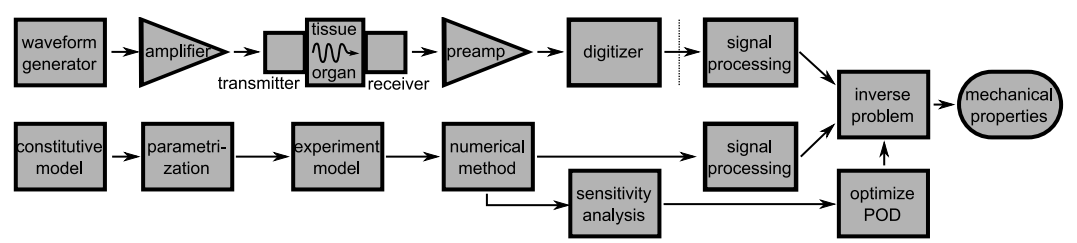

Figure 3.1: Simplified system for measuring ultrasound wave distortion through tissue and reconstruct mechanical properties.

Very scarce and indirect conclusions about mechanical properties analyzed by quantitative ultrasound have been reported specifically for cervical tissue. Bigelow et al [27] recently proposed measurement of ultrasonic attenuation through cervical tissue but concluded that too large variances in their experimental setup did not allow a statistically significant correlation with gestational age. A similar approach is recently being proposed for animals by McFarlin et al [28, 29]. These results indicate that a combination of quantitative ultrasonic parameters have the potential for extracting information for characterizing tissue condition.

Static or slow viscoelastic mechanical constitutive laws and their values were reported by Bader et al [30] for skin and by Ahuja [31] for various 
internal tissues. However, values measured at medium and high frequency seem to differ several order of magnitudes. At audible frequency dynamics, a linear viscoelastic model was proposed by Pereira et al [32] to fit the experimental observations.

\subsection{Inverse Procedure}

The problem of nondestructive characterization of mechanical tissue properties is solved by a model-based inverse problem (IP) approach that consists of two steps: (i) to excite the system applying displacement, and (ii) to measure the response (displacements). A finite element method model is used in the forward procedure that is explained in detail in the last chapter.

The inverse procedure presented aims at characterizing mechanical properties in a soft tissue (degree of degradation) and determine its extent. The testing consists of two steps: (1) to disturb a tissue with a known excitation function and (2) to measure its response at one locations in the soft tissue. We assume that the dynamic behavior of the tissue in its health and pathologies states is predictable using a well-calibrated model.

Then, the measured signal is processed to solve the inverse problem, i.e., to determine the changes in the tissue from its original state. A genetic algorithm search tool (Rus et al[33], Goldberg[34]) is used to minimize the discrepancy between the experimental readings and the numerically predicted trial response, by means of a cost functional designed to calibrate for coherent uncertainties and noise, and providing maximal robustness and sensitivity. 


\subsubsection{Cost functional}

The readings from the sensors are denoted by $\psi$ for the theoretical or synthetic case, and $\psi^{x}$ for the experimental case. A reading $\psi^{0}$ in the health state of the tissue is defined for calibration, and the measurement to analyze is defined as,

$$
\Phi=\frac{\psi-\psi^{0}}{\operatorname{RMS}\left(\psi^{0}\right)}
$$

where the RMS values are defined for a discrete function $f$ in time domain $f\left(t_{i}\right)$ at $N$ sampling points as,

$$
\operatorname{RMS}(f)=\sqrt{\frac{1}{N} \sum_{i=0}^{N-1} f\left(t_{i}\right)^{2}}
$$

A residual $\gamma$ is defined from the misfit or discrepancy $\Phi^{x}-\Phi$ between the measurements.

$$
\gamma=\left(\Phi^{x}-\Phi\right)
$$

The cost functional $f$ or fitness function is defined after a residual vector $\gamma$ of size $N_{i}$ as the quadratic form,

$$
f=\frac{1}{2}|\gamma|^{2}=\frac{1}{2} \frac{1}{N_{i}} \sum_{i=1}^{N_{i}} \gamma_{i}^{2}
$$

It is useful to define an alternative version of the cost functional denoted as $f^{l}$, with the property of improving the sensitivity while approaching the optimum, just by introducing a logarithm and a small value $\epsilon$ to ensure its existence. This definition particularly enhances the convergence speed when the minimization is tackled by with genetic algorithms or other random search algorithms (see Rus et al.[35]),

$$
f^{l}=\log (f+\epsilon)
$$




\subsubsection{Probability of detection}

The POD gives an idea of the probability that a pathology is positively detected, given a specimen, a pathology size and some noise and system uncertainty conditions.

The detection and characterization of pathologies is based on the interpretation of the alterations of the measurements due to the presence of the pathology. Other model uncertainties and system noises also alter these measurements. We can estimate the POD by the probability that the alteration of the measurement caused by the pathology is larger than that caused by the noise. If we label the alteration on the measurement readings caused by the pathology as the SIGNAL component, and the alteration generated by the noise as NOISE, the former definition can be formulated as (see Rus et al.[33]),

$$
\mathrm{POD}=P\left(\frac{|\mathrm{SIGNAL}|^{2}}{|\mathrm{NOISE}|^{2}}>1\right)
$$

Furthermore, three variables are be considered in the problem of maximizing the probability of detection (POD), the level of noise, denoted by $\sigma$, the location and extent of the pathologies, denoted by $\mathbf{p}$, and the cost functional that collects the effects of those in a scalar function $f$, as defined above.

We propose a new criterium of POD associated to multivariable pathologies amount with different references. It is define as follow.

$$
\begin{gathered}
\operatorname{RPOD}=\min _{\text {pat }_{p}} \operatorname{POD}\left(\text { pat }_{\mathrm{p}}\right) \\
\operatorname{POD}\left(\text { pat }_{\mathrm{p}}\right)=P\left(\frac{\mid\left.\operatorname{SigNAL}\left(\text { pat }_{p}\right)\right|^{2}}{\mid\left.\operatorname{NOISE}\left(\text { pat }_{p}\right)\right|^{2}}>1\right)
\end{gathered}
$$

where pat $=\left\{\Delta G^{c}=G^{c}-\tilde{G}^{c}, \Delta G^{d}=G^{d}-\tilde{G}^{d}\right\}, G$ is shear modulus, 
$\tilde{G}$ is reference measurement of shear modulus, and $c$ and $d$ are parameters relative to connective and dermic tissue respectively, $r$ is range of pathology and $p$ are parameters associated to model design.

From the definition of the simulated noise, the dependency of the variation of the measurement with increasing noise is also linear. These two considerations about linearity support the proposal that the measurements on a specimen with noise and with pathology can be expressed as Taylor series expansion centered at the case without noise and without pathology , and neglecting higher order terms (hot) than linear,

$$
\psi_{i}(p, \sigma)=\psi_{i}(0,0)+\underbrace{p \frac{\partial \psi_{i}}{\partial p}(0,0)}_{\text {SIGNAL }}+\underbrace{\sigma \frac{\partial \psi_{i}}{\partial \sigma}(0,0)}_{\text {NOISE }}+h o t
$$

where $i=1, \ldots, N_{i}$ are the measuring points. The first term on the right hand side is the measurement at point $i$ without noise nor pathology. The second term is the alteration of that measurement due to the presence of the pathology only, and is labeled SIGNAL, following the reasoning above. The third term is the alteration of the signal originated by the noise only (NOISE).

The second term of the Taylor series (equation 3.9) depends on the sensitivity of the measurements on the pathology, and can be approximated by finite differences,

$$
\frac{\partial \psi_{i}}{\partial p}\left(p_{0}, 0\right)=\psi_{i, p}\left(p_{0}, 0\right)=\frac{\psi_{i}\left(p_{0}+\Delta p, 0\right)-\psi_{i}\left(p_{0}-\Delta p, 0\right)}{2 \Delta p}
$$

where $p_{0} \rightarrow 0$ is a small pathology used to guarantee that the FEM captures the perturbations produced at small $\Delta p$ (since the case $p=0$ with no pathology needs to be computed with a topologically different mesh), in order to compute $\psi_{i, p}\left(p_{0}, 0\right) \approx \psi_{i, p}(0,0)$. In addition, a central difference scheme, which yields an error of the order $O\left(\Delta p^{2}\right)$, becomes available. Since 
the noise component is linear by definition, a forward difference scheme is adopted, whose $O(\Delta \sigma)$ error is sufficient.

Some authors [36] propose that the parameters $\Delta p$ and $\Delta \sigma$ should be two orders of magnitude smaller than the values at which the derivative should be computed. However, an estimation of these parameters is studied. It shows $\psi_{i, p}(0,0)$ and $\psi_{i, \sigma}(0,0)$ versus $\Delta p$ and $\Delta \sigma$, respectively, for a pathology at the center of the bridge deck. $\Delta p=\Delta \sigma=10^{-2}$ is shown to produce a stable value of the derivative for the case of the single measurement represented, but the same result is obtained for all 18 measuring points.

The third term of the Taylor series (equation 3.9) can be directly derivated if the equation 3.6 is assumed,

$$
\frac{\partial \psi_{i}}{\partial \sigma}=\xi_{i} \operatorname{RMS}\left(\psi_{i}^{\mathrm{FEM}}\right)=\xi_{i} \mathrm{RMS}
$$

Equations (3.9), (3.11) and the relationship $\left|Y_{i}\right|^{2}=\frac{1}{m} \sum_{i=1}^{m} Y_{i}^{2}$, can be combined into (3.6) to obtain,

$$
\mathrm{POD}=P\left(\frac{p^{2} \frac{1}{N_{i}} \sum_{i=1}^{N_{i}}\left(\psi_{i, p}(0,0)\right)^{2}}{\sigma^{2} \mathrm{RMS}^{2} \frac{1}{N_{i}} \sum_{i=1}^{N_{i}} \xi_{i}^{2}}>1\right)=P\left(p^{2}>\frac{\operatorname{RMS}^{2} \sigma^{2} \sum_{i=1}^{N_{i}} \xi_{i}^{2}}{\sum_{i=1}^{N_{i}}\left(\psi_{i, p}(0,0)\right)^{2}}\right)
$$

If the noise generator $\xi_{i}$ is a random variable, the POD is a probability of the stochastic variable $p^{2}$, described by the cumulative probability density function $F$,

$$
\mathrm{POD}=F\left(\frac{\mathrm{RMS}^{2} \sigma^{2} \sum_{i=1}^{N_{i}} \xi_{i}^{2}}{S_{p}}\right)
$$


Using Monte Carlo techniques and error propagation theory the noise in the measurement points can be concluded to follow a normal distribution (Rus et al[33]). Assuming this distribution, the squared sum of the noise $\xi_{i}$ is known to follow a Chi-square distribution, since $\sum_{i=1}^{N_{i}} \xi_{i}^{2} \longrightarrow \chi_{N_{i}}^{2}$ (e.g. [37]). The parameter of the Chi-square distribution is the number of degrees of freedom $N_{i}$, which in this case is the number of measurement points. In the case that $N_{i}>10$, the Chi-square distribution can be approximated by a Gaussian or normal $N$ distribution $\chi^{2}\left(N_{i}\right) \approx N\left(N_{i}-2 / 3, \sqrt{2 N_{i}}\right)$ with mean $N_{i}-2 / 3$ and standard deviation $\sqrt{2 N_{i}}$. This approximation in (3.13) yields,

$$
p^{2} \longrightarrow N\left[\frac{\mathrm{RMS}^{2} \sigma^{2}\left(N_{i}-2 / 3\right)}{S_{p}}, \frac{\mathrm{RMS}^{2} \sigma^{2} \sqrt{2 N_{i}}}{S_{p}}\right]
$$

Since $F(x)=\int_{-\infty}^{x} f(y) \mathrm{d} y$ is the cumulative of the normal probability density function $f$, whose inverse is $x=G(F(x))$, the useful pathology area to noise ratio $p / \sigma$ can be expressed from (3.14) given a POD level as,

$$
\frac{p}{\sigma}=\sqrt{\frac{\operatorname{RMS}^{2}\left(N_{i}-2 / 3\right)}{S_{p}}\left(1+G[\mathrm{POD}] \frac{\sqrt{2 N_{i}}}{N_{i}-2 / 3}\right)}
$$

Note that the analytical expression (3.14) is only valid for noise with normal distribution at the measurement points.

To maximize the POD is necessary to define a pessimistic approach that is formulated below. It consists in minimizing the minimum vector of the POD pathologies, applied to a range of values for model parameters. POD compound will be appreciate in effects of POD in next sections.

$$
\mathrm{RPOD}=\min _{p} \min _{\text {pat }_{p}} \mathrm{POD}\left(\text { pat }_{p}\right)
$$




\subsection{Numerical Results}

The purpose of the numerical results is to obtain conclusions about which experimental design is better in characterizing mechanical tissue properties. To the latter end, three independent criteria are evaluated numerically: The effects of the excitation and driving frequency combination on (i) the measurements, (ii) the cost function and (iii) the POD, are studied for a set of configurations. The scope is to extract some a priori thumb rules that allow to select those with a more accessible minimum in the cost function, and guarantee satisfactory results for a minimization algorithm.

\subsubsection{Excitation, model displacement}

Consider the specimen described above.

A sample of resulting measurements is shown in Figure 3.2.

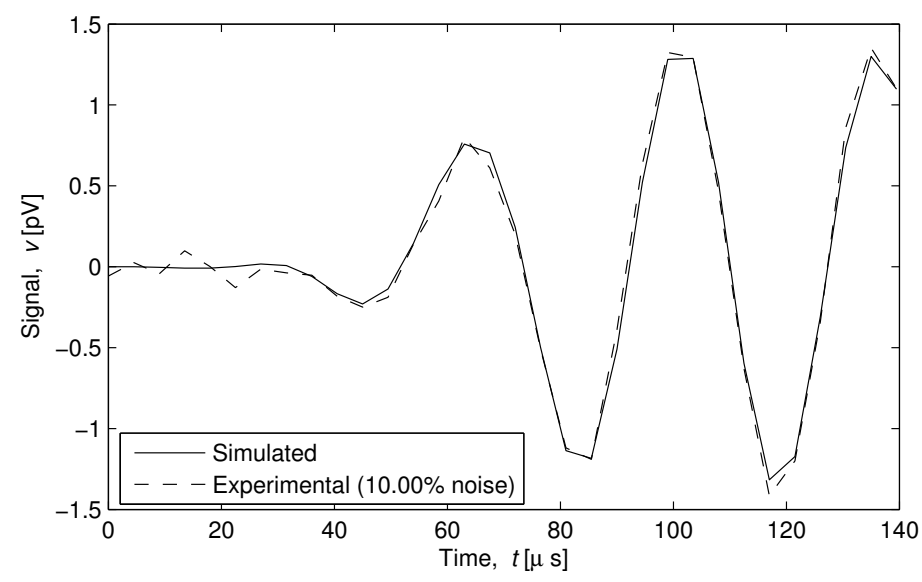

Figure 3.2: Simulated measurements for model design, noise $0.10 \%$

The results of the simulated signal with a $10 \%$ of noise are consistent with the response in the FEM model described in the previous chapter. 


\subsubsection{Effects on cost functional}

The shape of the cost function provides another subjective way to evaluate the sensitivity of the numerically predicted signals, based on the following criteria:

- The existence of local or global minima affects the convergence of the search algorithm.

- Steep minima are better than those providing soft valleys, due to algorithm convergence performance.

- Valleys that present shapes close to circular are considered as an indicator of uncoupled mechanical properties of soft tissue parameters.

Figure 3.3 shows a slice of the multidimensional cost function as functions of the parameters $G_{d}$ and $G_{c}$ for configuration of the model.

\subsubsection{Effects on POD}

The aforementioned criteria is aimed at evaluate the local behavior of the cost functional, regardless of the noise effects. Maximizing the POD enables to find the smallest pathology given the largest noise levels, independently of the robustness of the convergence of the search. Figure 3.4 shows an example of the POD estimation for one excitation configuration for increasing pathological values, whereas the dependency of the POD on the pathology extent is illustrated for a fixed noise level that amounts to $0.1 \%$.

\subsubsection{POD optimization}

Once identified the effects of the POD, the optimization is calculated to demonstrate if the POD improved by optimal design. 

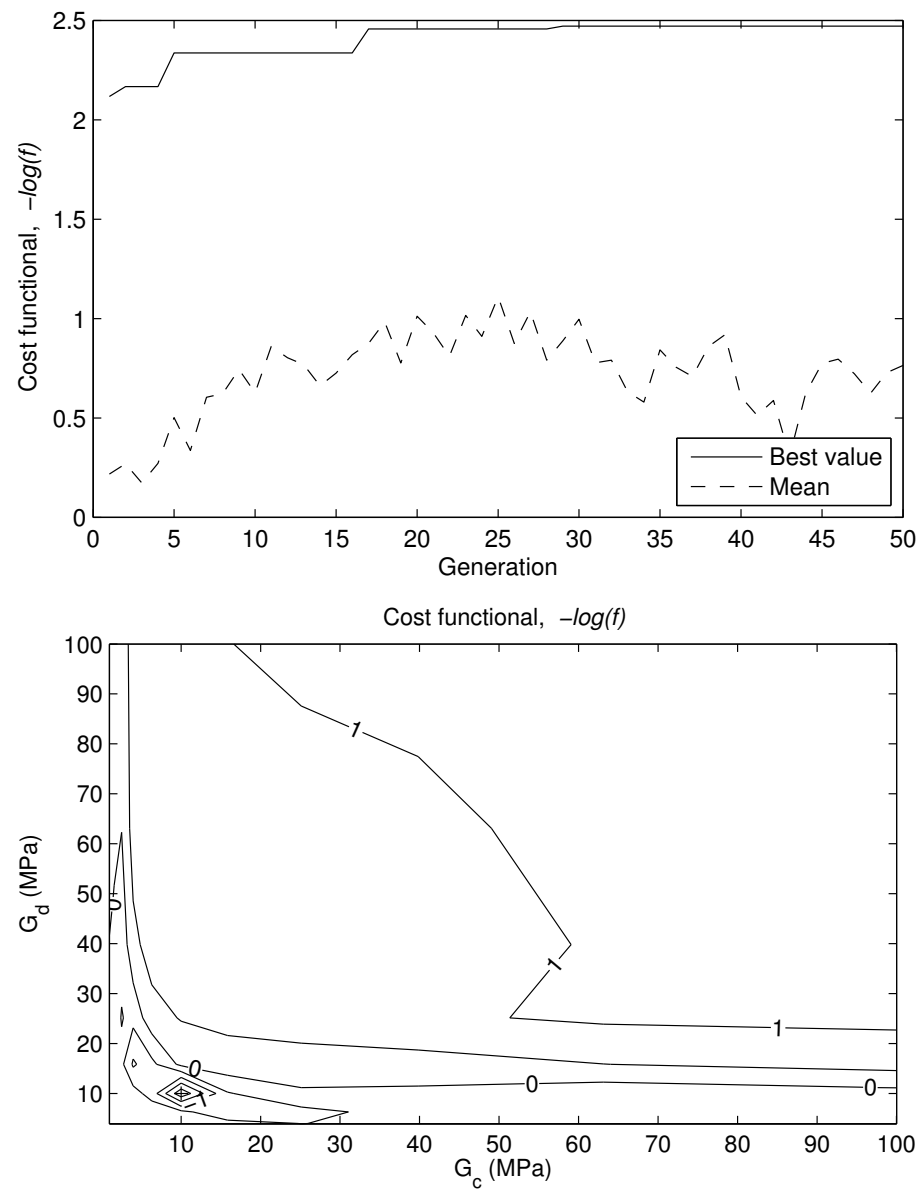

Figure 3.3: Cost functional as a function of the parameters with reference $G_{d}$ and $G_{c}$

The first four graphs show the regions of maximum POD over the 8 parameters of the model, the fifth graph relates the genetic algorithms search with a population of 20 individuals and 50 mutations and the best value and the last graph shows the optimized parameters for the best POD . 

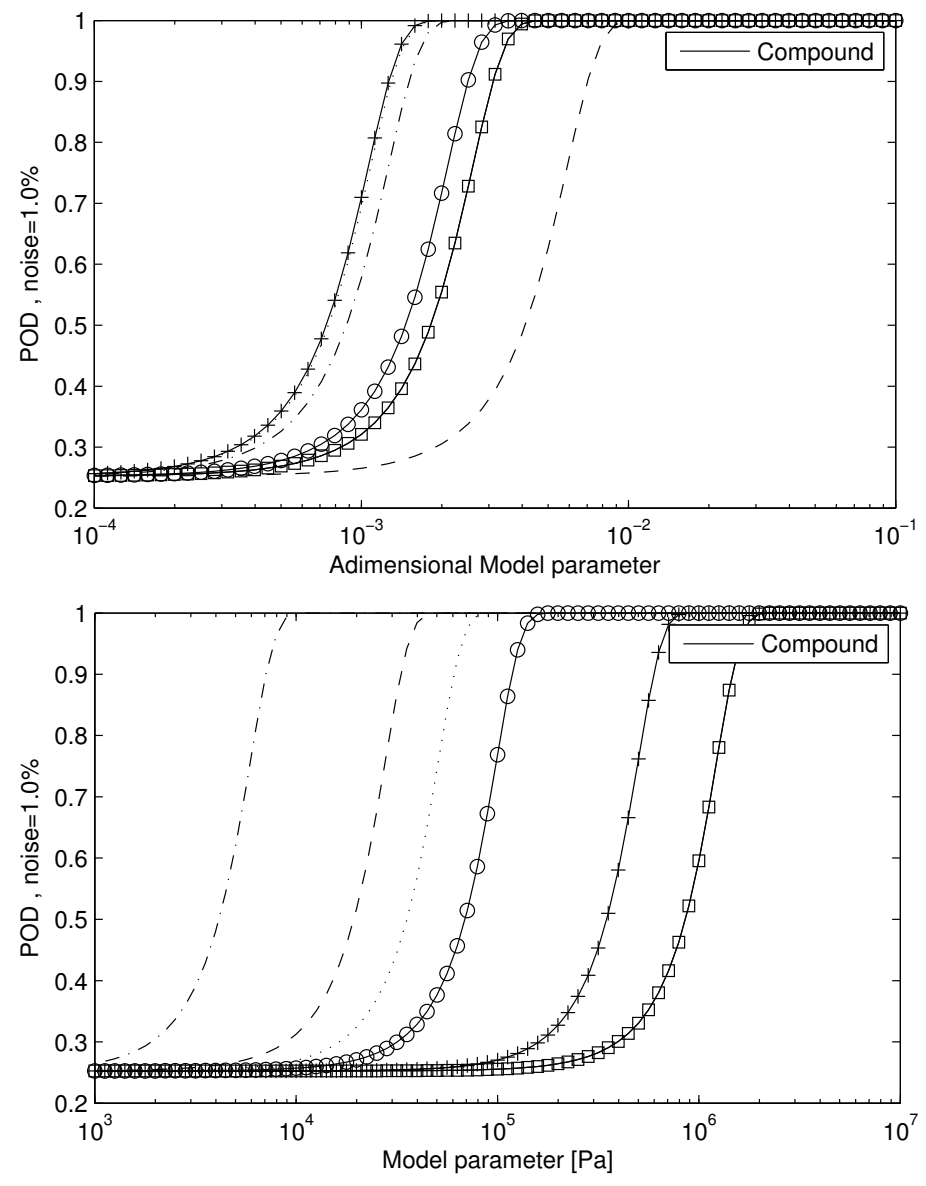

Figure 3.4: Dependency of the POD on the pathology amount.

\subsection{Conclusions and impact}

\subsubsection{Conclusions}

In this chapter, we have developed a strategy based on the inverse problem to optimize the model developed in the previous chapter.

First we define a cost function as the difference between the experimental and theoretical signals and other statistical tools. Then, we define the POD (Probability of detection) as the probability that the signal is greater than the noise through a Taylor series expansion and a finite difference scheme.

After reformulated this concept to the case that concerns us, the better prediction of probability of detection for preterm birth. Through three references in different stages of gestation by G modulus, 300, 3000 and 30000, RPODD is defined as robust probability of detection with a pessimistic ap- 

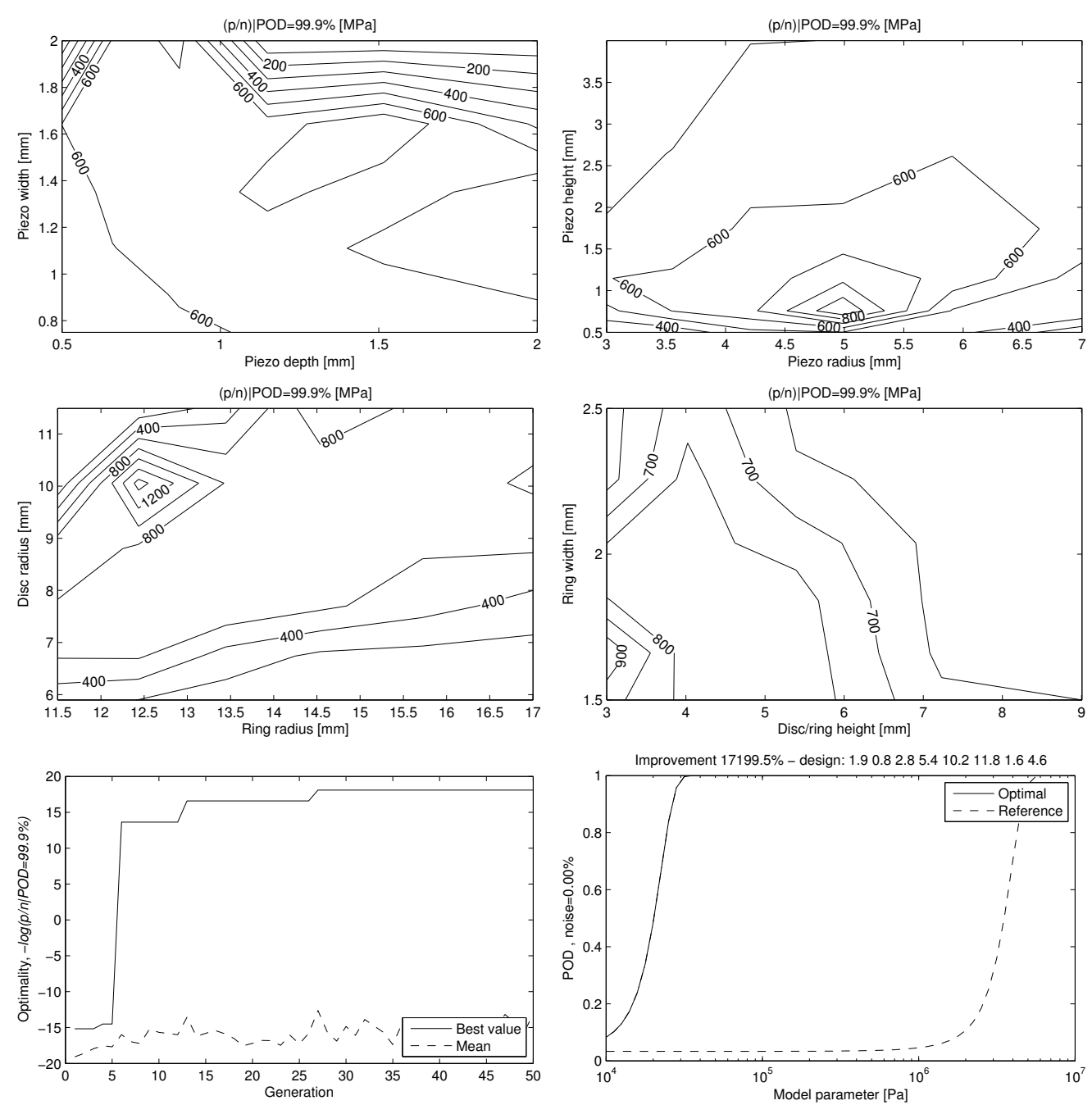

Figure 3.5: POD optimization

proach, the minimum of the minimum value of POD vector of components associated with pathological range of values in parameters $\mathrm{p}$ of the model.

Finally algorithms are developed on the RPOD, obtained best results optimized design through this criterion as shown above graphs.

\subsubsection{Impact}

A parametric study is performed in order to assess how sensor design parameters and vehicle characteristics control the identifiability. This serves as design criteria to guarantee the feasibility of robust monitoring.

Improvements in the treatment of preterm infants have helped to greatly improve their survival. However, these infants remain vulnerable to many 
complications, including respiratory, gastrointestinal, immune system, central nervous system, hearing, and vision problems. The effects may last well beyond childhood and include cerebral palsy, mental retardation, visual and hearing impairments, behavior and social-emotional concerns, mother suffering, learning difficulties, and poor health and growth[38]. Babies born before 32 weeks have the greatest risk for death and poor health outcomes, however infants born between 32 and 36 weeks, which make up the greatest number of preterm births, are still at higher risk for health and developmental problems compared to those infants born full term. The annual societal economic burden associated with preterm birth, that affects $8.1-12.7 \%$ of all births in Europe[39] (and increasing over the last 40 years[40]) was at a minimum $\$ 26$ billion in 2005 in the US, or $\$ 51600$ per infant born preterm [41]. Nearly two thirds of this cost was for medical care. There is currently no test that can accurately predict a preterm birth with sufficient anticipation to allow effective actions, and little is known about how a preterm birth can be prevented. Treatment has been primarily focused on slowing contractions, which has helped reduce the rates of mortality and morbidity. Yet therapies and interventions for the prediction and the prevention of preterm birth are still greatly needed.

Outside the field of obstetrics and gynecology, which is the first applied target on which this project focuses, a technology for diagnosis based on mechanical properties has a vast potential for other tissues, like breast or prostate tumors or liver disorders. As an example, breast cancer screening faces an important limitation due to the ionizing nature of the established mammography. This has motivated several scientists to propose various ultrasound-based diagnosis techniques. An example of the success of quantitative sonography is given by Yonetsu[25], who show that quantitative techniques provide effective results in direct diagnosis of differentiation in 
benign or malignant tumors and their type. Since nodules show up very strongly by a drastic consistency change, the proposed diagnosis technology based on mechanical properties could have a strong impact on several health issues and become an excellent candidate for future research.

Another potential is to create and publish a database of in vivo mechanical properties of various tissues and organs, which is currently unavailable and much needed by the biomechanics scientific community. These examples envisage the scientific and technological long term horizon of the idea behind this project. 


\section{Appendix A}

\section{D piezoelectric laminate}

\section{validation}

\section{A.1 Analytic 1D validation}

Consider the wave propagation in a structure with a piezoelectric layer of thickness $h$. The piezoelectric relations are assumed linear and quasistationary electric field is considered.

In rectangular Cartesian coordinates, the stress equations of motion are given by

$$
\sum_{j=1}^{3} T_{i j, j}=\rho \ddot{u}_{i}
$$

for $i=1,2,3$ where $T_{i j}$ is the stress tensor, $u_{i}$ the mechanical displacement, $\rho$ the mass density, and subscript " $j "$ indicates differentiation with respect to $x_{j}$. The electric displacement $D_{i}$ satisfies Maxwell's equation,

$$
\sum_{i=1}^{3} D_{i, i}=0
$$

The electric field $E_{i}$ (for $i=1,2,3$ ) is related to the electric potential $\phi$ 
by

$$
E_{i}=-\phi_{i}
$$

With the constitutive equations:

$$
\begin{aligned}
S & =S_{E} T+d^{t} E \\
D & =d T+\epsilon_{T} E
\end{aligned}
$$

where $\mathrm{S}$ is Strain, $S_{E}, d^{t}, d$ and $\epsilon_{T}$ are respectly.

Using maxwell equation and dynamic equilibrum we have:

$$
\begin{gathered}
\nabla\left(S_{E} T+d^{t} E\right)=\nabla S \\
S_{E} \nabla T+d^{t} \nabla E=\nabla \nabla^{S} u \\
S_{E} \rho \ddot{u}+d^{t} \nabla E=\nabla \nabla^{S} u
\end{gathered}
$$

On the other side,

$$
\begin{gathered}
\nabla\left(d T+\epsilon_{T} E\right)=0 \\
d \nabla T+\epsilon_{T} \nabla E=0 \\
d \rho \ddot{u}+\epsilon_{T} \nabla E=0 \\
d \nabla T=-\epsilon_{T} \nabla E \\
-\epsilon_{T}^{-1} d \nabla T=\nabla E
\end{gathered}
$$


Thus, substituting in equation (8):

$$
S_{E} \rho \ddot{u}-d^{t} \epsilon_{T}^{-1} d \rho \ddot{u}=\nabla \nabla^{S} u
$$

And,

$$
\triangle \phi=\epsilon_{T}^{-1} d \rho \ddot{u}
$$

Piezoelectric coefficients:

$K=\left[\begin{array}{cccccc}0.1271 e-06 & -0.0445 e-6 & -0.0560 e-6 & 0 & 0 & 0 \\ -0.0445 e-6 & 0.1271 e-6 & -0.0560 e-6 & 0 & 0 & 0 \\ -0.0560 e-6 & -0.0560 e-6 & 0.1457 e-6 & 0 & 0 & 0 \\ 0 & 0 & 0 & 0.3681 e-6 & 0 & 0 \\ 0 & 0 & 0 & 0 & 0.3681 e-6 & 0 \\ 0 & 0 & 0 & 0 & 0 & 0.3433 e-6\end{array}\right]$

where:

$$
S_{E} \rho-d^{t} \epsilon_{T}^{-1} d \rho=K
$$

and,

$$
L=\left[\begin{array}{cccccc}
0 & 0 & 0 & 0 & 0.2616 e-8 & 0 \\
0 & 0 & 0 & 0.2616 e-8 & 0 & 0 \\
-0.0780 e-8 & -0.0780 e-8 & 0.1705 e-8 & 0 & 0 & 0
\end{array}\right]
$$

where:

$$
\epsilon_{T}^{-1} d \rho=L
$$

The constitutive equations are different for different dependent on the types of piezoelectric materials considered. In this paper, the piezoelectric 
material of hexagon crystal structure, class $0.05 \mathrm{~mm}$, is employed. For other types of piezoelectric material, the constitutive equations should be changed accordingly. Assuming the six-fold axes of the piezoelectric material parallel to the $x_{3}$-direction, its constitutive equations can be expressed in the form:

$$
\begin{aligned}
& S_{11}=S_{11}^{E} T_{11}+S_{12}^{E} T_{22}+S_{13}^{E} T_{33}-d_{31}^{T} E_{3}, \\
& S_{22}=S_{12}^{E} T_{11}+S_{11}^{E} T_{22}+S_{13}^{E} T_{33}-d_{31}^{T} E_{3}, \\
& S_{33}=S_{13}^{E} T_{11}+S_{13}^{E} T_{22}+S_{33}^{E} T_{33}-d_{33}^{T} E_{3}, \\
& S_{23}=S_{44}^{E} T_{23}-d_{15}^{T} E_{2}, \\
& S_{13}=S_{44}^{E} T_{13}-d_{15}^{T} E_{1}, \\
& S_{12}=S_{66}^{E} T_{12}=0.5\left(S_{11}^{E}-S_{12}^{E}\right) T_{12}, \\
& D_{1}=d_{15} T_{13}+\epsilon_{11}^{T} E_{1}, \\
& D_{2}=d_{15} T_{23}+\epsilon_{11}^{T} E_{2}, \\
& D_{3}=d_{31} T_{11}+d_{31} T_{22}+d_{33} T_{33}+\epsilon_{33}^{T} E_{3},
\end{aligned}
$$

where the coefficients $d, S^{E}$ and $\epsilon$ are the elastic, piezoelectric and dielectric constants, and the strain components are defined as:

$$
\begin{array}{r}
S_{11}=u_{1,1} S_{2,2}=u_{2,2} S_{33}=u_{3,3} \\
S_{23}=u_{2,3}+u_{3,2} S_{13}=u_{3,1}+u_{1,3} S_{12}=u_{2,1}+u_{1,2} .
\end{array}
$$

Restricted to 3-direction:

$$
\begin{aligned}
u_{3,3} & =S_{33}^{E} T_{33}-d_{31}^{T} E_{3} \\
D_{3} & =d_{33} T_{33}+\epsilon_{33}^{T} E_{3}
\end{aligned}
$$

Derivating two expressions, results

$$
\begin{aligned}
u_{3,33} & =S_{33}^{E} T_{33,3}-d_{31}^{T} E_{3,3} \\
0 & =d_{33} T_{33,3}+\epsilon_{33}^{T} E_{3,3}
\end{aligned}
$$




$$
\begin{aligned}
u_{3,33} & =S_{33}^{E} \rho \ddot{u_{3}}-d_{31}^{T} E_{3,3} \\
0 & =d_{33} \rho \ddot{u_{3}}+\epsilon_{33}^{T} E_{3,3}
\end{aligned}
$$

Thus,

$$
\begin{gathered}
u_{3,33}=S_{33}^{E} \rho \ddot{u_{3}}-d_{31}^{T} E_{3,3} \\
E_{3,3}=-d_{33} \rho \ddot{u_{3}} / \epsilon_{33}^{T} \\
u_{3,33}=S_{33}^{E} \rho \ddot{u_{3}}+d_{31}^{T} d_{33} \rho \ddot{u_{3}} / \epsilon_{33}^{T} \\
E_{3,3}=-d_{33} \rho \ddot{u_{3}} / \epsilon_{33}^{T} \\
u_{3,33}=\underbrace{\left(S_{33}^{E}+d_{31}^{T} d_{33} / \epsilon_{33}^{T}\right) \rho}_{K_{33}} \ddot{u}_{3} \\
E_{3,3}=-d_{33} \rho \ddot{u}_{3} / \epsilon_{33}^{T}
\end{gathered}
$$

And the we have two systems of PDE:

$$
\begin{aligned}
\frac{\partial^{2} u}{\partial x^{2}} & =K_{33} \frac{\partial^{2} u}{\partial t^{2}} \\
\frac{\partial \phi}{\partial x} & =\underbrace{d_{33} \rho / \epsilon_{33}^{T}}_{L_{33}} \frac{\partial^{2} u}{\partial t^{2}}
\end{aligned}
$$

Boundary conditions

$$
\begin{aligned}
u(0, t) & =f(t) \\
\left.u_{3,3}(x, t)\right|_{x=L} & =S_{33}^{E} T_{33}^{i m p}-d_{33}^{T} E_{3}^{i m p} \\
u(x, t) & =\frac{1}{T} \sum_{-\infty}^{\infty} \tilde{u}\left(x, \omega_{n}\right) e^{2 \pi \omega_{n} t}
\end{aligned}
$$


being $\omega_{n}=\frac{n}{T}$

Equation can be rewritten as,

$$
\frac{1}{K_{33}} \tilde{u}^{(n)^{\prime \prime}}(x, \omega)+\omega_{n}^{2} \tilde{u}^{(n)}(x, \omega)=0
$$

Therefore, a general harmonic solution to equation 48 can be stated as,

$$
\tilde{u}^{(n)}(x, \omega)=\tilde{u}^{(n), f}(x, \omega)+\tilde{u}^{(n,) b}(x, \omega)=A^{(n)} \cdot e^{-i k_{n} x}+B^{(n)} \cdot e^{-i k_{n} x}
$$

where $\tilde{u}^{(n), f}(x, \omega)$ and $\tilde{u}^{(n), b}(x, \omega)$ stand for the forward- and backwardpropagating parts of the linear displacement, respectively. The upper index (n) denotes the order of the considered harmonic component, with respect to the natural frequency $\omega . k_{n}$ denotes the complex wave number and results from, $k_{n}=\frac{\omega_{n}}{c_{p}}=n k=n \bar{k}(1-i \alpha), n \in \mathbb{N}^{*}$ where $\bar{k}$ and $\alpha$ respectively denote the real part of the wave number and the Rayleigh damping coefficient [42].

To complete the analytical formulation of the problem, first we developed for the problem amplitude 1D static and then frequency for the 1D dynamic problem.

- Amplitude for static 1D case:

$$
\begin{gathered}
T=0 \\
E=100 \mathrm{~V} \\
S_{33}=u_{3,3}=\frac{\partial u_{3}}{\partial x_{3}}
\end{gathered}
$$


Then,

$$
u_{3}(t)=\int_{0}^{L} S_{33} d x_{3}=S_{33} \int_{0}^{L} d x_{3} L S_{33}
$$

Using (A.4) we conclude that,

$$
S=S_{E} \underbrace{T}_{=0}+d^{t} E
$$

- Speed $v$ for the $1 \mathrm{D}$ case dynamic steady

$$
\begin{aligned}
& T=0 \\
& E=100 \mathrm{~V}
\end{aligned}
$$

Where $\mathrm{E}$ is an electric pulse.

Given (A.47):

$$
u_{3}(x, t)=\operatorname{Re}\left[u_{3}^{a m p l} e^{i\left(\omega\left(t-\frac{x}{c}\right)\right)}\right]=u_{3}^{a m p l} \sin \left(\omega\left(t-\frac{x}{c}\right)\right)
$$

Considering equation (A.43) we deduce that:

$$
\begin{gathered}
u_{3, x x}=K_{33} u_{3, t t} \\
\left(\frac{\omega}{c}\right)^{2} u_{3}^{a m p l} \sin \left(\omega\left(t-\frac{x}{c}\right)\right)=K_{33} \omega^{2} u_{3}^{a m p l} \sin \left(\omega\left(t-\frac{x}{c}\right)\right)
\end{gathered}
$$

Whereby the speed is as follows:

$$
c=\sqrt{\frac{1}{K_{33}}}
$$




\section{A.2 Results}

To validate that the piezoelectric element introduced in the torque sensor as a 3D laminate had the desire effect, a finite elements model was calculated whose displacements response coincided with the results of the analytical model based on the constitutive equations of piezoelectric. Following figures shows that FEAP software response coincides with that expected in the analytical formulation.
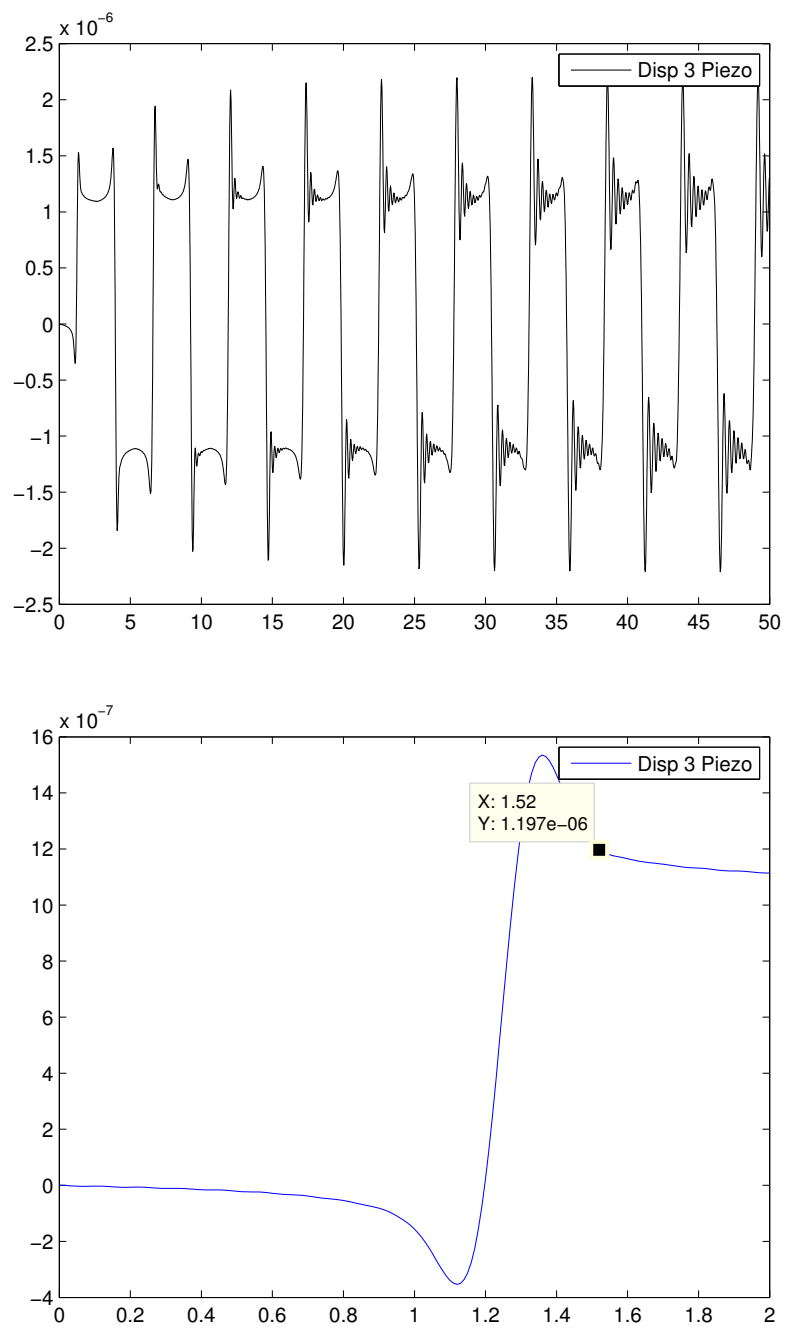

Figure A.1: Dynamic 1D problem

Using dynamic displacement response, is possible to deduct the time that begins the movement of the transient part and find the wave speed for 
comparison with the analytical model.
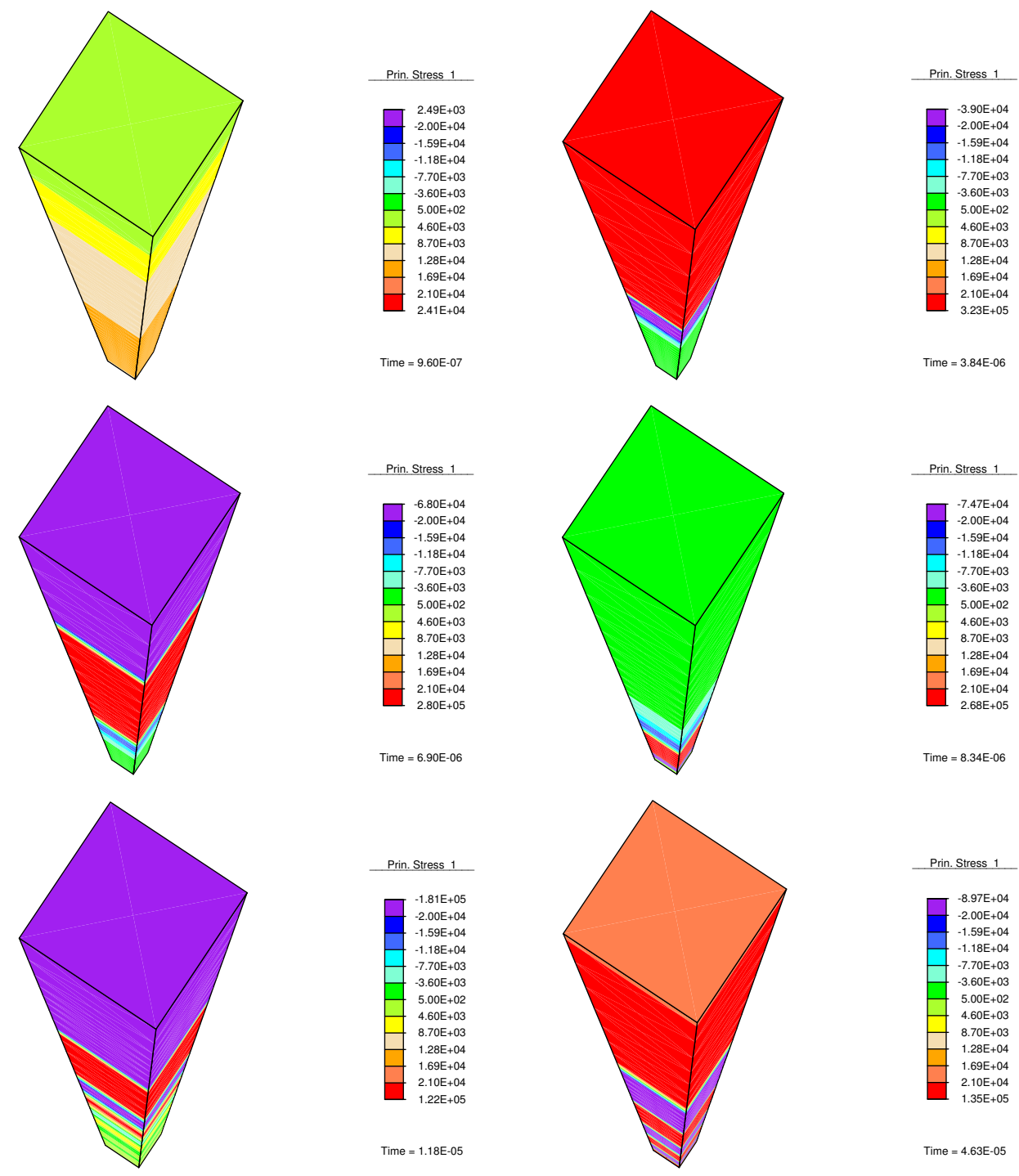

Figure A.2: Different states of piezoelectric 3D element

The result of FEAP developed with FEM gives a similar result to analytically deduced. The following table shows the outcomes in speed and the degree of error being validated 3D piezo material for implementing the calculation of the torque sensor design. 


\begin{tabular}{c|c}
\hline \hline Analytical speed & $8.5 \mathrm{e} 3[\mathrm{~m} / \mathrm{s}]$ \\
FEAP speed & $8.3 \mathrm{e} 3[\mathrm{~m} / \mathrm{s}]$ \\
Speed percentage of error & $1.19 \%$ \\
Analytical displacement voltage & $3.74 \mathrm{e}-8[\mathrm{~V}]$ \\
FEAP displacement voltage & $1.482 \mathrm{e}-8[\mathrm{~V}]$ \\
Voltage percentage of error & $25.1 \%$ \\
\hline \hline
\end{tabular}

Table A.1: Validation 1D piezo material 


\section{Appendix B}

\section{Torsion sensor model algorithms}

This appendix provides a summary of the algorithms developed in calculating the properties of the piezoelectric material used in the finite element model, the model design through which the sensitivities were analyzed, and the analytical simplified design codes for validation of the results.

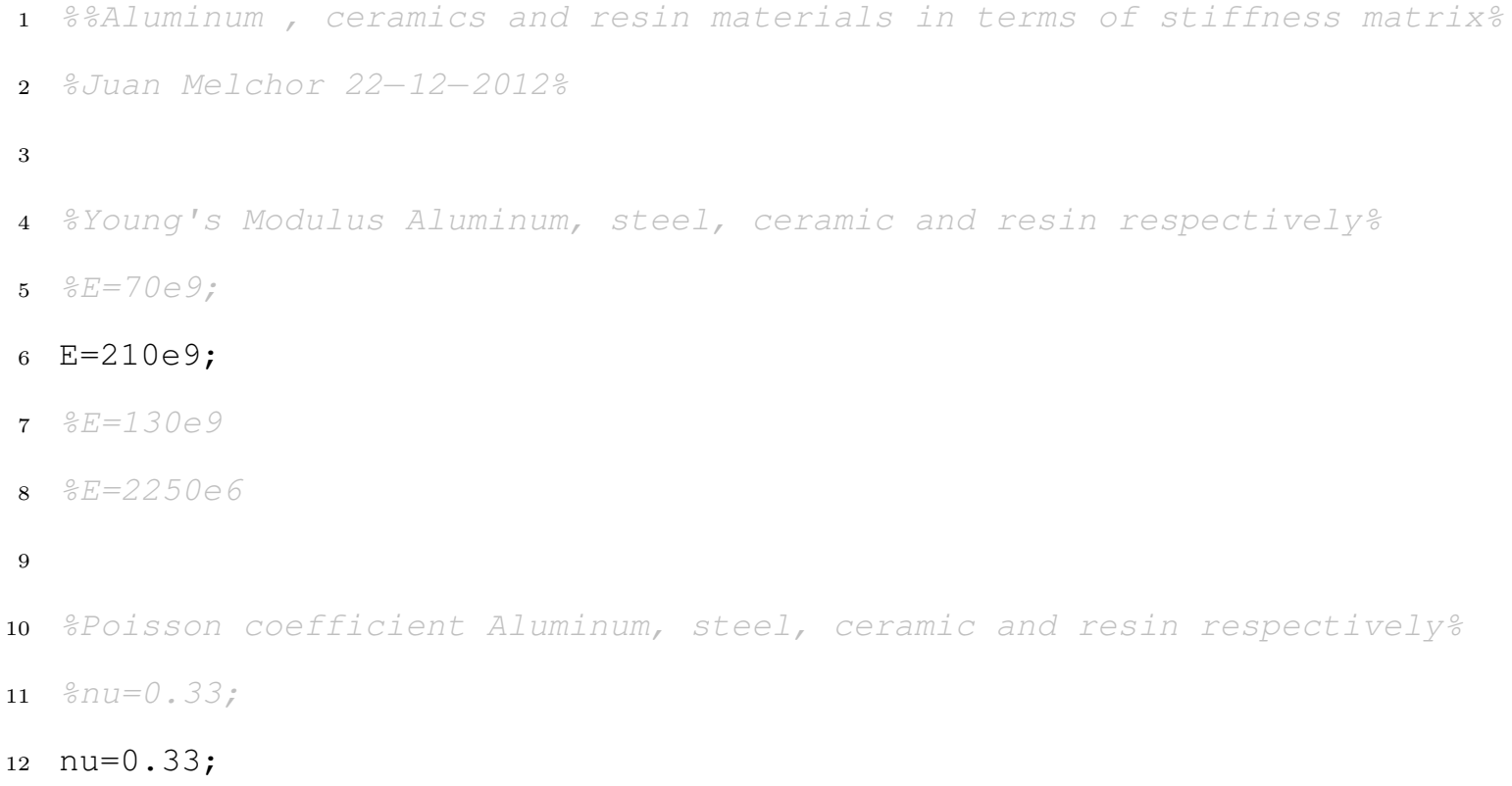




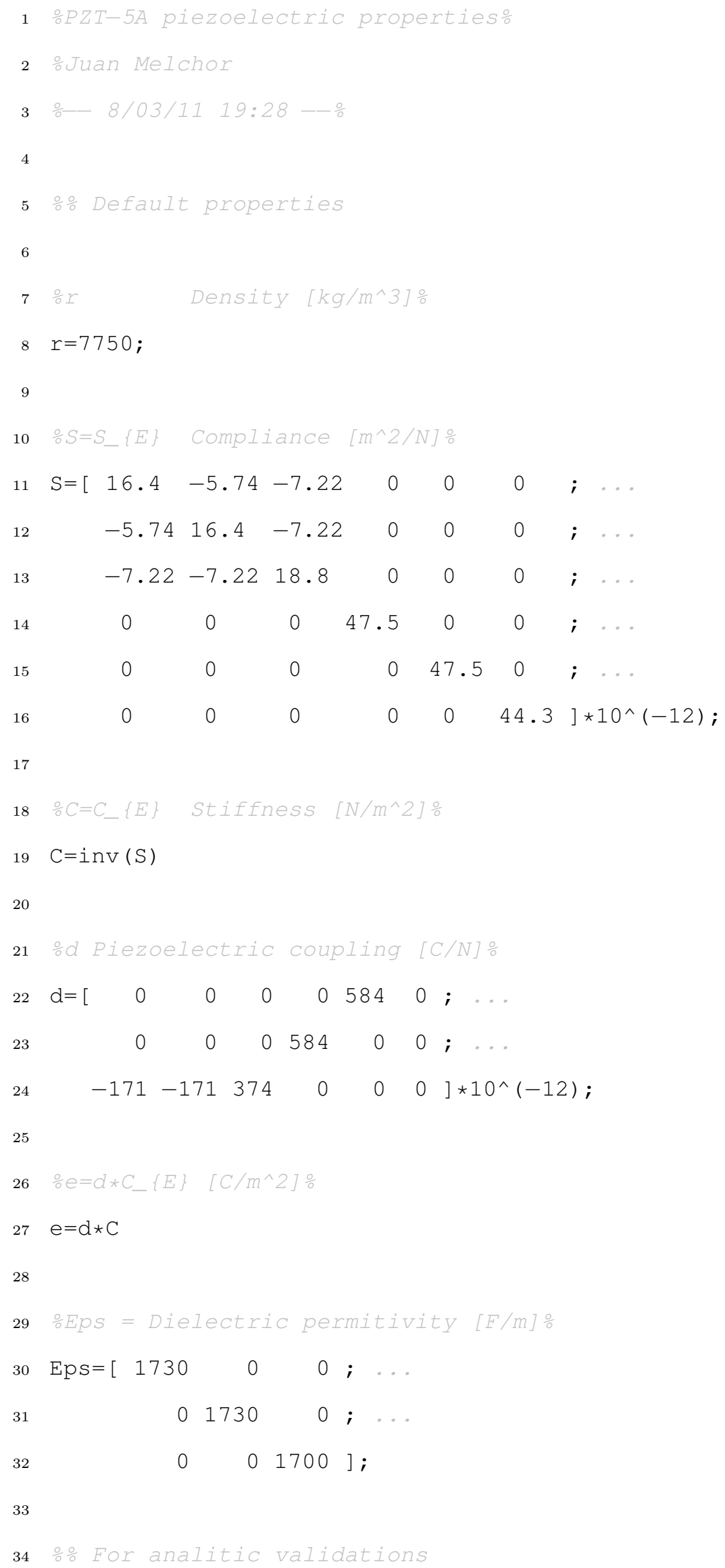


35

36

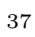

38

39

40

$41 \mathrm{k}=\mathrm{inv}(\mathrm{Eps}) \star \mathrm{d} * r$;

42

43

44

45

46

47

48

60

61

62

63

64

65

66

68

c3=inv (c2) ;

$l_{y}=\left[\begin{array}{lllll}1 & 0 & 0 & ;\end{array}\right.$

$001 ; \ldots$

$\left.\begin{array}{lll}0 & 1 & 0\end{array}\right]$

$\left.\begin{array}{llllll}0 & 0 & 0 & 0 & 1 & 0\end{array}\right]$;

alpha=pi $/ 4$;

$\mathrm{CS}=\cos (\mathrm{alpha})$;

$\mathrm{SN}=\sin (\mathrm{alpha})$;

$l \mathrm{z}=\left[\begin{array}{lll}\mathrm{CS}-\mathrm{SN} & 0\end{array}\right.$

SN CS 0 ;

o 0 1];

onalytical validation coefficients in displacement 3x3x3x3응

$c 2=S \star r-d^{\prime} \star i n v(E p s) \star d * r$

ocoeficients of analytical validation 3x3x3응

응 Material rotation

응 Exchanging $Z\left\langle->X\right.$ (equivalent to $90^{\circ}$ on $Y$ ) + rotate pi/ 4 around $Z$

응 Rotation matrices $I$ of $i j[6 * 6]$ and $I$ i [3*3]

$L y=\left[\begin{array}{llllllll}1 & 0 & 0 & 0 & 0 & 0 & ; & \ldots\end{array}\right.$

$\begin{array}{llllllllll}0 & 0 & 1 & 0 & 0 & 0 & ;\end{array}$

$\begin{array}{lllllllllll}0 & 1 & 0 & 0 & 0 & 0 & ;\end{array}$

$\begin{array}{lllllllllll}0 & 0 & 0 & 1 & 0 & 0 & ;\end{array}$

$\begin{array}{llllllllll}0 & 0 & 0 & 0 & 0 & 1 & \ldots\end{array}$

$\mathrm{Lz}=\left[\begin{array}{llllll}\mathrm{CS}^{\wedge} 2 & \mathrm{SN}^{\wedge} 2 & 0 & 0 & 0 & -2 * \mathrm{CS} * \mathrm{SN} ;\end{array}\right.$

$\begin{array}{llllll}\mathrm{SN}^{\wedge} 2 & \mathrm{CS}^{\wedge} 2 & 0 & 0 & 0 & 2 * \mathrm{CS} * \mathrm{SN} ; \ldots\end{array}$

$\begin{array}{llllllll}0 & 0 & 1 & 0 & 0 & 0 & ;\end{array}$

$\begin{array}{llllll}0 & 0 & 0 & C S-S N & 0 & ;\end{array}$

$\begin{array}{lllllll}0 & 0 & 0 & \text { SN } & C S & 0\end{array}$

$\left.\mathrm{CS} * \mathrm{SN}-\mathrm{CS} * \mathrm{SN} \quad 0 \quad 0 \quad 0-1+2 * \mathrm{CS}^{\wedge} 2\right]$;

$\mathrm{LZ}=\left[\begin{array}{llllll}\mathrm{CS}^{\wedge} 2 & \mathrm{SN}^{\wedge} 2 & 0 & 0 & 0 & 2 * \mathrm{CS} * \mathrm{SN} ; \ldots\end{array}\right.$

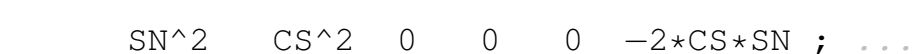




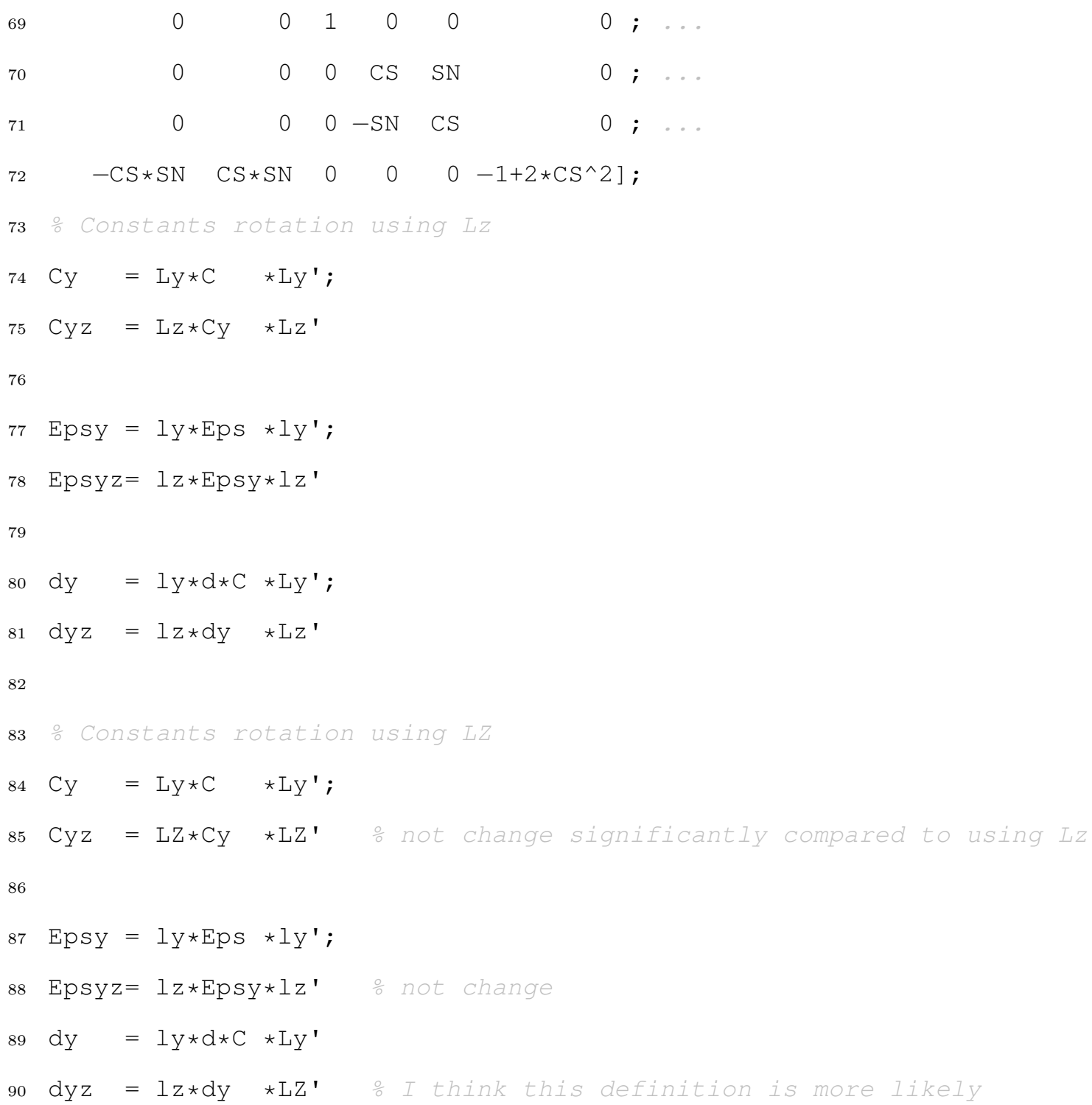




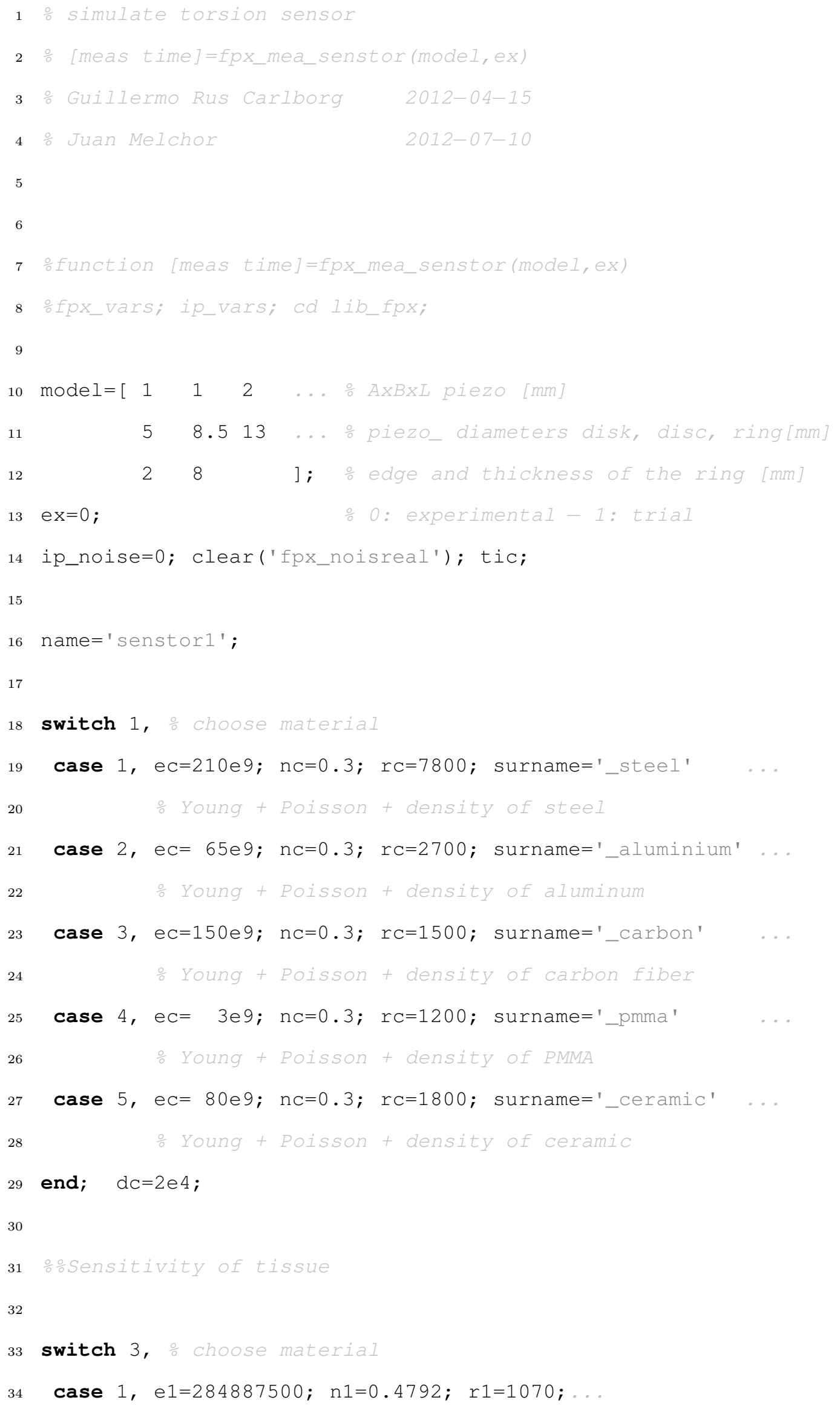


41

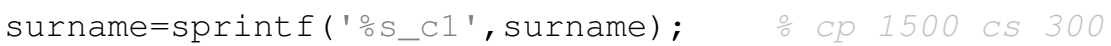

case $2, e 1=1.2763 e 8 ; n 1=0.4910 ; r 1=1070 ;$ surname=sprintf ('s_c2', surname); $\frac{\circ}{0}$ cp 1500 cs 200

case $3, e 1=282480000 ; n 1=0.4667 ; \quad r 1=1070$; surname=sprintf ('을', surname); $\quad \frac{\circ}{0}$ cp 1200 cs 300

\section{end;}

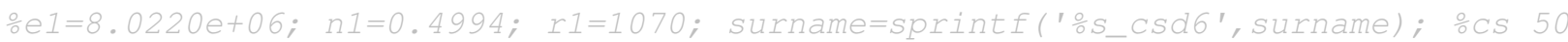

응 sitch cou (), ovarying in dermal tissue cs

case $1, e 1=1.2839 e+06 ; n 1=0.4999 ; \quad r 1=1070 ;$

...surname=sprintf ('\%s csdI', surname); \%cs 20

동 $2, e 1=3.2025 e+07 ; n 1=0.4965 ; \quad r 1=1070 ;$

... surname=sprintf ('\%s_csd2', surname); \%cs 100

aㅗ $\quad$ case,$e 1=1.2718 e+08 ; n 1=0.4857 ; \quad r 1=1070 ;$

...surname=sprintf('\%s_csd3', surname); \%cs 200

동 4, $\quad e 1=282480000 ; \quad n 1=0.4667 ; \quad r 1=1070 ;$

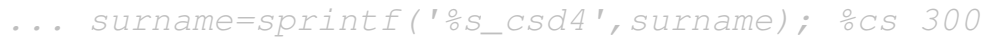

동 $\quad$ case,$e 1=4.4630 e+08 ; n 1=0.4443 ; \quad r 1=1070 ;$

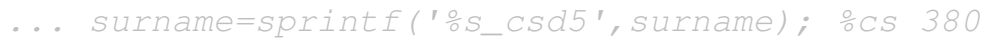

동 $\quad$ case,$e 1=8.0220 e+06 ; n 1=0.4994 ; \quad r 1=1070 ;$

..surname=sprintf ('\%s_csd6', surname); \%cs 50

$\frac{\circ}{\circ}$ end;

\% switch cpu (), ovarying in connective tissue cs

․ case $1, e 2=1.2839 e+06 ; n 3=0.4999 ; r 2=1200 ;$

...surname=sprintf ('\%s_csc1', surname); \%cs 20

동 $2, e 2=3.2025 e+07 ; n 3=0.4965 ; r 2=1200 ;$

...surname=sprintf('\%s_csc2', surname); \%cs 100

case $3, e 2=1.2718 e+08 ; n 3=0.4857 ; r 2=1200 ;$

...surname=sprintf ('\%s_csc3', surname); \%cs 200

case $4, e 2=282480000 ; \quad n 3=0.4667 ; \quad r 2=1200 ;$

..surname=sprintf ("o﹎csc4", surname); \%cs 300

동 $\quad$ case,$e 2=4.4630 e+08 ; n 3=0.4443 ; r 2=1200 ;$

... surname=sprinte ('으C. $5^{\prime}$ ', surname); C.S 380 
70

71

72

73

74

75

76

77

78

79

80

81

82

83

84

85

86

87

88

89

90

91

92

93

94

95

96

97

98

99

100

$101 \mathrm{~d} 1=2 \mathrm{e} 4$;

\& end

$\frac{6}{2}$ end;

$\div$ end;

응 switch cpu (), oVarying in dermal tissue cp

응 case 1, eI=282480000; $n I=0.4667 ; \quad r 1=1070$;

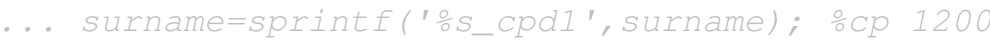

응 case 2, $e 1=2.8427 e+08 ; n 1=0.4759 ; r 1=1070$;

...surname=sprintf('ㅇ﹎cpd2', surname); \%cp 1400

case $3, e 1=284887500 ; \quad n I=0.4792 ; \quad r 1=1070$;

...surname=sprintf('으cod3', surname); ㄷp 1500

응 case 4, e $1=2.8539 e+08 ; n 1=0.4818 ; r 1=1070 ;$

...surname=sprintf ('ㅇs_cpd4', surname); co 1600

동 $\quad$ case,$e 1=2.8615 e+08 ; n 1=0.4857 ; \quad r 1=1070 ;$

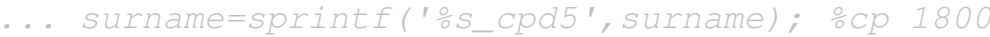

\% switch cpu (), ovarying in connective tissue cp

동 $1, \quad e 2=282480000 ; \quad n 3=0.4667 ; \quad r 2=1200$;

..surname=sprintf ('os_cpc1', surname); \%cp 1200

ase $2, e 2=2.8427 e+08 ; n 3=0.4759 ; r 2=1200$

; ...surname=sprinte ('ㅇs_cpc2', surname); \%cp 1400

case 3, e2=284887500; $n 3=0.4792 ; r 2=1200$;

..surname=sprintf ('⿳亠口口os_cpc3', surname); \%cp 1500

동 $4, e 2=2.8539 e+08 ; n 3=0.4818 ; r 2=1200 ;$

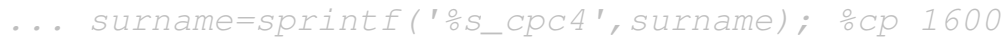

ase $5, \quad e 2=2.8615 e+08 ; n 3=0.4857 ; r 2=1200 ;$

...surname=sprintf ('\%s_cpc5', surname); \%cp 1800

$\div e I=282480000 ; n I=0.4667 ; \quad I=1070 ;$

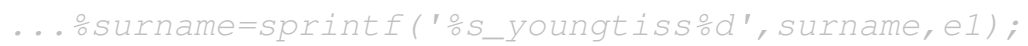

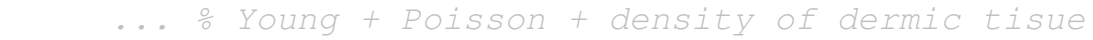

$\frac{\circ}{0} 2=50 e 6 ; n 3=0.49 ; \quad r 2=1200 ;$

00 ... o. Young + Poisson + density of connectic tisue

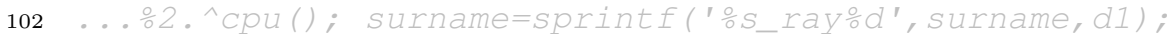


103

104

105

106

107

108

109

110

111

112

114

115

116

117

118

119

120

121

122

123

124

125

126

127

128

$129 \mathrm{~m} 1=1 ; \mathrm{m} 2=2 ; \mathrm{m} 3=1 ; \mathrm{m} 4=1 ;$

130

131

132

133

134

135

136

$\mathrm{d} 2=\mathrm{d} 1$

응응hoosing tissue defining

1

응 end;

$\div$ end:

31

$\frac{\circ}{\circ} m=2 ; \quad m 2=4 ; \quad m 3=2 ; \quad m 4=2 ;$

음 Rayigh attenuation in connective tissue

e2= e1; n3= n1; r2= r1; o Young + Poisson + density of connectictisue

$\mathrm{Tz}=0.5 \mathrm{e}-3 ; \quad$ odermal tissue thickness

$\mathrm{TC}=0.5 \mathrm{e}-3 ; \quad \frac{\circ}{0}$ connective tissue thickness

$\mathrm{Rt}=1.0 \mathrm{e}-3 ; \quad$ otissue extension

응 switch cpu (), oVarying thickness dermal

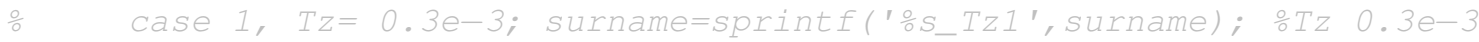

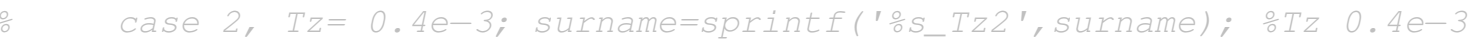

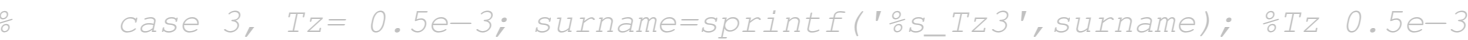

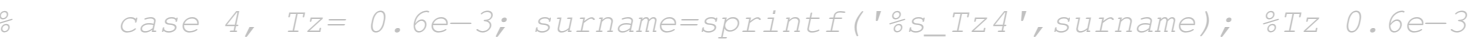

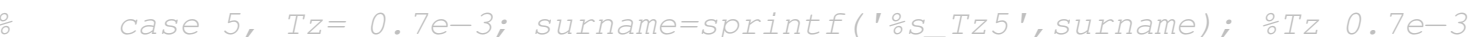

응 sitch cpu (), ovarying thickness connective

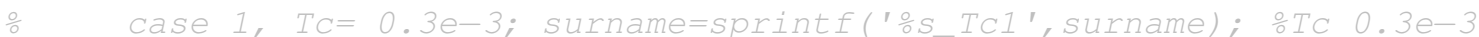

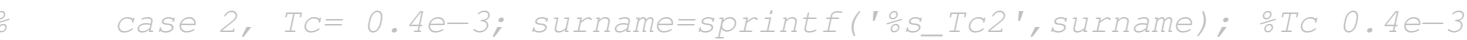

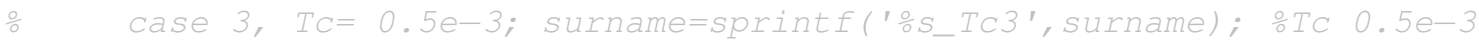

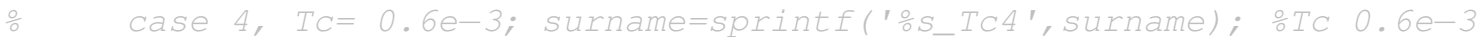

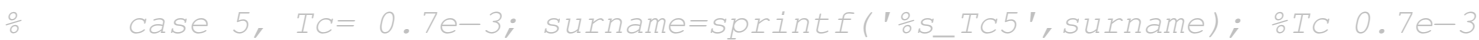

... ․ to be reliable with dt $=25 e-9$ and capture high frequency

$33 \ldots$... to be reliable with dt = 25e-9 and capture high frequency

35 응tch cpu(), 응 Mesh refinement

36 case $0, m 1=1 ; \quad m 2=2 ; m 3=1 ; \quad m 4=1 ; \quad$ surname=[surname, ' $]$; 
146

149

150

151

152

153

154

155

156

157

158

160

162

163

164

165

166

167

168

169

170
응 case 2, $m 1=4 ; m 2=2 ; m 3=1 ; m 4=1 ;$

응 case $3, \quad m I=1 ; \quad m 2=4 ; m 3=1 ; \quad m 4=1 ;$

응 case $4, m 1=1 ; m 2=8 ; m 3=1 ; m 4=1$;

응 case $5, m I=1 ; m 2=2 ; m 3=2 ; m 4=1$;

응 case $6, m I=1 ; \quad m 2=2 ; m 3=4 ; m 4=1$;

응 case $7, m 1=1 ; m 2=2 ; m 3=1 ; \quad m 4=2$;

응 case $8, m I=1 ; \quad m 2=2 ; \quad m 3=1 ; \quad m 4=4 ;$

응

응응 calculate the stiffness matrix and pass it on to FEAP down by

응응 parameters

$a=m o d e l(1) * 1 e-3 ; \quad \frac{\circ}{0}$ piezo width design

$\mathrm{b}=\operatorname{model}(2) \star 1 e-3 ; \quad \div$ piezo thickness design

$\mathrm{pz}=\operatorname{model}(3) \star 1 e-3$

응 piength design

$\mathrm{dd}=\operatorname{model}(4) \star 1 e-3 / 2 ; \quad \frac{0}{0}$ distance sensor piezo internal design

$\mathrm{ds}=\operatorname{model}(5) * 1 \mathrm{e}-3 / 2 ; \quad \frac{\circ}{0}$ means of the external radius of sensor design

$\mathrm{dR}=\operatorname{model}(6) * 1 \mathrm{e}-3 / 2 ; \quad \frac{\circ}{\mathrm{a}}$ inner radius of the sensor design

e =model ( 7$) * 1 e-3 ; \quad \frac{\circ}{\circ}$ external sensor design thickness

Lz $=$ model $(8) \star 1 e-3$;

응 ceramic thickness design surname $=[$ surname, '_2refdisc'] ;

surname $=[$ surname, '_trefdisc'];

surname $=[$ surname, '_2refhdisc'] ;

Iname $=$ [surname, '_2refhpiezo'];

Iname $=$ [surname, '_trefhpiezo'];

surname $=$ [surname, '_2reftissue' ] ;

surname $=$ [surname, '_4reftissue' ]; 
171

172

173

174

175

176

177

178

179

180

181

182

183

184

185

186

187

188

189

190

191

19

193

19

19

19

19

19

19

20

20

202

203

204

case 9, $b=($ model $(2)+0.5) * 1 e-3 ;$ surname='_espesor3' 응 piezo 3

thickness

응 case 10, $b=($ model $(2)+1) * 1 e-3 ;$ surname='_espesor4' $\frac{\circ}{0}$ piezo 4 thickness

$\frac{\circ}{\circ} \frac{\circ}{\circ} d ;$

응 switch cpu(), 응 choose material

응 case 11, $p z=(\operatorname{model}(3)-1.5) * 1 e-3 ;$ surname='_longitudi'

... $\frac{\circ}{0}$ piezo 1 length

응 case $12, p z=(\operatorname{mode} 1(3)-1) * 1 e-3 ;$ surname='_longitud2'

... $\frac{\circ}{0}$ piezo 2 length

응 case 13, pz=model (3)*1e-3; surname='_longitudref'

... 응 piezo ref length

응 case $14, p z=(\operatorname{mode} I(3)+1) * 1 e-3 ;$ surname='_Iongitud3'

.. 응 piezo 3 length

응 case 15, $p z=(\operatorname{model}(3)+3) * 1 e-3 ;$ surname $=$ '_longitud4'

... $\frac{\circ}{\circ}$ piezo 4 length

응 $\quad$ d;

응 switch cpu(), 응 choose material

응 $16, d d=(\operatorname{mode} 1(4) / 2-1) * 1 e-3 ;$ surname='_distanciail'

.. $\frac{\circ}{0}$ internal distance 1 piezo

- case 17, $d d=(\operatorname{model}(4) / 2-0.5) * 1 e-3 ;$ surname='_distanciai2'

.. o internal distance 2 piezo

92 응 $18, d d=$ model (4)*1e-3/2; surname='_distanciairef'

93 ... internal distance ref piezo

194 case 19, $d d=(\operatorname{mode} 1(4) / 2+0.5) * 1 e-3 ;$ surname='_distanciai3'

$195 \ldots$... internal distance 3 piezo

96 ase 20, $d d=(\operatorname{model}(4) / 2+1) * 1 e-3 ;$ surname='_distanciai4'

$197 \ldots$... internal distance 4 piezo

$198 \div \frac{\circ}{0} \in \operatorname{di} ;$

199 응 switch cpul(), 产 choose material

$200 \div$ case $21, d s=(\operatorname{mode} 1(5) / 2-1) * 1 e-3 ;$ surname=' radioext 1 '

201 ... means of the external radius 1

$02 \div$ case 22, $d s=(\operatorname{mode} 1(5) / 2-0.5) * 1 e-3 ;$ surname='_radioext2'

03 ... means of the external radius 2

04 case 23, ds=model (5)*1e-3/2; surname=' radioextref' 


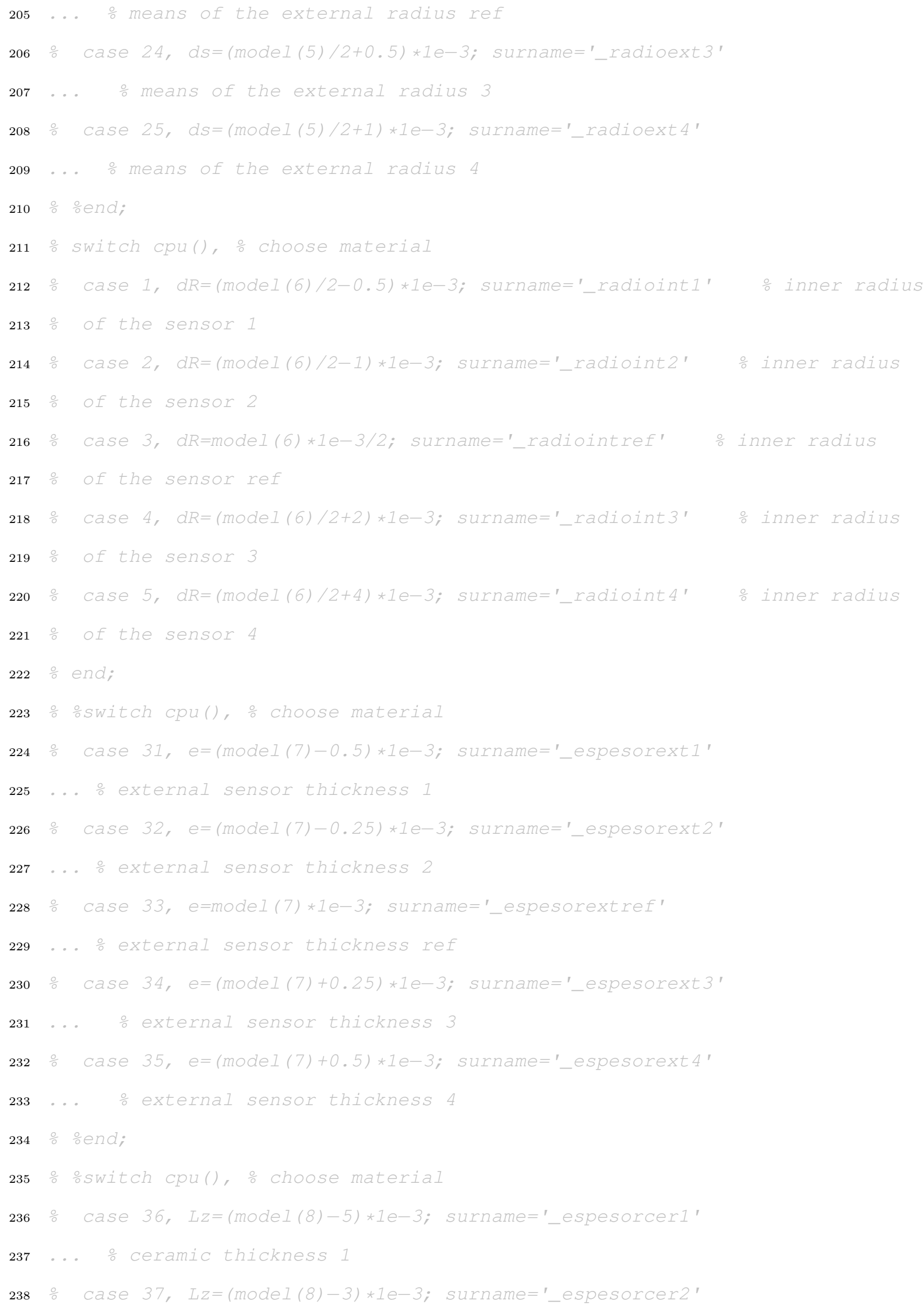




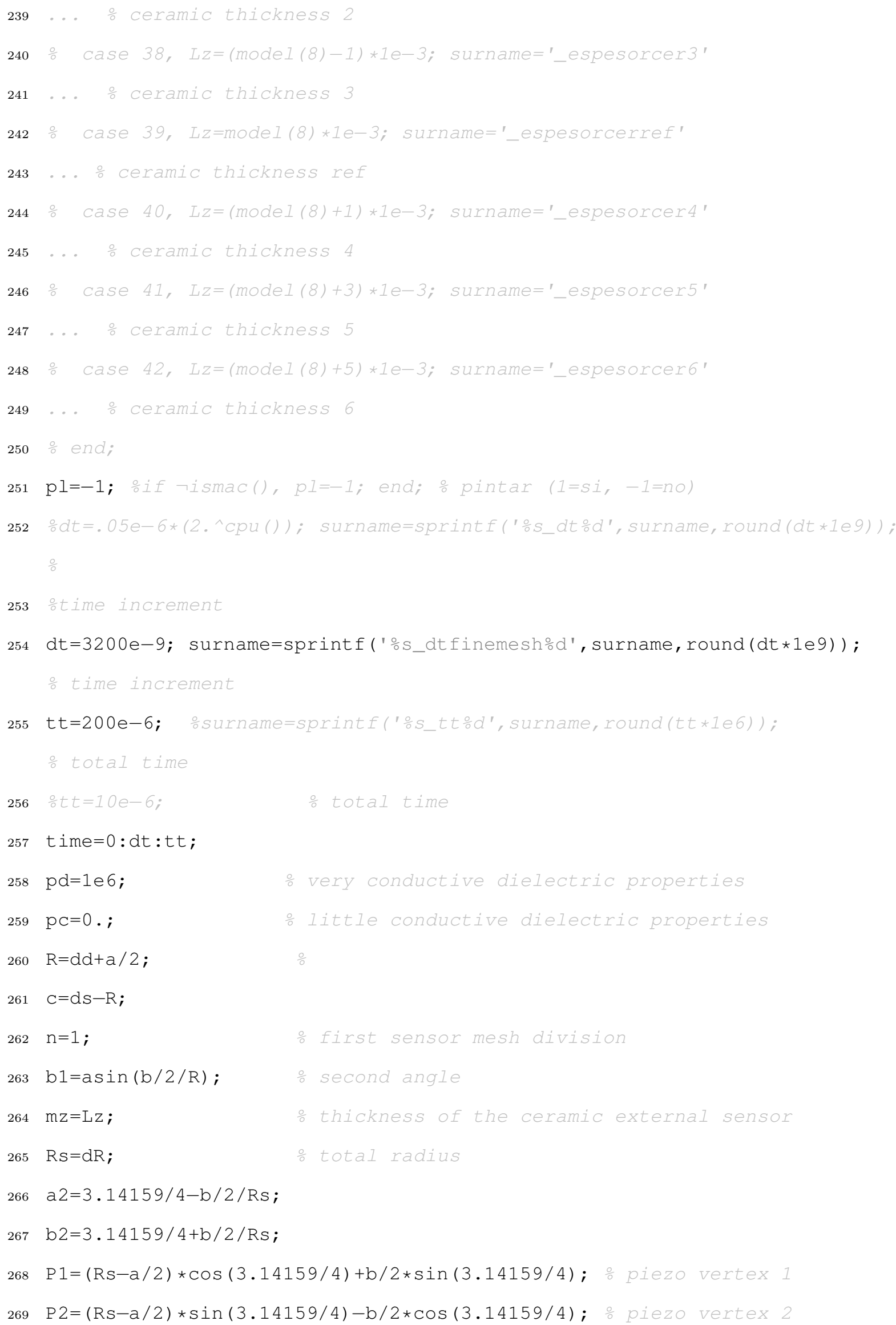


270

271

272

273

274

275

276

277

278

279

280

281

282

283

284

285

286

287

288

289

290

291

292

293

294

295

296

297

298

299

300

301

302

303

$\mathrm{P} 4=(\mathrm{Rs}+\mathrm{a} / 2) \star \sin (3.14159 / 4)-\mathrm{b} / 2 * \cos (3.14159 / 4) ;$ o piezo vertex 4

$\mathrm{P} 5=(\mathrm{Rs}+\mathrm{a} / 2) \star \cos (3.14159 / 4)-\mathrm{b} / 2 * \sin (3.14159 / 4) ;$ o piezo vertex 5

$\mathrm{P} 6=(\mathrm{Rs}+\mathrm{a} / 2) \star \sin (3.14159 / 4)+\mathrm{b} / 2 \star \cos (3.14159 / 4) ;$ o piezo vertex 6

$\mathrm{P} 7=(\mathrm{Rs}-\mathrm{a} / 2) * \cos (3.14159 / 4)-\mathrm{b} / 2 * \sin (3.14159 / 4) ;$ o piezo vertex 7

$\mathrm{P} 8=(\mathrm{Rs}-\mathrm{a} / 2) \star \sin (3.14159 / 4)+\mathrm{b} / 2 * \cos (3.14159 / 4) ;$ o piezo vertex 8

을 $=[.2 e 42 e 4] ;$

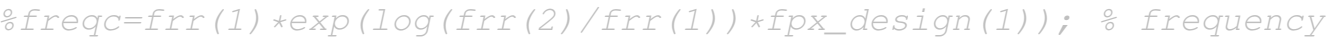

obandwidth=freqc*fpx_design (2);

$\frac{0}{0} \sin =[.005 .02] ;$

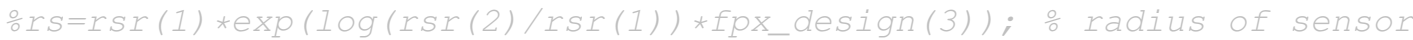

$\frac{0}{0}=i \operatorname{sgen}($ freqc, bandwidth, time) ;

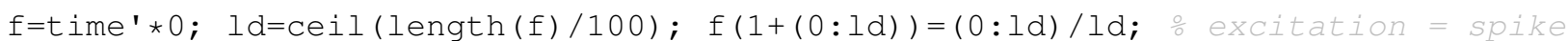

$\mathrm{ft}=\left[\begin{array}{ll}\mathrm{fime} & \mathrm{f}\end{array}\right]$;

save('-ascii', 'forcef', 'ft') ;

fidl=fopen ('param', 'W');

fprintf (fidl, 'PARAmeters \n');

fprintf (fid1, ' $a=013.9 \mathrm{~g} \backslash \mathrm{n}^{\prime}$, $\mathrm{a}$ ) ;

fprintf (fidl, 'b =o $13.9 \mathrm{~g} \backslash \mathrm{n}$ ', b ) ;

fprintf (fidl, ' $d R=\frac{\circ}{1} 3.9 \mathrm{~g} \backslash \mathrm{n}^{\prime}, \mathrm{dR}$ ) ;

fprintf (fidl, ' ds=o13.9g \n', ds) ;

fprintf (fid1, 'dd=o13.9g \n', dd) ;

fprintf (fidl, ' d1=\%13.9g \n', d1);

fprintf (fid1, 'd2=⒔9g \n', d2) ;

fprintf (fid1, 'Lz=⒔9g \n', Lz) ;

fprintf(fid1, 'e =o13.9g \n', e );

fprintf(fid1, 'pz=o13.9g \n',pz);

fprintf (fidl, 'dt=o13.9g \n', dt) ;

fprintf (fidl, 'tt=o⒔9g \n', tt) ;

fprintf(fidl, 'pl=o13.9g \n',pl);

fprintf (fid1, 'Tz=⒔9g \n', Tz);

fprintf (fid1, 'TC=\%13.9g \n', Tc) ; 
fprintf (fid1, 'Rt=\%13.9g \n', Rt) ;

fprintf (fidl, 'R=⒔9g \n', R) ;

fprintf (fidl, 'c=⒔9g \n', c) ;

fprintf (fidl, ' $\mathrm{n}=\frac{0}{1} 13.9 \mathrm{~g} \backslash \mathrm{n}$ ', $\mathrm{n}$ ) ;

fprintf (fid1, 'n2=⒔9g \n', $1 * m 1$ );

fprintf (fidl, 'm=\%13.9g \n', 1) ;

fprintf (fid1, ' $1=\% 13.9 \mathrm{~g} \backslash \mathrm{n}^{\prime}, 3 * \mathrm{~m} 2$ ) ;

fprintf (fid1, ' $11=\% 13.9 \mathrm{~g} \backslash \mathrm{n}^{\prime}, 3 * \mathrm{~m} 4$ ) ;

fprintf (fid1, ' $12=013.9 \mathrm{~g} \backslash \mathrm{n}^{\prime}, 3 \star \mathrm{m} 4$ ) ;

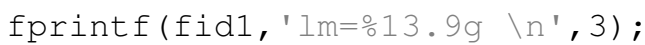

fprintf (fidl, 'o=⒔9g \n', $2 * n$ ) ;

fprintf (fid1, ' $q 1=\circ 13.9 \mathrm{~g} \backslash \mathrm{n}$ ', 1);

fprintf (fid1, ' $q 2=\frac{\circ}{1} 13.9 \mathrm{~g} \backslash \mathrm{n}^{\prime}, 1$ ) ;

fprintf (fid1, ' $q 3=\% 13.9 \mathrm{~g} \backslash \mathrm{n}^{\prime}, 2 \star \mathrm{m} 1$ ) ;

fprintf (fidl, ' $\mathrm{q} 4=\% 13.9 \mathrm{~g} \backslash \mathrm{n}$ ', $1 * \mathrm{~m} 4$ ) ;

fprintf (fid1, ' $q 5=\frac{\circ}{13} .9 \mathrm{~g} \backslash \mathrm{n}^{\prime}$, $4 \star m 4$ ) ;

fprintf (fid1, ' $q 6=\% 13.9 \mathrm{~g} \backslash \mathrm{n}^{\prime}, 2$ ) ;

fprintf ( fid1, 'p=⒔9g \n', $2 * m 3$ );

fprintf (fid1, 'al=o13.9g \n', 3.14159/4);

fprintf (fid1, 'b1=\%13.9g \n', b1);

fprintf (fidl, 'mz=o $\left.13.9 \mathrm{~g} \backslash \mathrm{n}^{\prime}, \mathrm{Lz}\right)$;

fprintf(fidl, 'u=\%13.9g \n',100);

fprintf (fidl, 'Rs=⒔9g \n', Rs) ;

fprintf (fid1, 'a2=⒔9g \n', a2) ;

fprintf (fid1, 'b2=⒔9g \n', b2) ;

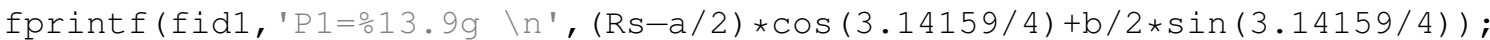

fprintf(fid1, 'P2=⒔9g $\backslash \mathrm{n}^{\prime}$, $\left.(\mathrm{Rs}-\mathrm{a} / 2) \star \sin (3.14159 / 4)-\mathrm{b} / 2 * \cos (3.14159 / 4)\right)$;

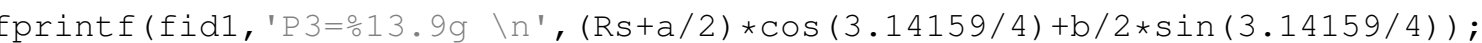

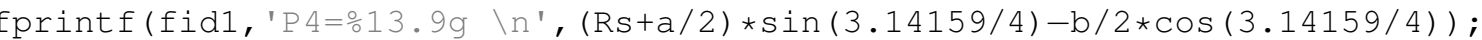

fprintf(fid1,' $\left.\mathrm{P} 5=\frac{\circ}{13} .9 \mathrm{~g} \backslash \mathrm{n}^{\prime},(\mathrm{Rs}+\mathrm{a} / 2) \star \cos (3.14159 / 4)-\mathrm{b} / 2 \star \mathrm{sin}(3.14159 / 4)\right)$;

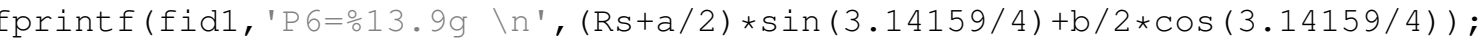

fprintf(fid1,' $\left.P 7=013.9 \mathrm{~g} \backslash \mathrm{n}^{\prime},(\mathrm{Rs}-\mathrm{a} / 2) \star \cos (3.14159 / 4)-\mathrm{b} / 2 \star \mathrm{sin}(3.14159 / 4)\right)$;

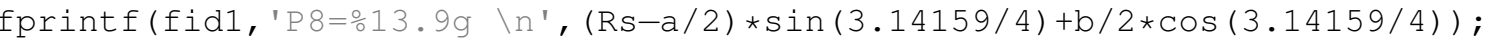

fprintf (fidl, '\n') ; 
339

340

341

342

343

344

345

346

347

348

349

350

351

352

353

354

355

356

357

358

359

360

36

362

363

36

365

366

367

368

369

370

371

fclose(fid1);

fidl=fopen ('materials', 'W');

․ write blocks for FEAP

fprintf(fidl, 'MATErial $1 \backslash \mathrm{n}^{\prime}$ );

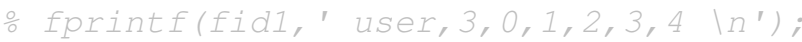

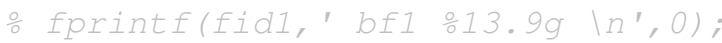

응 fprintf(fid1, ' bf2 ㄷ‥9g $\left.\mid n^{\prime}, 0\right)$;

응 fpintf(fid1, 'bf3ㄴ. $\left.3.99 \backslash n^{\prime}, 0\right)$;

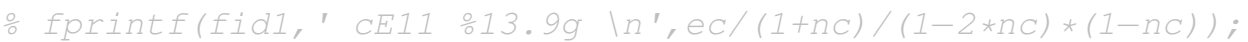

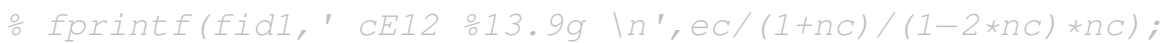

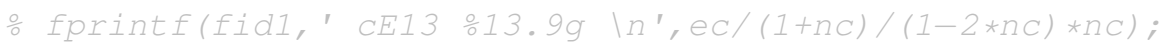

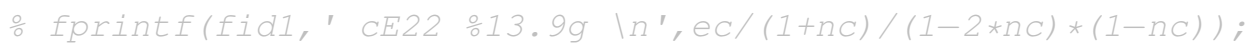

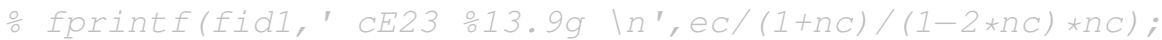

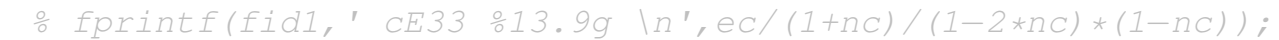

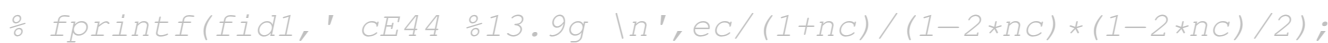

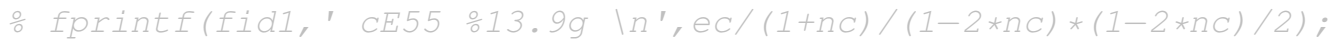

응 fpintf(fid1, 'cE66 1 3.9g In', ec/ (1+nc)/(1-2*nc)*(1-2*nc)/2);

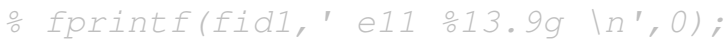

응 fprintf(fid1, ' e12⒔9g $\left.\mid n^{\prime}, 0\right)$;

응 fprintf(fid1, ' e13⒔9g \n', 0);

응 fprintf(fid1, ' e14 ㄷ․ $\left.9 \mathrm{~g} \backslash \mathrm{n}^{\prime}, 0\right)$;

응 fprintf(fidl, " e15 ⒔9g \n', 0);

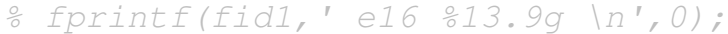

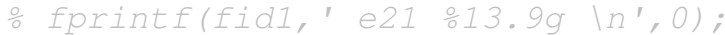

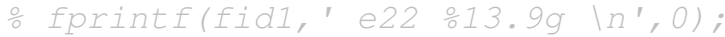

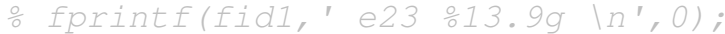

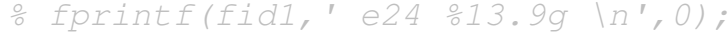

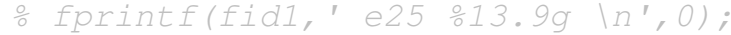

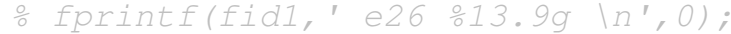

응 fprintf(fid1, ' e $\left.31 \div 13.99 \backslash n^{\prime}, 0\right)$;

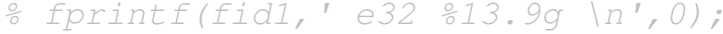

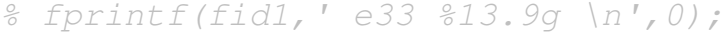

응 fprintf(fidI, ' e $34 \div 13.99$ ।n', 0 );

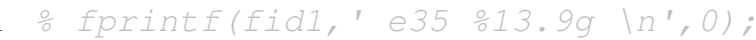


402

403

404 405

2

응 fpintf(fidl, ' e36 ․ $\left.3.9 \mathrm{~g} \backslash \mathrm{n}^{\prime}, 0\right)$;

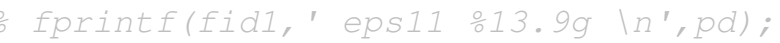

응 fprintf(fid1, ' eps12 ⒔9g In', 0);

응 fprintf(fid1, ' eps13⒔9g In', o);

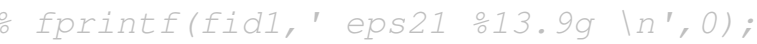

응 fprintf(fid1, ' eps22 ⒔9g ln',pd);

응 fprintf(fid1, ' eps23⒔9g $\ln ^{\prime}, 0$ );

응 fprintf(fid1, ' eps31 으. 99 In', 0);

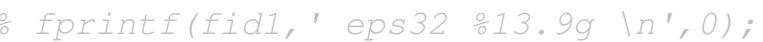

응 fprintf(fidl, ' eps33⒔9g (n', pd);

응 fpintf(fidl, ' rho ․ $\left.3.9 \mathrm{~g} \backslash \mathrm{n}^{\prime}, \mathrm{rc}\right)$;

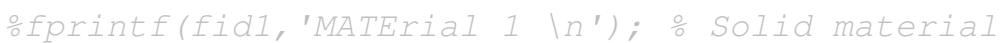

fprintf(fidl, ' SOLID $\backslash \mathrm{n}^{\prime}$ ) ;

fprintf(fid1,' ELAStic ISOTropic \%13.9g \%13.9g \n', ec, nc);

fprintf(fid1,' density mass \%13.9g \n', rc);

fprintf(fid1, ' damping rayleigh \%13.9g \%13.9g \n', dc,0);

fprintf (fidl, '\n');

fprintf(fidl, 'MATErial $4 \backslash \mathrm{n}^{\prime}$ ); $\frac{\circ}{0}$ Demic tissue

fprintf(fidl, ' SOLID $\left.\backslash n^{\prime}\right)$;

fprintf(fid1,' ELAStic ISOTropic \%13.9g \%13.9g \n', e1, n1);

fprintf(fid1,' density mass ⒔9g \n', r1);

fprintf(fid1, ' damping rayleigh $\frac{0}{13.9 \mathrm{~g}} \% 13.9 \mathrm{~g} \backslash \mathrm{n}$ ', d1, 0 );

fprintf (fidl, '\n');

fprintf(fid1, 'MATErial $5 \backslash \mathrm{n}$ '); 응 connective tissue

fprintf (fidl, ' SOLID \n');

fprintf(fid1, ' ELAStic ISOTropic \%13.9g \%13.9g \n', e2, n3);

fprintf(fid1, ' density mass 1‥9g \n', r2);

fprintf(fid1, ' damping rayleigh $\% 13.9 \mathrm{~g} \div 13.9 \mathrm{~g} \backslash \mathrm{n}$ ', d2, 0 );

fprintf (fidl, '\n');

fclose ( fidl);

oif and (isunix, not (ismac) ), unix ('./dynfeap <y');

if and(isunix, not (ismac)), unix('./feap_mse2 <y'); 


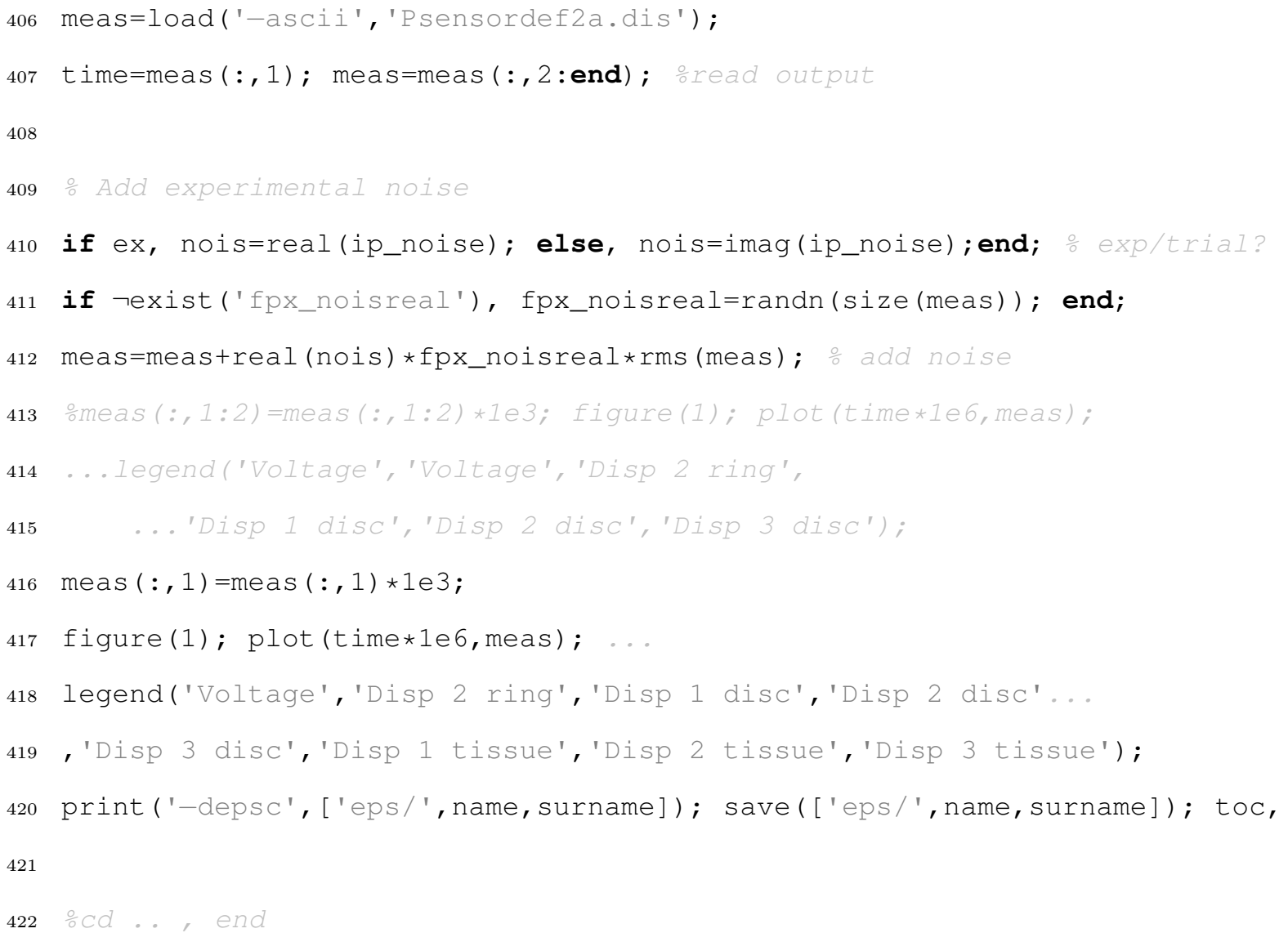


3

4

6

7 action

$8 \mathrm{n} 1 \mathrm{~s}$

9 mainfile

remotedir

1

12

15

16 switch action

17

18

19

22
$2011-03-22$

\section{2-07-10}

mse 2:

-scp-without-password.htmI

$=1: 5 ; \quad$ which cpu:s

$=$ 'fpx_mea_senstor.m';

$=$ 'senstor';

filecontents $=\{$ 'feapname' 'Isensordef2' 'cpu.m' 'isgen.m' 'rms.m'...

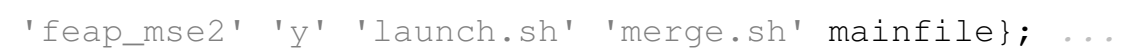

case $1 \%$ send files and launch job

o script to remotely multiply folders from master to cpu??, and run all jobs

fidl=fopen ( 'launch.sh', 'W');

for $n 1=n 1 s ;$ fprintf(fid1, 'rm -r cpuo02d $\left.\backslash n^{\prime}, n 1\right)$; end;

for $n 1=n 1 s$; fprintf(fidl, 'rsync-avu master/ cpu\%02d/ \n', n1); end;

for n1=n1s; fprintf(fid1, 'cd cpuo02d; matbg \%s out.txt ; cd .. \n',n1,mainfile) end; fclose(fidl);

o script to remotely merge from cpu?? to master

fidl=fopen ('merge.sh', 'w');

for $n 1=n 1 s ;$ fprintf(fidl, 'rsync -avu cpuo02d/ master/ \n', n1); end; . fclose (fid1);

\% send local files to remote master folder; remotely run./Iaunch.sh

unix(['rsync -avu - files-from=filelist.txt ogrusemse2.ugr.es: ",remotedir, . . '/master']);

unix(['ssh grusemse2.ugr.es ' 'cd '...

, remotedir, '; chmod 744 master/*.sh; mv master/*.sh . ; ./launch.sh' ' ']);

delete ('filelist.txt',' launch.sh', 'merge.sh'); 
35 case 2 \% retrieve (remotely run./merge.sh; get files from eps/)

36 unix(['ssh grusemse2.ugr.es ' 'cd ',remotedir,' ; ./merge.sh' ' ']);

37 unix(['rsync -avv grusemse2.ugr.es: ', remotedir, '/master/eps/ eps/ ']);

38 end; 
1 ․․․․ Melchor 31-5-2012

2 탐 young modulus and $n$ Poisson coeficent with wave speed determinated

$4 \mathrm{cp}=1500 ; \quad \frac{\circ}{\circ}$ speed of p-wave

$6 \mathrm{CS}=50$; $\frac{\circ}{\circ}$ speed of s-wave

$8 \div \cos =\operatorname{sart}(E *(1-n) /((1+n) *(1-2 * n)) / 1070) ;$

10

11

13

$14 \mathrm{n}=\left(\mathrm{cp} \mathrm{p}^{\wedge} 2 * 1070-2 * \mathrm{cs}^{\wedge} 2 * 1070\right) /\left(2 * \mathrm{cp}^{\wedge} 2 * 1070-2 * \mathrm{cs}^{\wedge} 2 * 1070\right)$

$\div(M-2 * G) /(2 * M-2 * G)$

$16 E=C S^{\wedge} 2 \star 1070 \star\left(3 * C P^{\wedge} 2 * 1070-4 * C S^{\wedge} 2 \star 1070\right) /\left(\mathrm{CP}^{\wedge} 2 \star 1070-\mathrm{CS}^{\wedge} 2 \star 1070\right) ; \div G(3 M-4 G) /(M-G)$

17

18

19

20

$21 \div E=e c ; n=n c ; r=r c ;$

$22 \div c p=\operatorname{sqrt}(E *(1-n) /((1+n) *(1-2 * n)) / r)$

$23 \div \operatorname{cs}=\operatorname{sort}(E / 2 *(1+n) / r)$ 


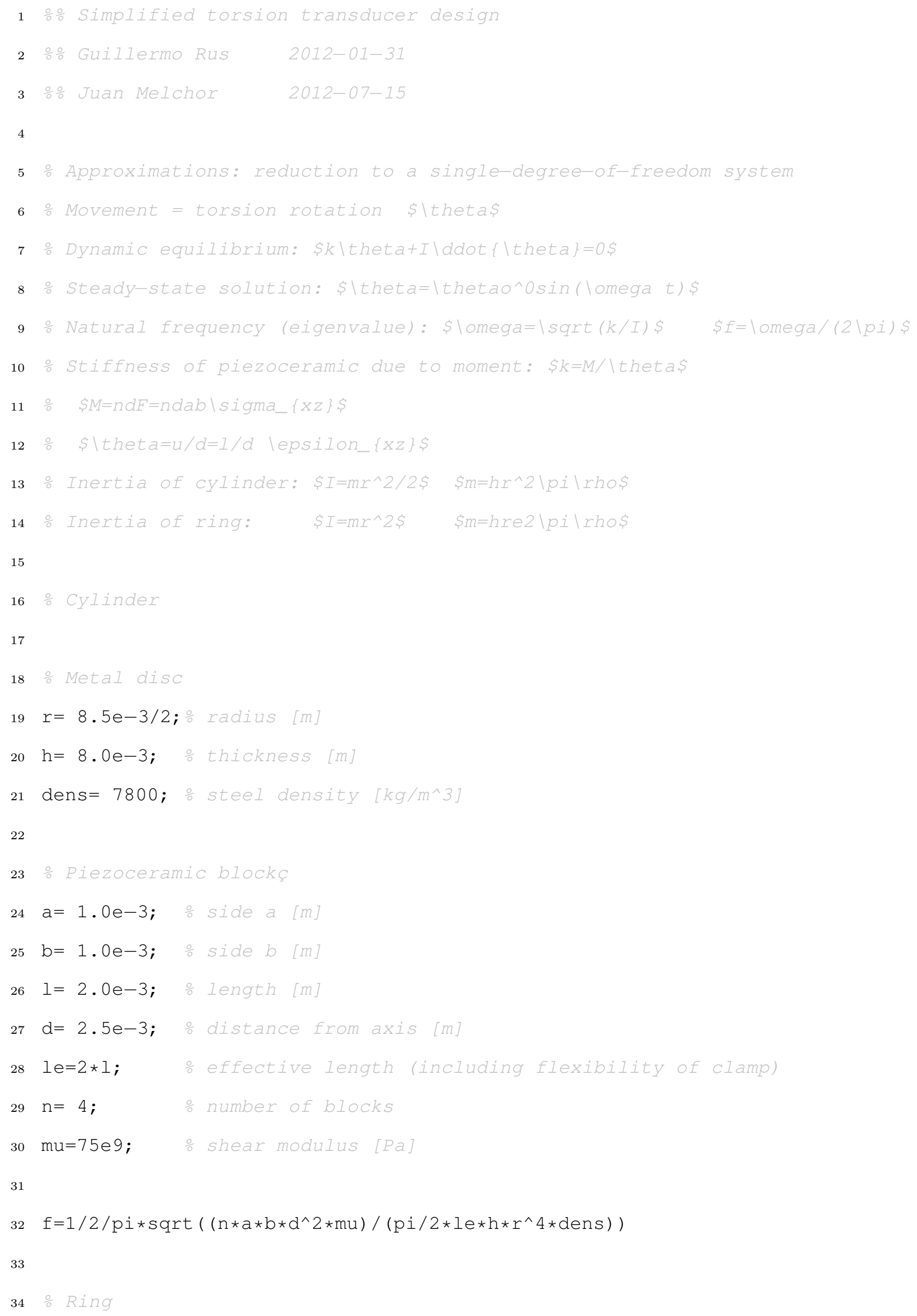


50

$51 \mathrm{f}=1 / 2 / \mathrm{pi} * \operatorname{sqrt}\left(\left(\mathrm{n} * \mathrm{a} * \mathrm{~b} * \mathrm{~d}^{\wedge} 2 * \mathrm{mu}\right) /\left(2 * \mathrm{pi} * 1 \mathrm{e} * \mathrm{~h} * \mathrm{e} * \mathrm{r}^{\wedge} 3 * \mathrm{dens}\right)\right)$ 


\section{List of Figures}

2.1 Spike function . . . . . . . . . . . . . . . . . . 9

2.2 FEM for Sensor in Model A . . . . . . . . . . . . . 13

2.3 Sensor A: Valpey Fischer fabrication . . . . . . . . . . . 14

2.4 The geometry of the transducer . . . . . . . . . . 17

2.5 example of measurement validating simple design . . . . . 20

2.6 Time step convergence . . . . . . . . . . . . . . . . . 21

2.7 High incremental time time step convergence . . . . . . . . 21

2.8 The geometry of the mesh . . . . . . . . . . . . 22

2.9 Mesh convergence . . . . . . . . . . . . . . . 24

2.10 Sensitivity width piezo total time 200 radial displacement disc and voltage signal . . . . . . . . . . . 25 
2.11 Sensitivity width piezo total time 200 turn displacement disc and radial displacement ring . . . . . . . . . . . 26

2.12 Sensitivity thickness piezo total time 200 radial displacement disc and voltage signal $\ldots \ldots \ldots \ldots . \ldots 26$

2.13 Sensitivity thickness piezo total time 200 turn displacement disc and radial displacement ring . . . . . . . . . . . . 27

2.14 Sensitivity length piezo total time 200 radial displacement disc and voltage signal . . . . . . . . . . . . . . 27

2.15 Sensitivity length piezo total time 200 turn displacement disc and radial displacement ring . . . . . . . . . . . 28

2.16 Sensitivity inner distance total time 200 radial displacement disc and voltage signal . . . . . . . . . . . . . . 29

2.17 Sensitivity inner distance total time 200 turn displacement disc and radial displacement ring . . . . . . . . . . . . 29

2.18 Sensitivity outer radius total time 200 radial displacement disc and voltage signal . . . . . . . . . . . . 30

2.19 Sensitivity length outer radius time 200 turn displacement disc and radial displacement ring . . . . . . . . . . . 30

2.20 Sensitivity inner radius total time 200 radial displacement disc and voltage signal . . . . . . . . . . . . . 31 
2.21 Sensitivity length inner radius time 200 turn displacement disc and radial displacement ring . . . . . . . . . . . . . 31

2.22 Sensitivity thickness outer sensor total time 200 radial displacement disc and voltage signal . . . . . . . . . . 32

2.23 Sensitivity thickness outer sensor total time 200 turn displacement disc and radial displacement ring . . . . . . . . 32

2.24 Sensitivity steel thickness total time 200 radial displacement disc and voltage signal . . . . . . . . . . . . . 33

2.25 Sensitivity steel thickness total time 200 turn displacement disc and radial displacement ring . . . . . . . . . . 33

2.26 Sensitivity of dermic tissue total time 200 turn radial displacement disc and voltage signal varying second wave speed

2.27 Sensitivity of dermic tissue total time 200 turn displacement disc and radial displacement ring varying second wave speed

2.28 Sensitivity of connective tissue total time 200 turn radial displacement disc and voltage signal varying second wave speed

2.29 Sensitivity of connective tissue total time 200 turn displacement disc and radial displacement ring varying second wave

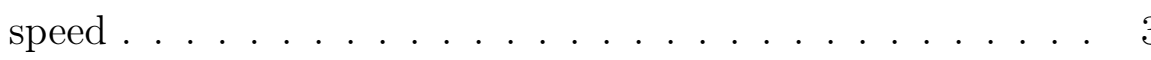

2.30 Sensitivity of dermic tissue total time 200 turn radial displacement disc and voltage signal varying primary wave speed 35 
2.31 Sensitivity of dermic tissue total time 200 turn displacement disc and radial displacement ring varying primary wave speed 36

2.32 Sensitivity of connective tissue total time 200 turn radial displacement disc and voltage signal varying primary wave speed 36

2.33 Sensitivity of connective tissue total time 200 turn displacement disc and radial displacement ring varying primary wave

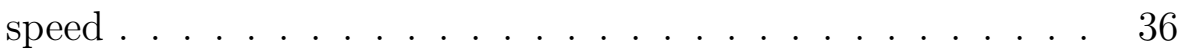

2.34 Sensitivity of dermic tissue thickness total time 200 turn radial displacement disc and voltage signal . . . . . . . . 37

2.35 Sensitivity of dermic tissue thickness total time 200 turn displacement disc and radial displacement ring . . . . . . . 37

2.36 Sensitivity of connective tissue thickness total time 200 turn radial displacement disc and voltage signal . . . . . . . . . 37

2.37 Sensitivity of connective tissue thickness total time 200 turn displacement disc and radial displacement ring . . . . . . . 38

2.38 Different states of torsional transducer . . . . . . . . . . 39

3.1 Simplified system for measuring ultrasound wave distortion through tissue and reconstruct mechanical properties. . . . . 42

3.2 Simulated measurements for model design, noise $0.10 \%$. . . 49 
3.3 Cost functional as a function of the parameters with reference $G_{d}$ and $G_{c} \ldots \ldots \ldots \ldots \ldots \ldots$

3.4 Dependency of the POD on the pathology amount. . . . . . 52

3.5 POD optimization . . . . . . . . . . . 53

A.1 Dynamic 1D problem . . . . . . . . . . . . . 63

A.2 Different states of piezoelectric 3D element . . . . . . . . . 64 


\section{List of Tables}

2.1 Windows characteristics. . . . . . . . . . . . . 12

2.2 Final dimensions of transducers . . . . . . . . . . . 16

2.3 Final dimensions of transducers . . . . . . . . . . . 16

2.4 Validation ........................ . . 20

2.5 Final dimensions of transducers . . . . . . . . . . . 23

2.6 Final dimensions of transducers . . . . . . . . . . . 25

2.7 Mechanical and geometrical properties of the considered ma-

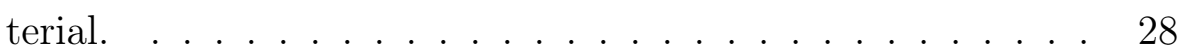

2.8 Mechanical and geometrical properties of the considered ma-

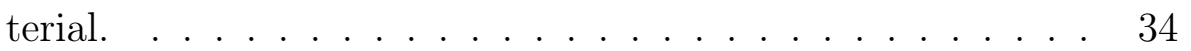

2.9 Mechanical and geometrical properties of the considered material. . . . . . . . . . . . . . . . 38 
2.10 Final dimensions of transducers . . . . . . . . . . . . 38

A.1 Validation 1D Piezo material . . . . . . . . . . . . 65 


\section{Bibliography}

[1] A. Glazounov, Q. Zhang, C. Kim, Torsional actuator based on mechanically amplified shear piezoelectric response, Sensors and Actuators A: Physical 79 (1) (2000) 22-30.

[2] P. Harkness, A. Cardoni, M. Lucas, A comparison of coupling and degenerating mode in longitudinal-torsional step horns, IEEE Transactions on Ultrasonics, Ferroelectrics and Frequency Control .

[3] T. Szabo, Diagnostic ultrasound imaging: inside out, Academic Press, 2004 .

[4] J. Thijssen, W. Verhoef, M. Cloostermans, Optimization of ultrasonic transducers, Ultrasonics 23 (1) (1985) 41-46.

[5] E. Heikkola, M. Laitinen, Model-based optimization of ultrasonic transducers, Ultrasonics sonochemistry 12 (1) (2005) 53-57.

[6] A. Schröder, J. Rautenberg, B. Henning, Evaluation of cost functions for FEA based transducer optimization, Physics Procedia 3 (1) (2010) 1003-1009.

[7] E. Heikkola, K. Miettinen, P. Nieminen, Multiobjective optimization of an ultrasonic transducer using NIMBUS, Ultrasonics 44 (4) (2006) $368-380$. 
[8] B. Fu, T. Hemsel, J. Wallaschek, Piezoelectric transducer design via multiobjective optimization, Ultrasonics 44 (2006) e747-e752.

[9] J. Kim, O. Kwon, Vibration characteristics of piezoelectric torsional transducers, Journal of sound and vibration 264 (2) (2003) 453-473.

[10] S. Kaneko, S. Nomoto, H. Yamamori, K. Ohya, Load characteristics of a bolted Langevin torsional transducer, Ultrasonics 34 (2) (1996) $239-241$.

[11] S. Lin, Study on the Langevin piezoelectric ceramic ultrasonic transducer of longitudinal-flexural composite vibrational mode, Ultrasonics 44 (1) (2006) 109-114.

[12] M. Aoyagi, T. Suzuki, Y. Tomikawa, Characteristics of a bolt-clamped torsional vibrator using shear-mode piezo-ceramics sandwiched in the axial direction, Ultrasonics 34 (2) (1996) 219-222.

[13] T. Harada, N. Ishikawa, T. Kanda, K. Suzumori, Y. Yamada, K. Sotowa, Droplet generation using a torsional Langevin-type transducer and a micropore plate, Sensors and Actuators A: Physical 155 (1) (2009) 168-174.

[14] J. Li, P. Liu, H. Ding, W. Cao, Modeling characterization and optimization design for PZT transducer used in Near Field Acoustic Levitation, Sensors and Actuators A: Physical .

[15] H. Lin, W. Fang, A rib-reinforced micro torsional mirror driven by electrostatic torque generators, Sensors and Actuators A: Physical 105 (1) (2003) 1-9.

[16] H. Kwun, W. Jolly, G. Light, E. Wheeler, Effects of variations in design parameters of ultrasonic transducers on performance characteristics, Ultrasonics 26 (2) (1988) 65-72. 
[17] C. Dutcher, D. Venerus, Compliance effects on the torsional flow of a viscoelastic fluid, Journal of Non-Newtonian Fluid Mechanics 150 (2-3) (2008) 154-161.

[18] F. Wang, X. Zhao, D. Zhang, Y. Wu, Development of novel ultrasonic transducers for microelectronics packaging, Journal of materials processing technology 209 (3) (2009) 1291-1301.

[19] S. Van Kervel, J. Thijssen, A calculation scheme for the optimum design of ultrasonic transducers, Ultrasonics 21 (3) (1983) 134-140.

[20] Y. Sunny, C. Bawiec, A. Nguyen, J. Samuels, M. Weingarten, L. Zubkov, P. Lewin, Optimization of un-tethered, low voltage, 20$100 \mathrm{kHz}$ flexural transducers for biomedical ultrasonics applications, Ultrasonics .

[21] H. Windhagen, H. Bail, A. Schmeling, S. Kolbeck, A. Weiler, M. Raschke, A new device to quantify regenerate torsional stiffness in distraction osteogenesis, Journal of biomechanics 32 (8) (1999) 857860.

[22] R. Taylor, FEAP-ein finite element analysis programm, Ing.Gemeinschaft Klee \& Wrigges, 1987.

[23] G. Rus, R. Palma, J. Pérez-Aparicio, Optimal measurement setup for damage detection in piezoelectric plates, International Journal of Engineering Science 47 (4) (2009) 554-572.

[24] R. Taylor, FEAP manual, 2003.

[25] K. Yonetsu, M. Ohki, S. Kumazawa, S. Eida, M. Sumi, T. Nakamura, Parotid tumors: differentiation of benign and malignant tumors with quantitative sonographic analyses, Ultrasound in Med.\&Biol. 30 (5) (2004) 567-574. 
[26] T. Matsumura, T. Umemoto, Y. Fujihara, E. Ueno, T. Shiina, T. Mitake, Measurement of Elastic Property of Breast Tissue for Elasticity Imaging, in: 2009 IEEE International Ultrasonics Symposium (IUS), Roma, Italy, 2009.

[27] T. Bigelow, B. McFarlin, W. O'Brien Jr., M. Oelze, In vivo ultrasonic attenuation slope estimates for detecting cervical ripening in rats: Preliminary results, Journal of the Acoustical Society of America 123 (3) (2008) 1794-1800, cited By (since 1996) 0.

[28] B. L. McFarlin, W. D. O’brien, Jr., M. L. Oelze, J. F. Zachary, R. White-Traut, Ultrasound insertion loss of the rat cervix, American Journal of Obstetrics and Gynecology 193 (6, Supplement 1) (2005) S154-S154, ISSN 0002-9378, 26th Annual Meeting of the Society for Maternal-Fetal Medicine - The Pregnancy Meeting.

[29] B. McFarlin, W. D. O’brien, M. L. Oelze, J. F. Zachary, R. WhiteTraut, Quantitative ultrasound predicts cervical ripening in the rat, American Journal of Obstetrics and Gynecology 193 (6, Supplement 1) (2005) S153-S153, ISSN 0002-9378, 26th Annual Meeting of the Society for Maternal-Fetal Medicine - The Pregnancy Meeting.

[30] D. Bader, P. Bowker, Mechanical characteristics of skin and underlying tissues in vivo, Biomaterials 4 (4) (1983) 305-308, ISSN 0142-9612.

[31] A. S. Ahuja, Tissue as a Voigt body for propagation of ultrasound, Ultrasonic Imaging 1 (2) (1979) 136-143, ISSN 0161-7346.

[32] J. Pereira, J. Mansour, B. Davis, Dynamic measurement of the viscoelastic properties of skin, Journal of Biomechanics 24 (2) (1991) 157-162, ISSN 0021-9290. 
[33] G. Rus, S. Y. Lee, S. Y. Chang, S. C. Wooh, Optimized damage detection of steel plates from noisy impact test, International Journal for Numerical Methods in Engineering 68 (2006) 707-727.

[34] D. Goldberg, Genetic algorithms in search, optimization and machine learning, Addison-Wesley Publ, Reading, Massachussets, etc., 1989.

[35] R. Gallego, G. Rus, Identification of cracks and cavities using the topological sensitivity boundary integral equation, Computational Mechanics 33 (2004) 154-163.

[36] A. Saltelli, K. Chan, E. M. Scott, Sensitivity Analysis, John Wiley \& Sons LTD, 2000.

[37] L. Rade, B. Westergren, Mathematics Handbook for Science and Engineering, Springer, 1999.

[38] M. D, L. RT, M. T, Long-term medical and social consequences of preterm birth, N Engl J Med. 17 (2008) 262-273.

[39] B. Blondel, A. Macfarlane, M. Gissler, G. Breart, J. Zeitlin, Preterm birth and multiple pregnancy in European countries participating in the PERISTAT project, BJOG 113 (2006) 528-535.

[40] R. L. Goldenberg, J. F. Culhane, J. D. Iams, R. Romero, Epidemiology and causes of preterm birth, The Lancet 371 (9606) (2008) 75-84, ISSN 0140-6736.

[41] I. of Medicine, Preterm Birth: Causes, Consequences, and Prevention, National Academies Press, 500 Fifth Street, N.W., Lockbox 285, Washington, DC 20055. http://www.nap.edu .

[42] P. Delsanto, T. Whitcombe, H. Chaskelis, R. Mignogna, Connection 
machine simulation of ultrasonic wave propagation in materials. I: the one-dimensional case, Wave Motion 16 (1) (1992) 65-80. 